\title{
MERCURY INJECTION CAPILLARY PRESSURE RESULTS FROM OUTCROP SAMPLES IN THE HOMER AREA OF COOK INLET
}

by

\author{
Andrea Loveland ${ }^{1}$
}

\section{INTRODUCTION}

This chapter summarizes mercury-injection capillary pressure data for 12 outcrop samples collected from the Beluga and Sterling formations in the vicinity of Homer, Alaska, during the 2007 field season (figs. 1 and 2). All samples are tied to measured stratigraphic sections, which are presented in LePain and others (this volume). Sample numbers correspond to measured section numbers and the sample position (meters above base of section) in a measured section. For example, sample 07JRM002-3.5a was collected 3.5 meters above the base of measured section 07JRM002. Analyses were performed by PetroTech Associates, Houston, Texas.

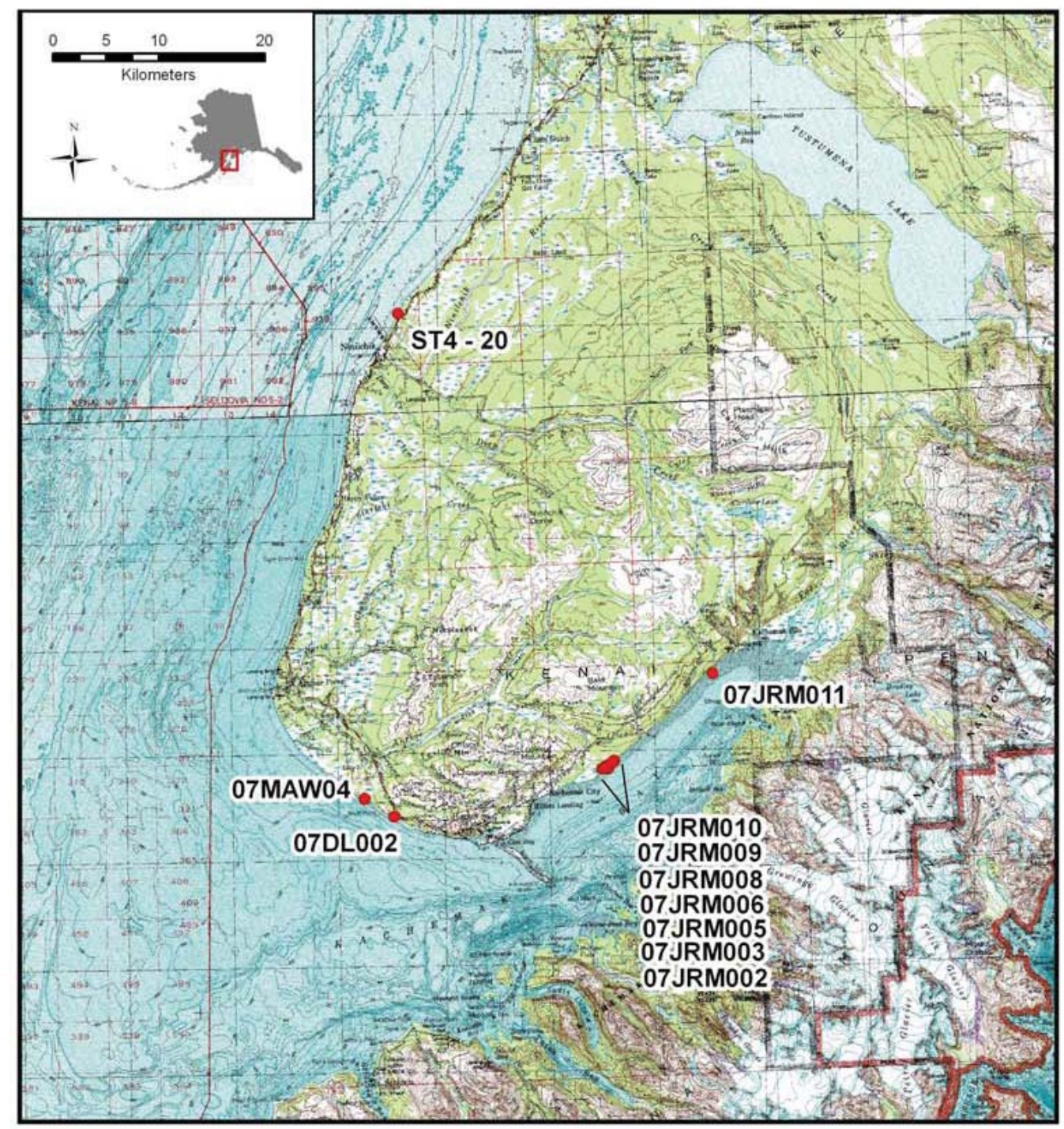

Figure 1. Map showing the Cook Inlet area and the locations of the measured sections from which the MICP samples were collected.

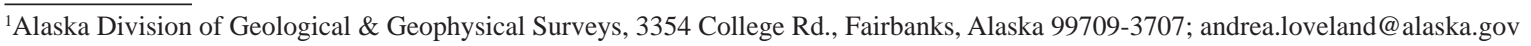


Potential seal capacity data for gas/water and oil/water systems at four different saturation levels are summarized in figures 3a-b and 4a-b, respectively. Figures 5 and 6 illustrate the potential seal capacity for gas and oil, respectively, at $7.5 \%$ seal saturation. Mercury injection capillary pressure and pore aperture curves are presented in figures 7a-20c. Sample collection sites and lithology data are summarized in table 1. The pore system and capillary properties are summarized in table 2 . Oil and gas column heights are summarized in table 3 . Mercury injection capillary pressure data for each sample are presented in tables 4 through 17.

\section{ACKNOWLEDGMENTS}

Funding for this work was provided by Benchmark Oil and Gas, Pioneer Natural Resources, Chevron North American Exploration and Production Company, and the State of Alaska.

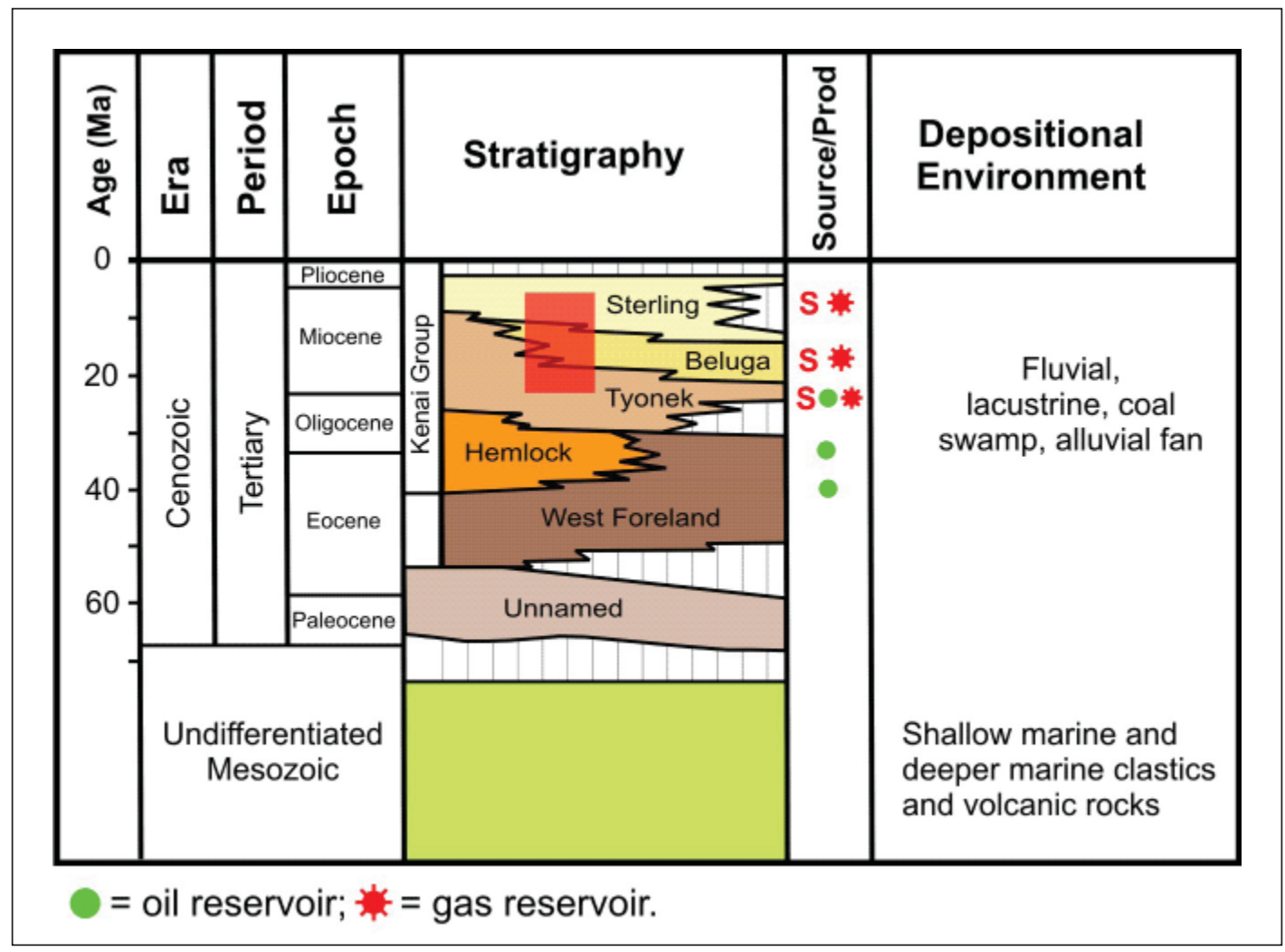

Figure 2. Simplified stratigraphic column showing Tertiary nonmarine formations in Cook Inlet basin. Vertical red bar shows stratigraphic interval where MICP samples were collected. Modified from Swenson (2002). 


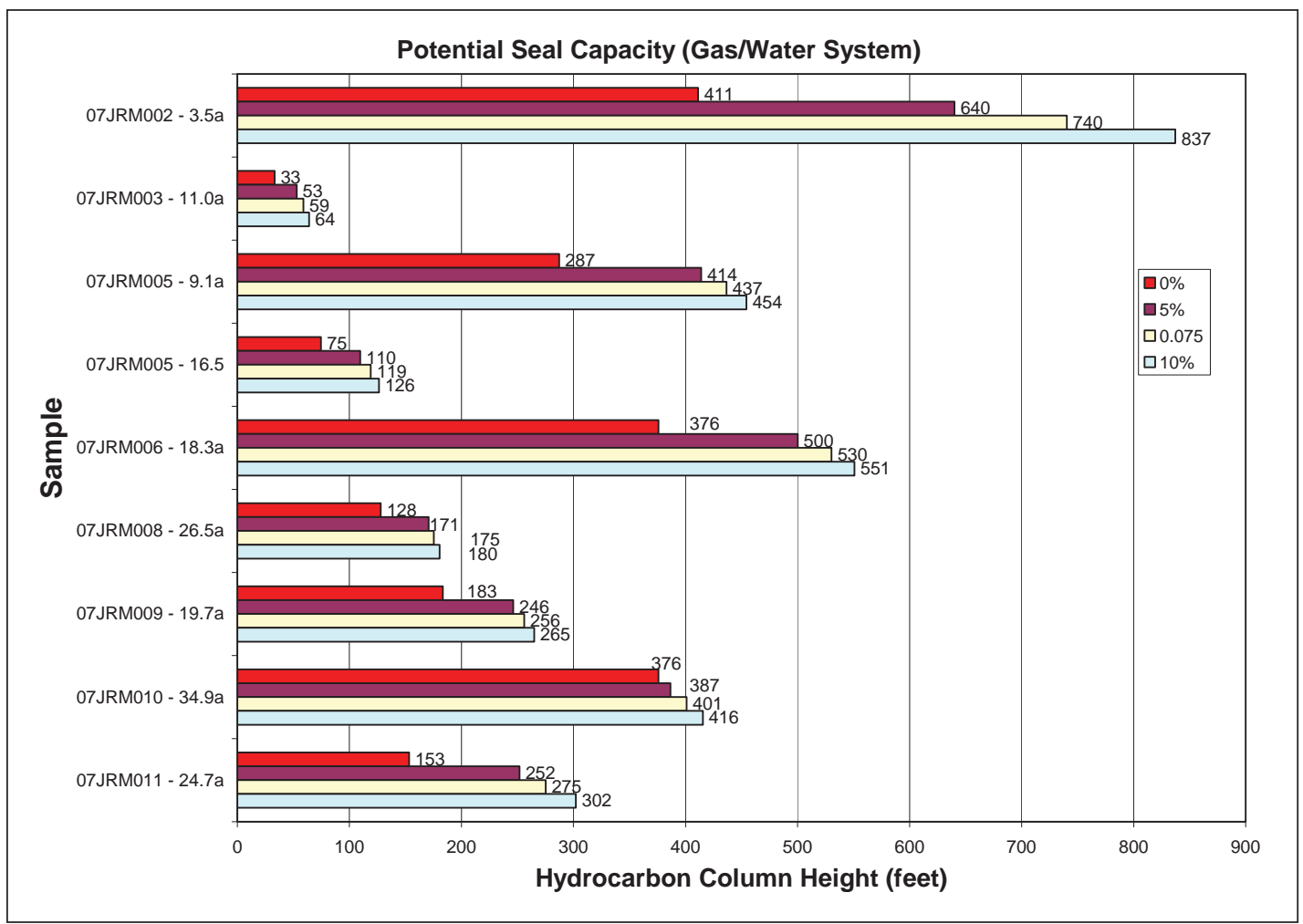

Figure 3a. Potential seal capacity in a gas/water system.

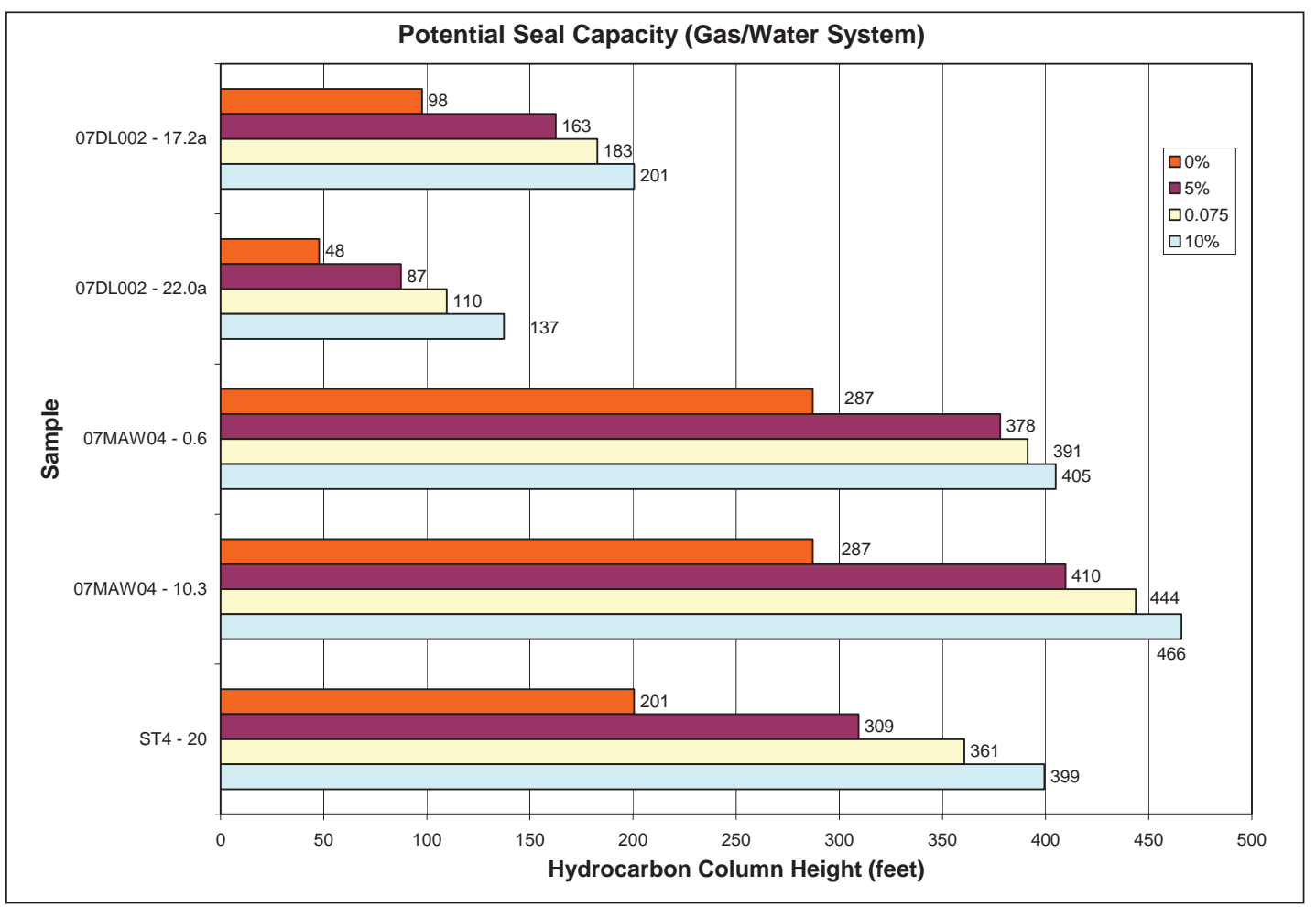

Figure 3b. Potential seal capacity in a gas/water system. 


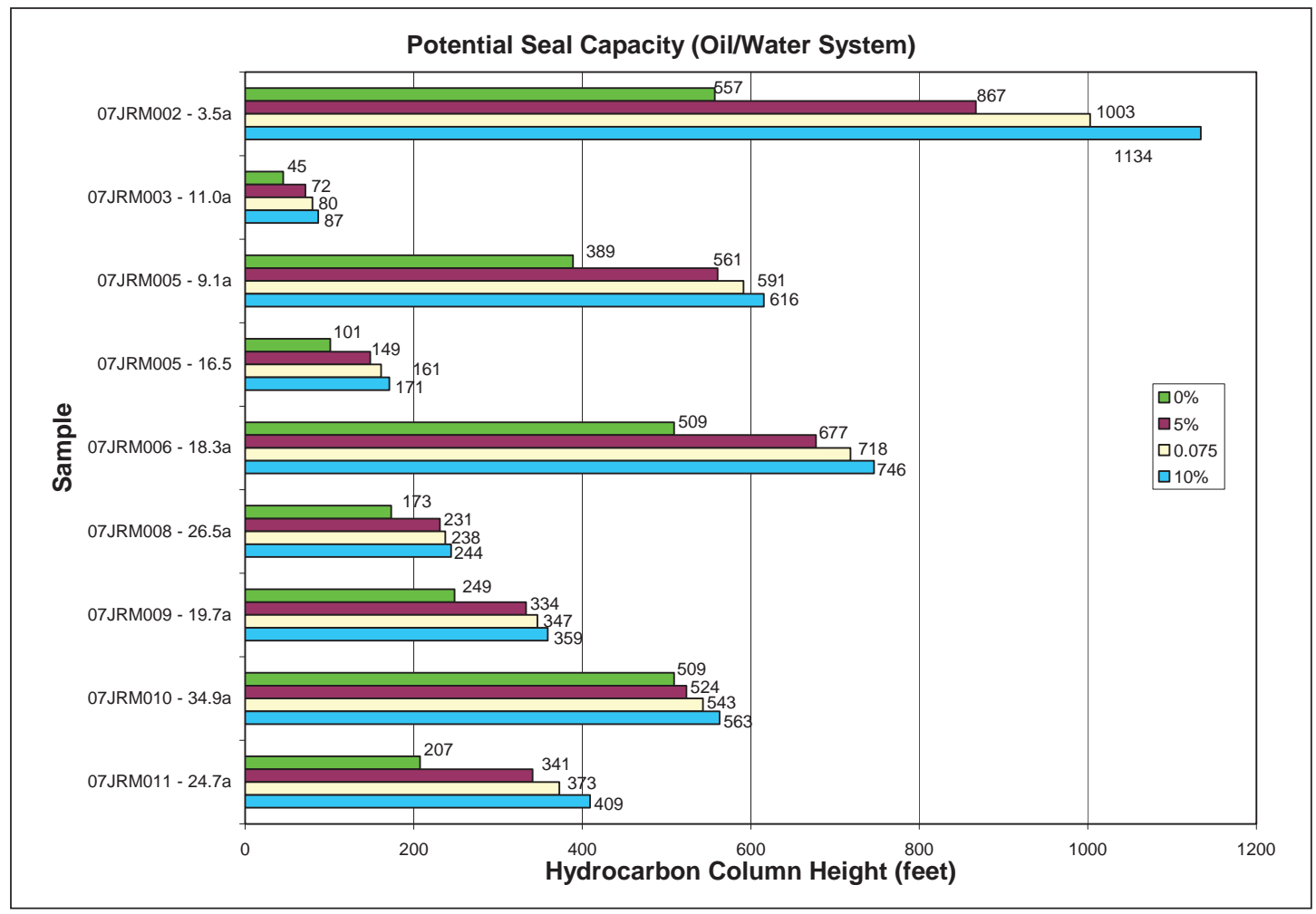

Figure 4a. Potential seal capacity in an oil/water system.

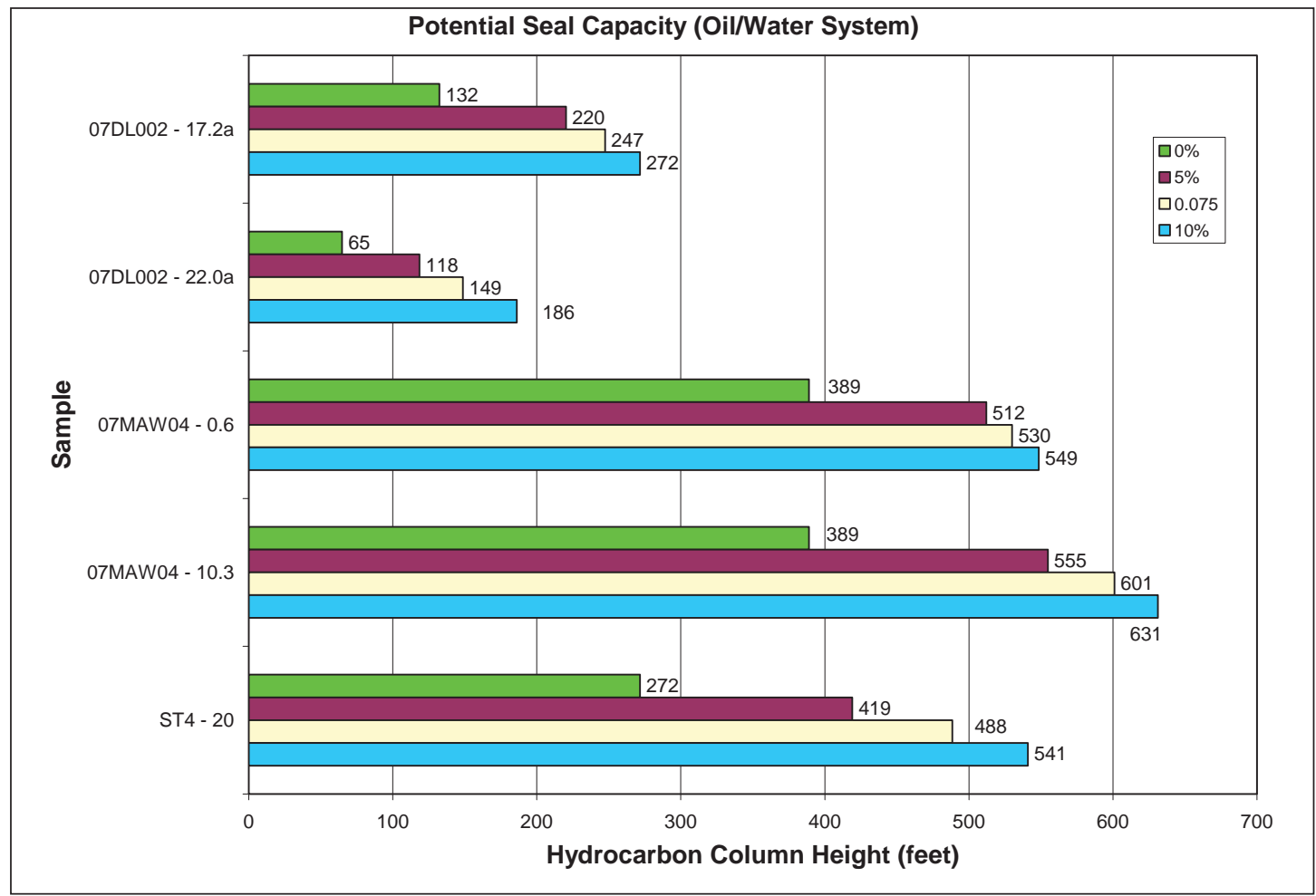

Figure 4b. Potential seal capacity in an oil/water system. 


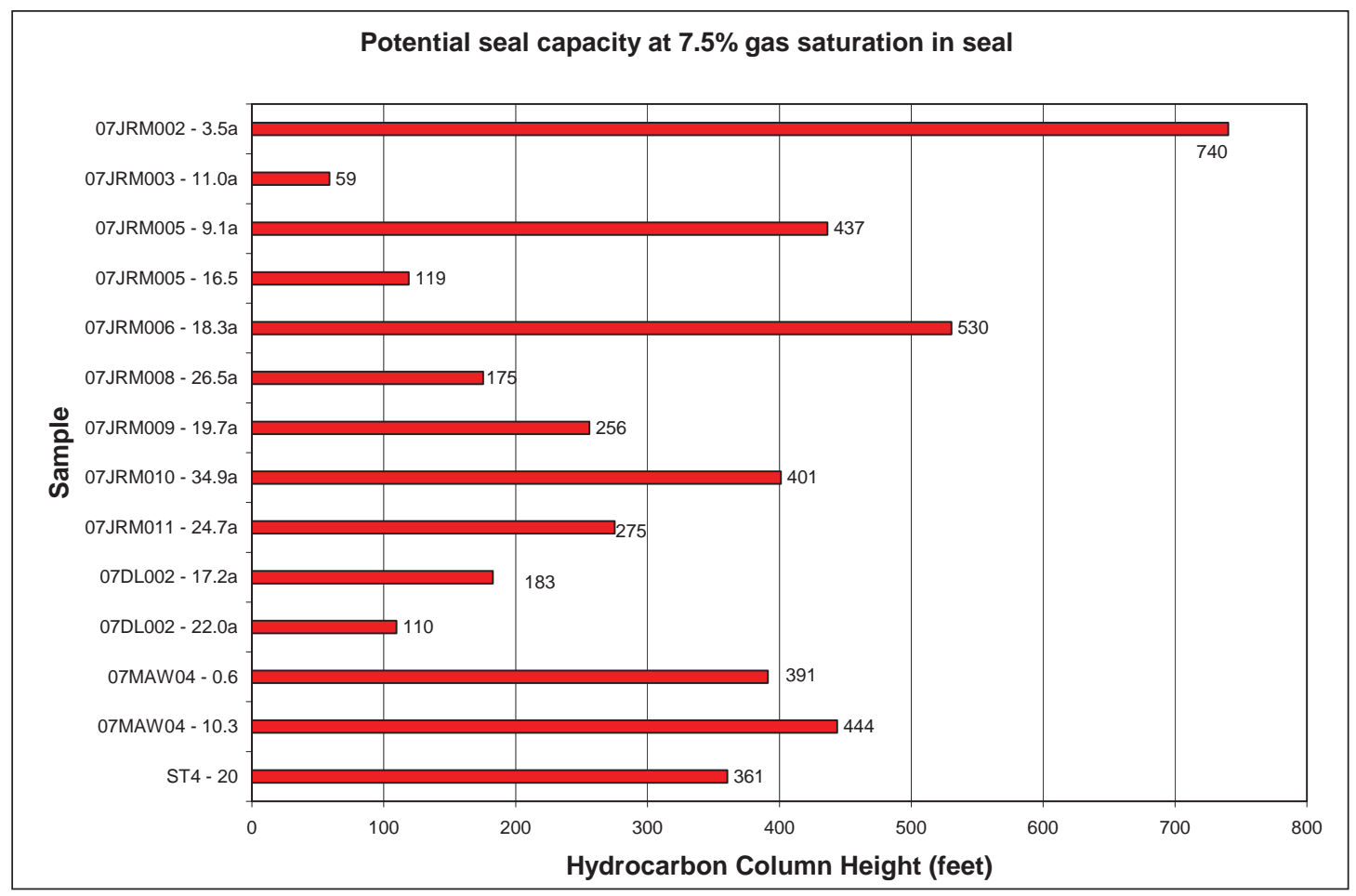

Figure 5. Chart of potential seal capacity at $7.5 \%$ gas saturation in seal for each sample.

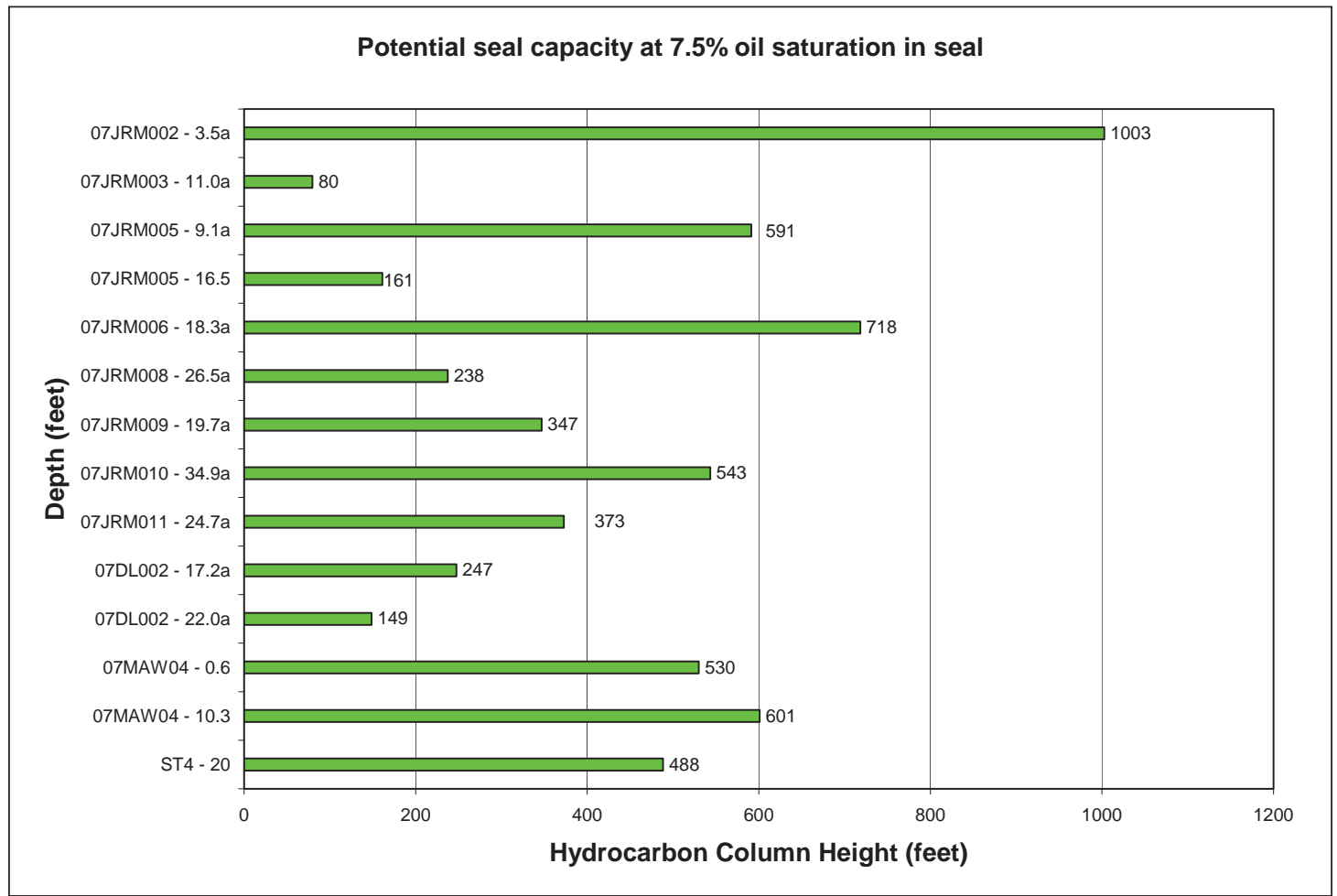

Figure 6. Chart of potential seal capacity at 7.5\% oil saturation in seal for each sample. 

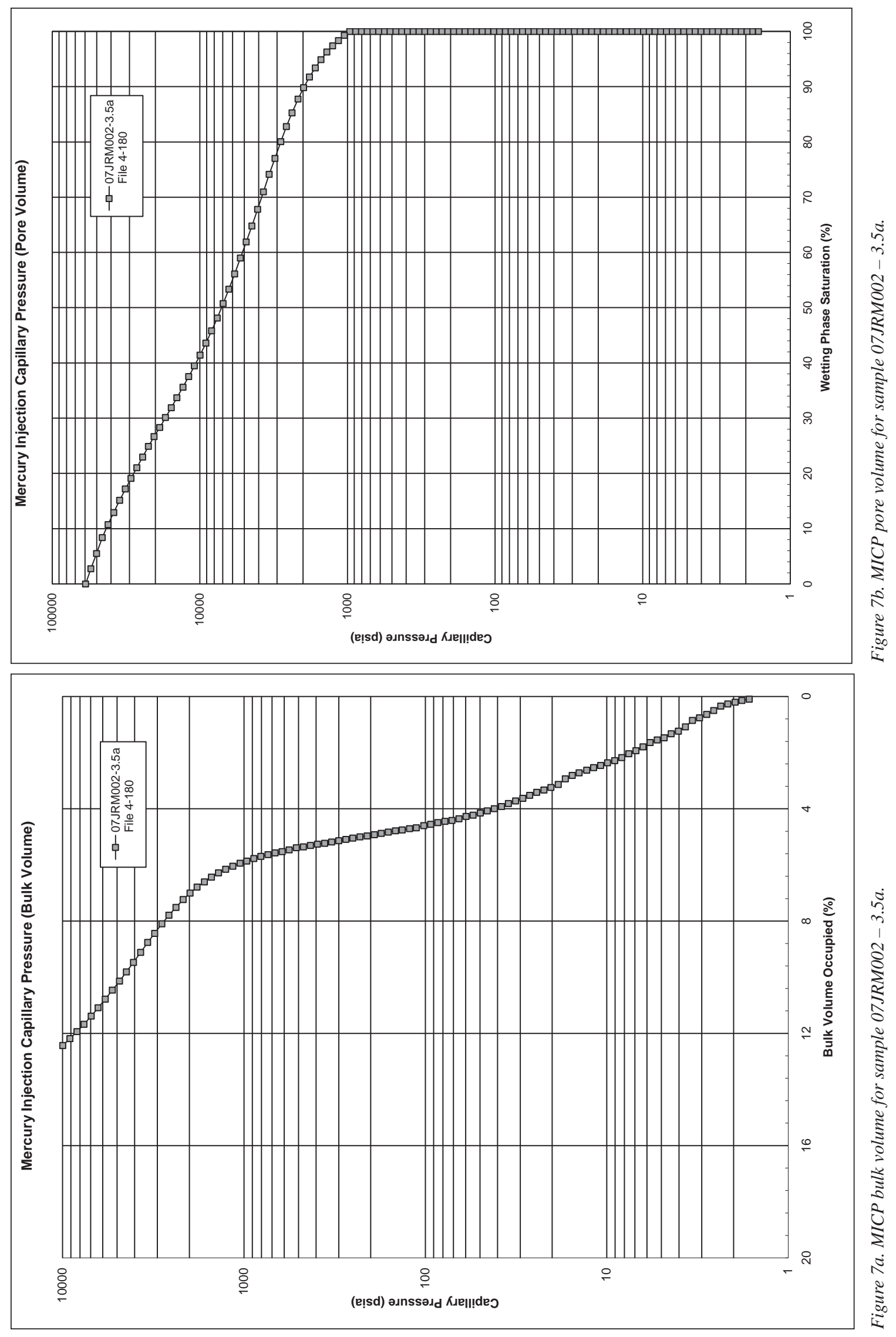


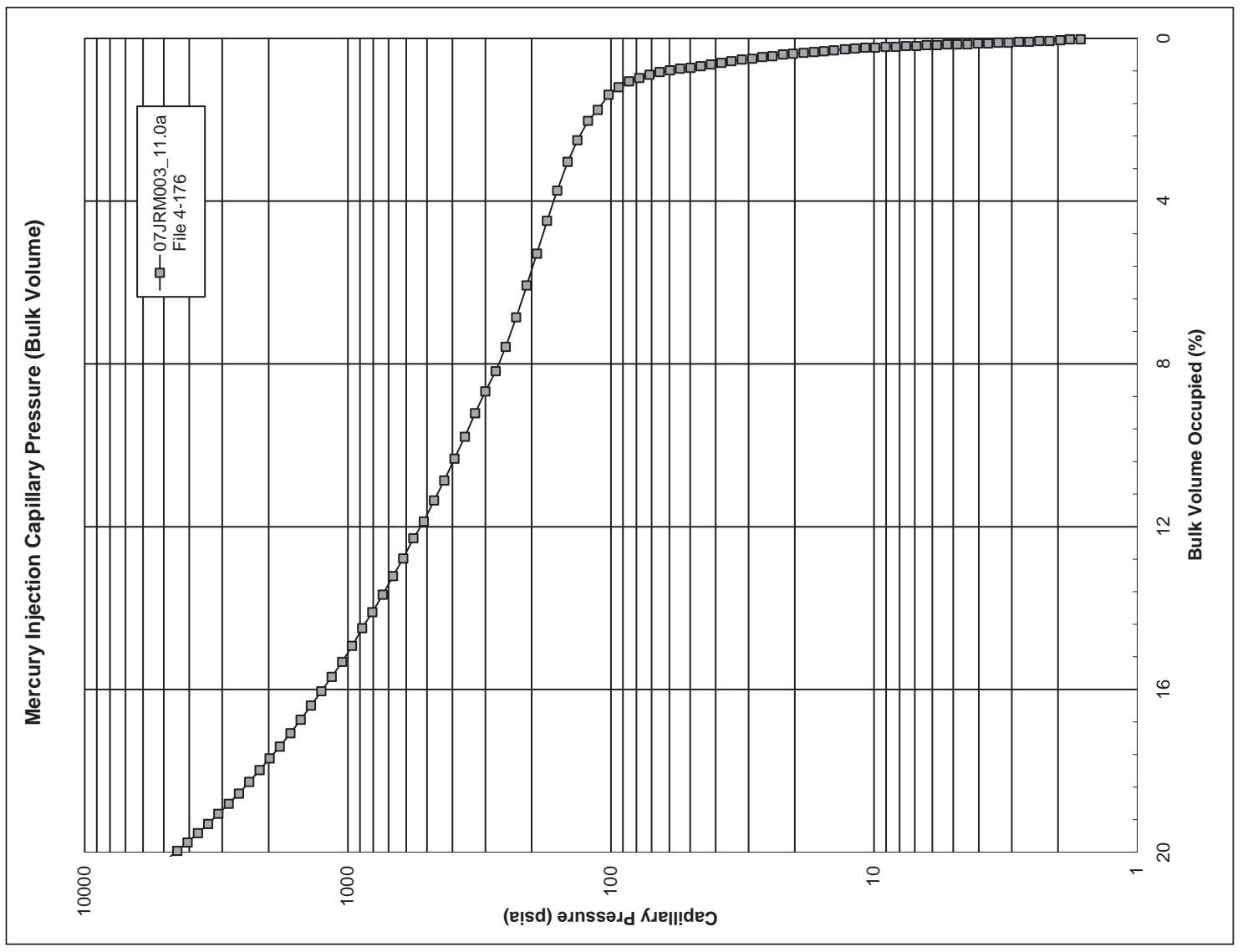

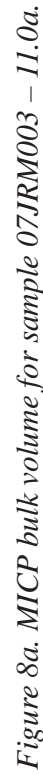

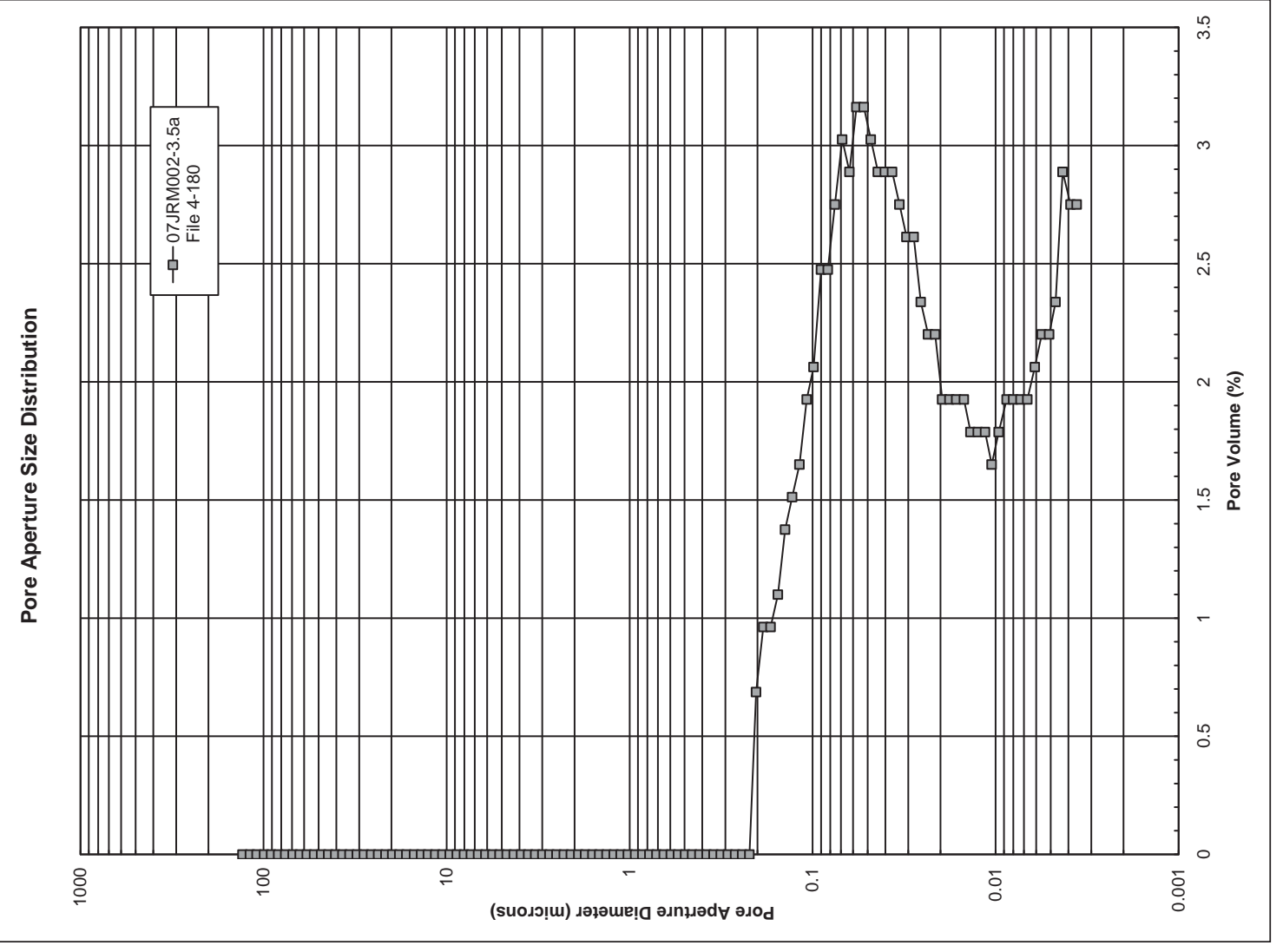

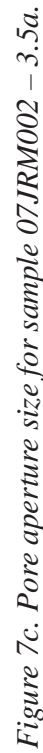



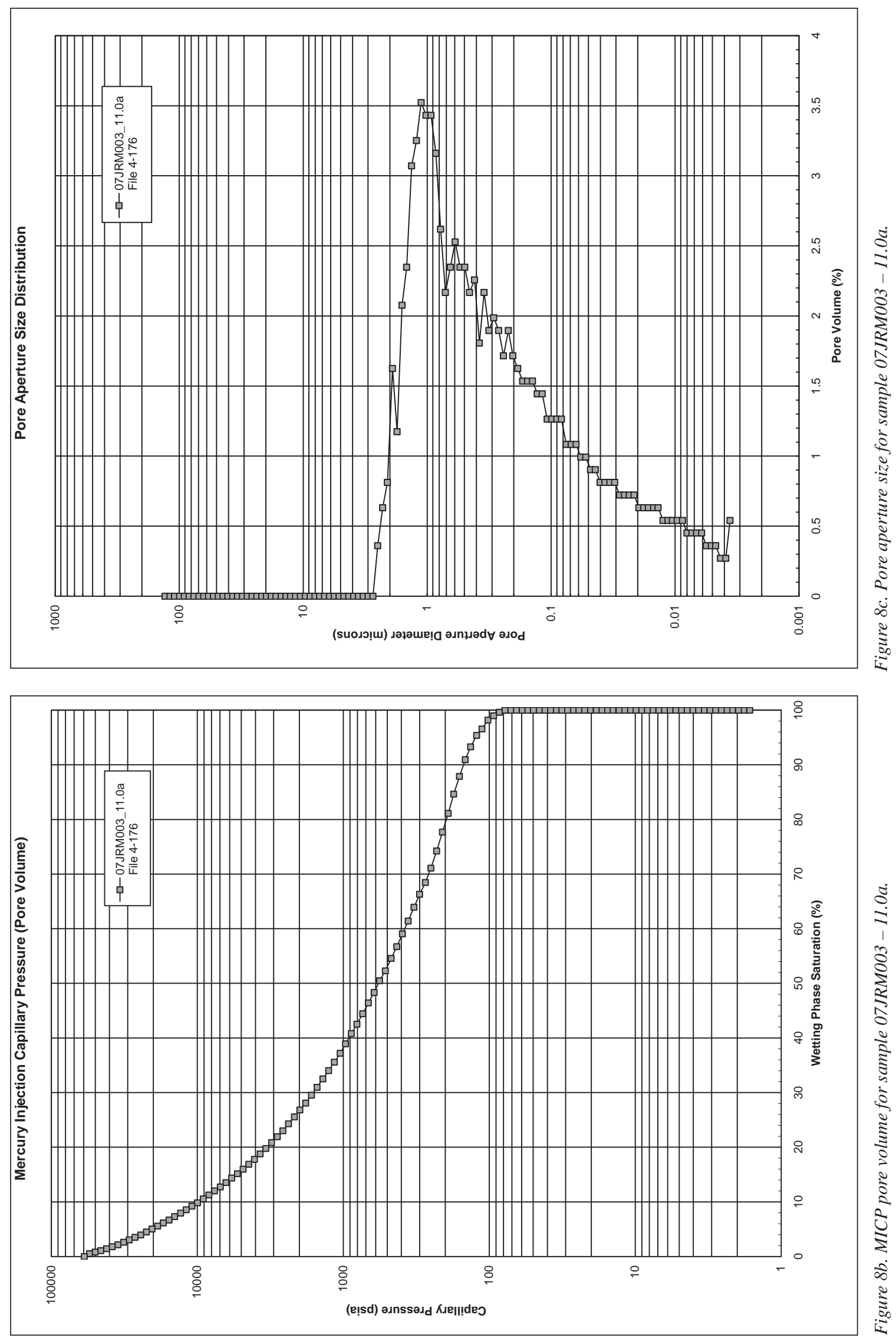


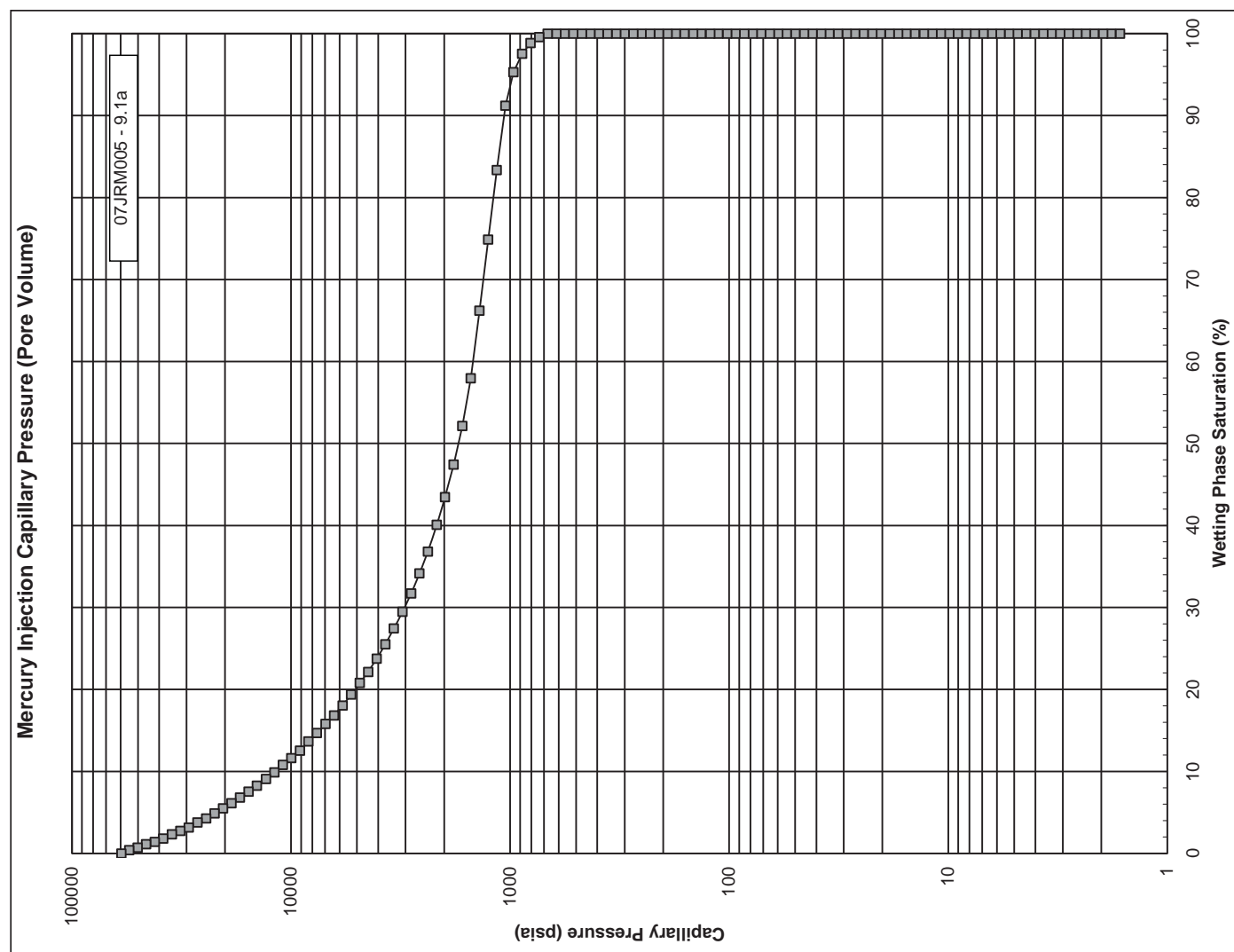

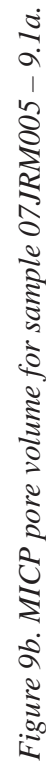

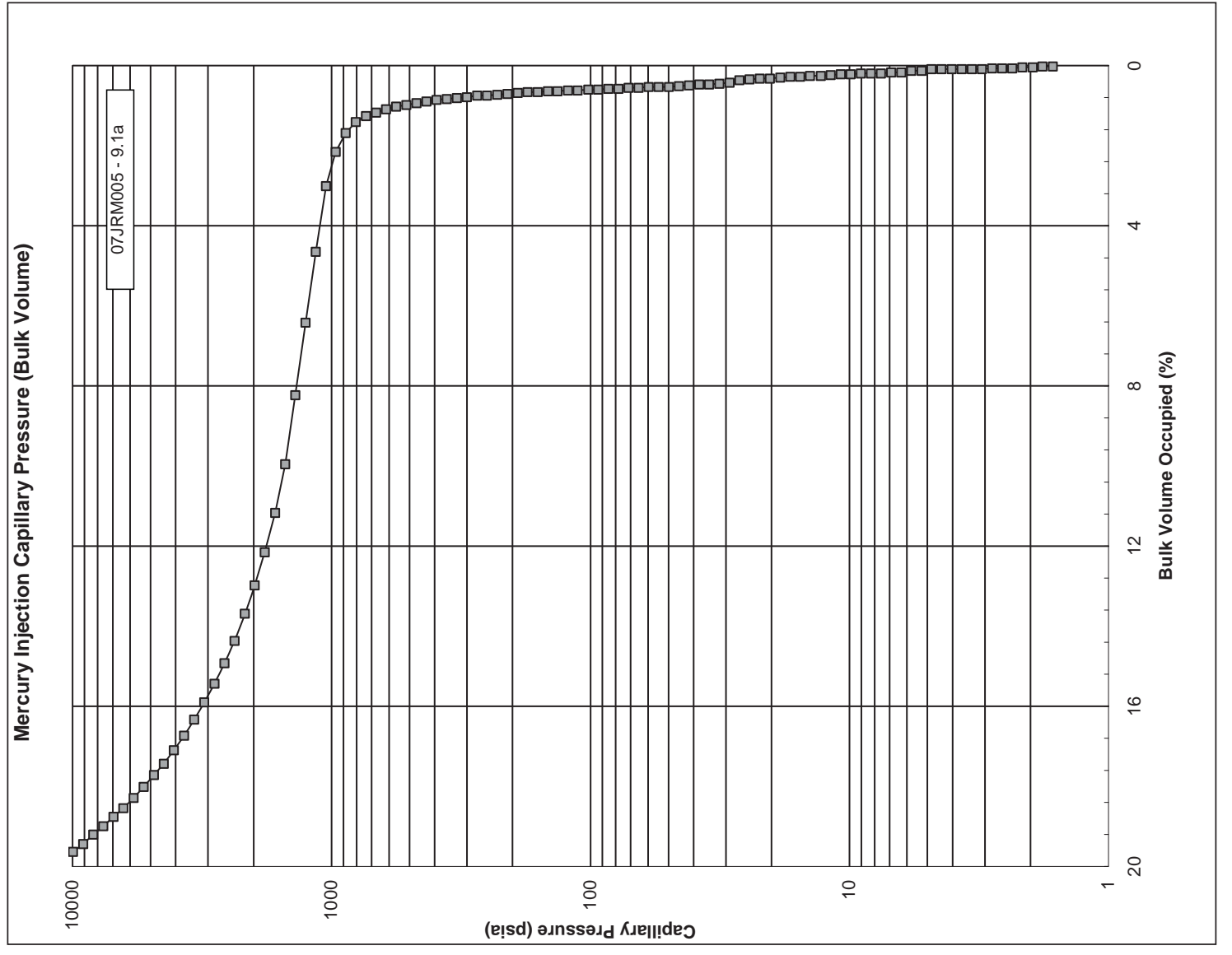

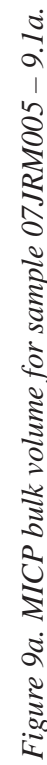



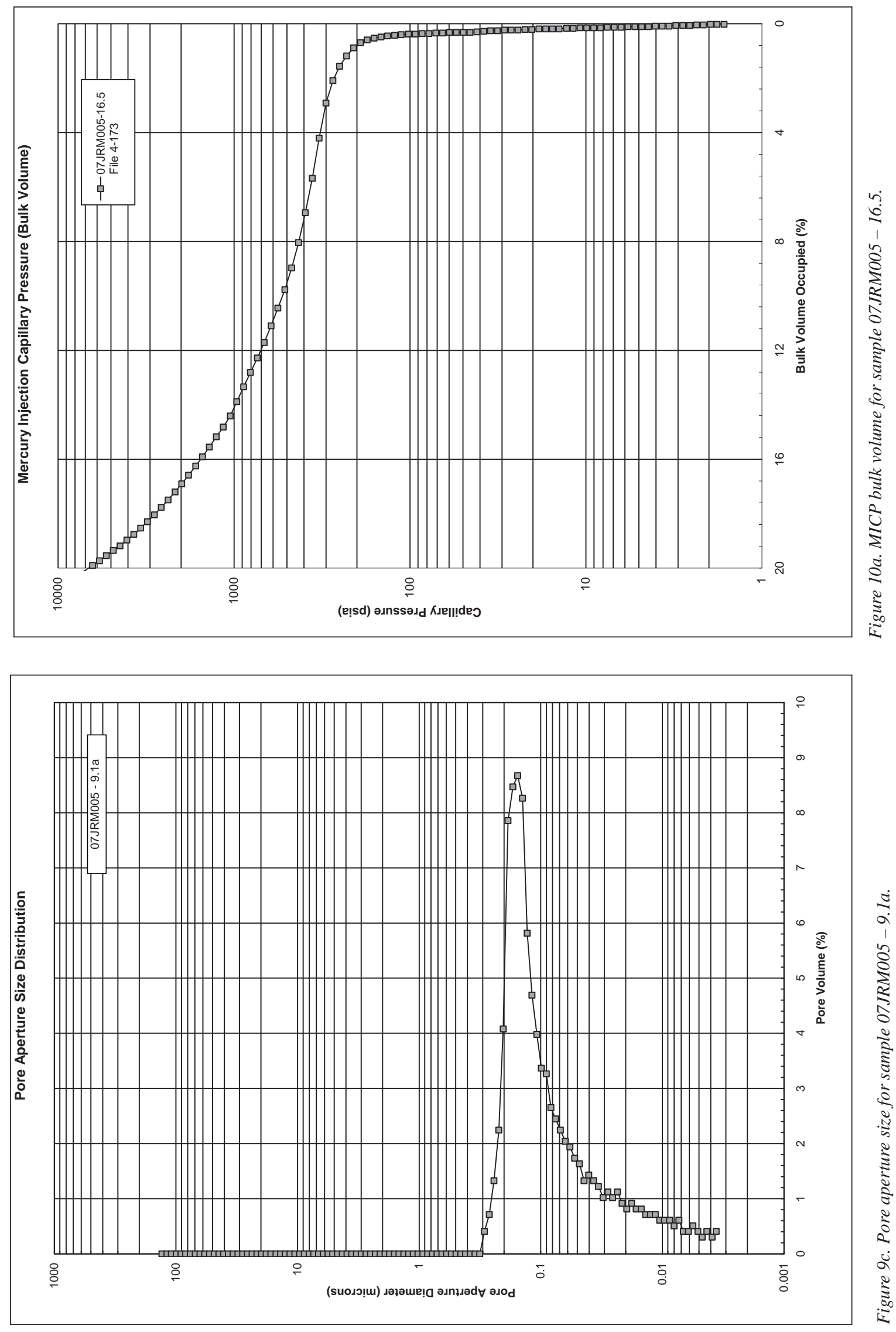


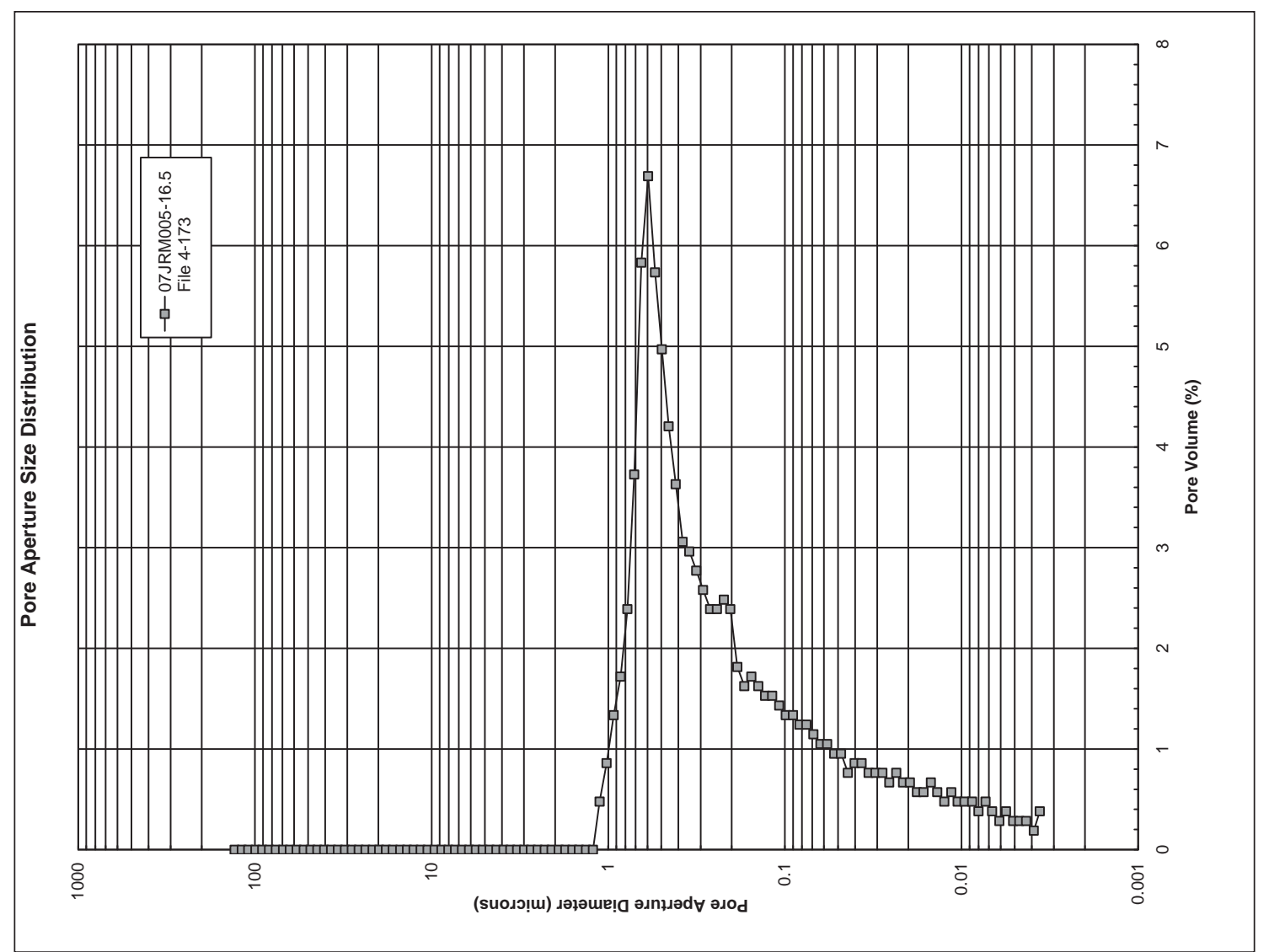

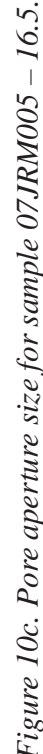

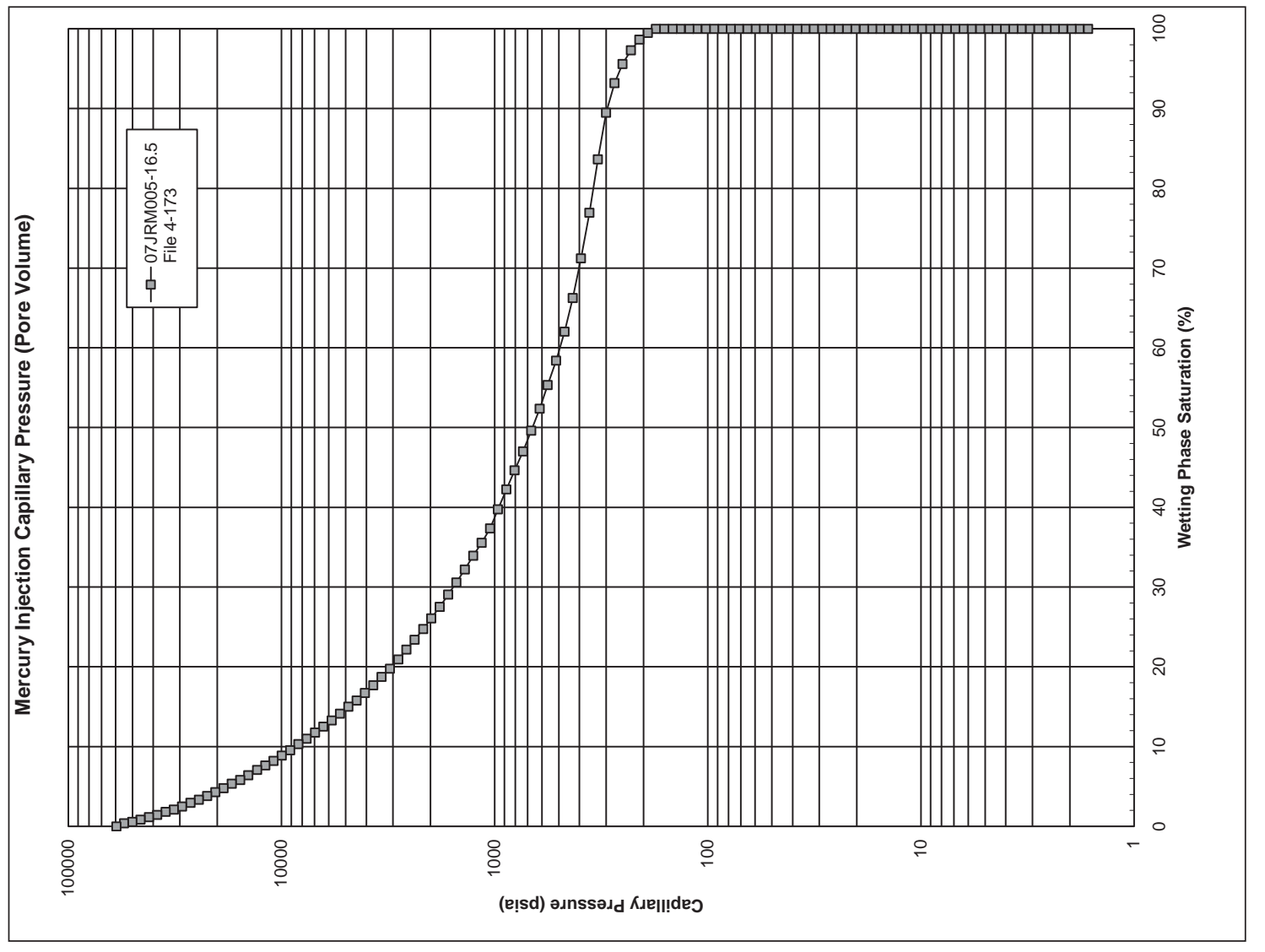

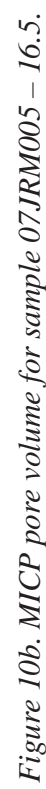



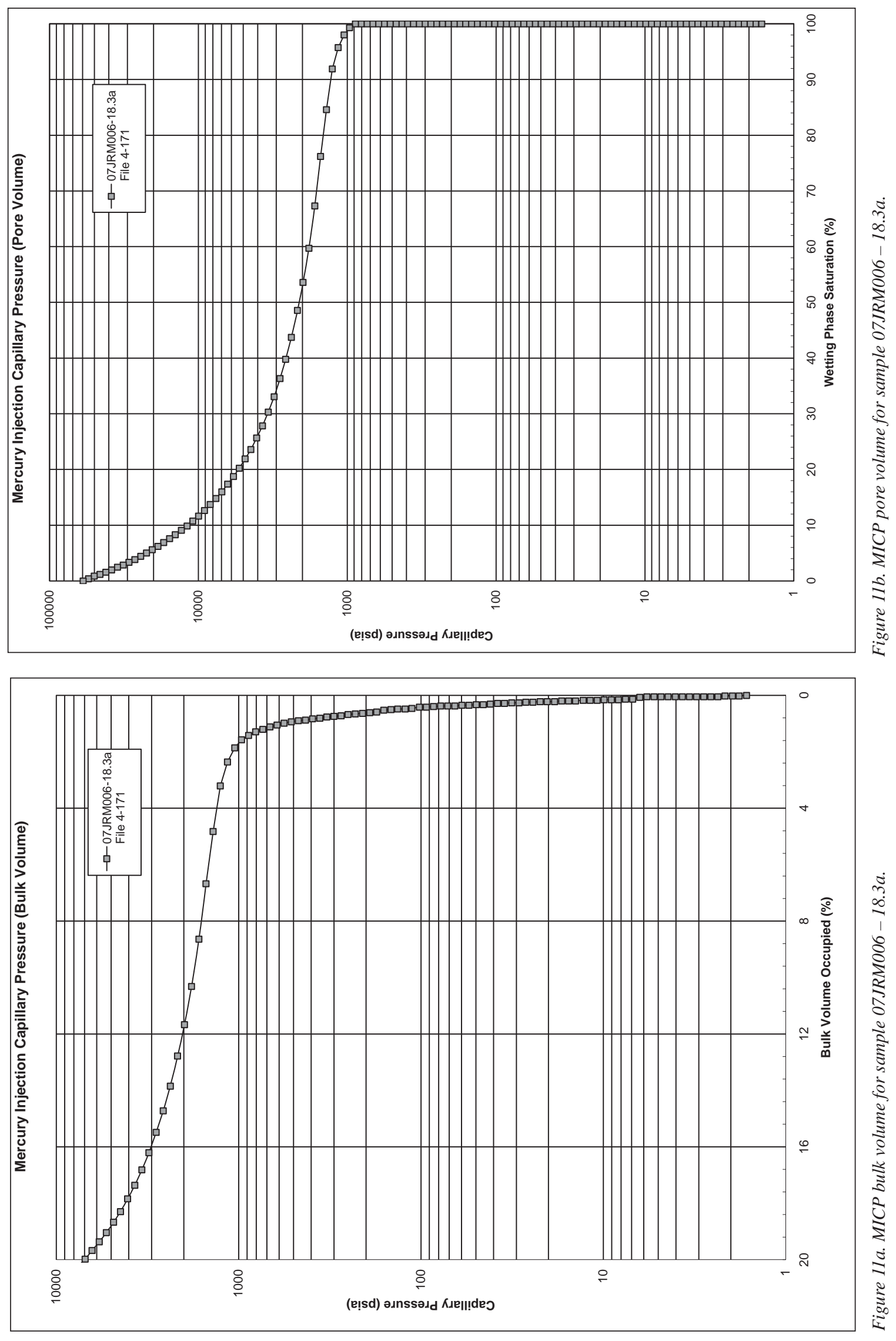


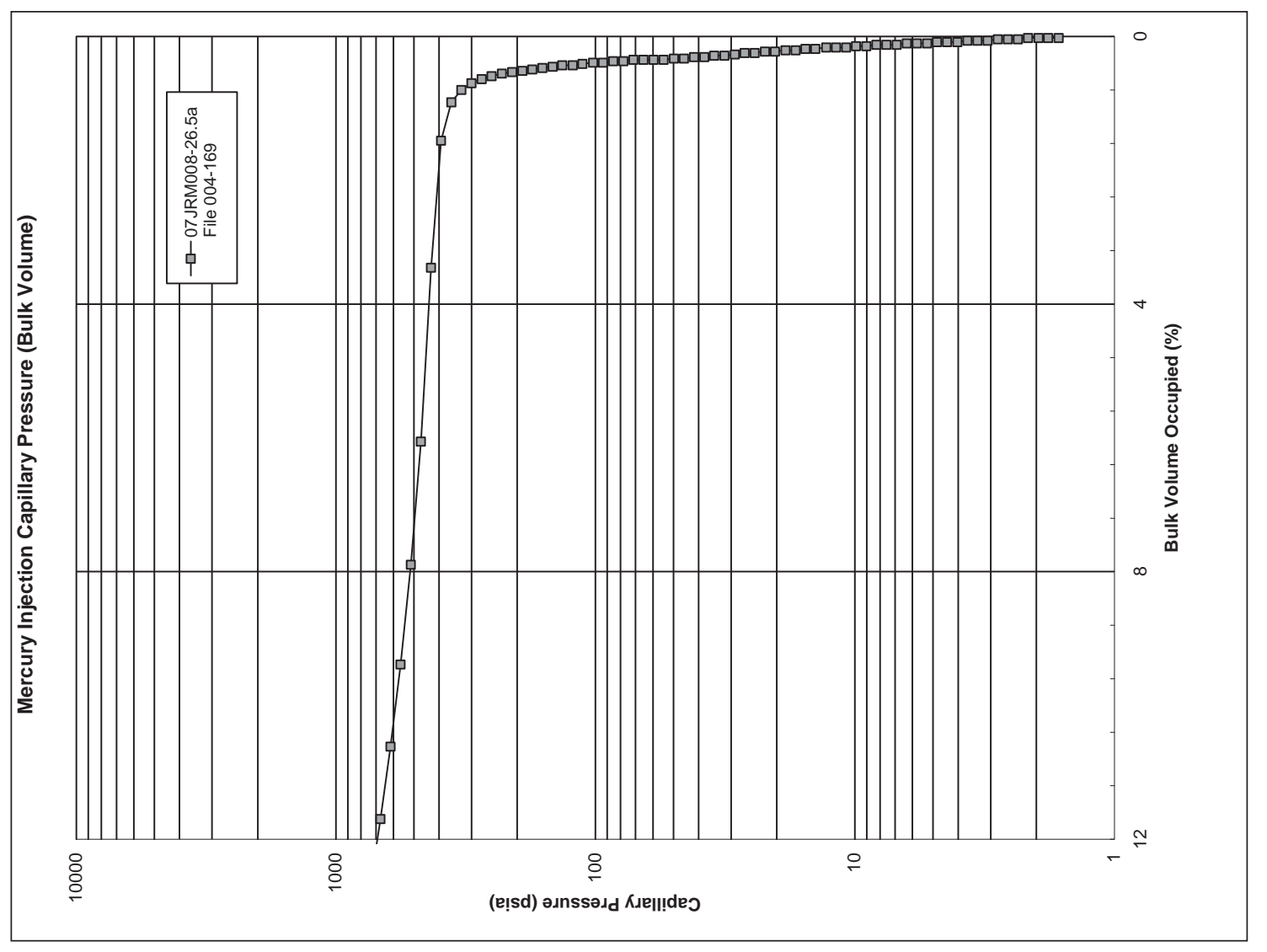

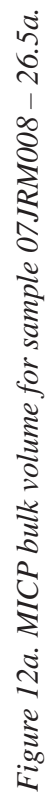

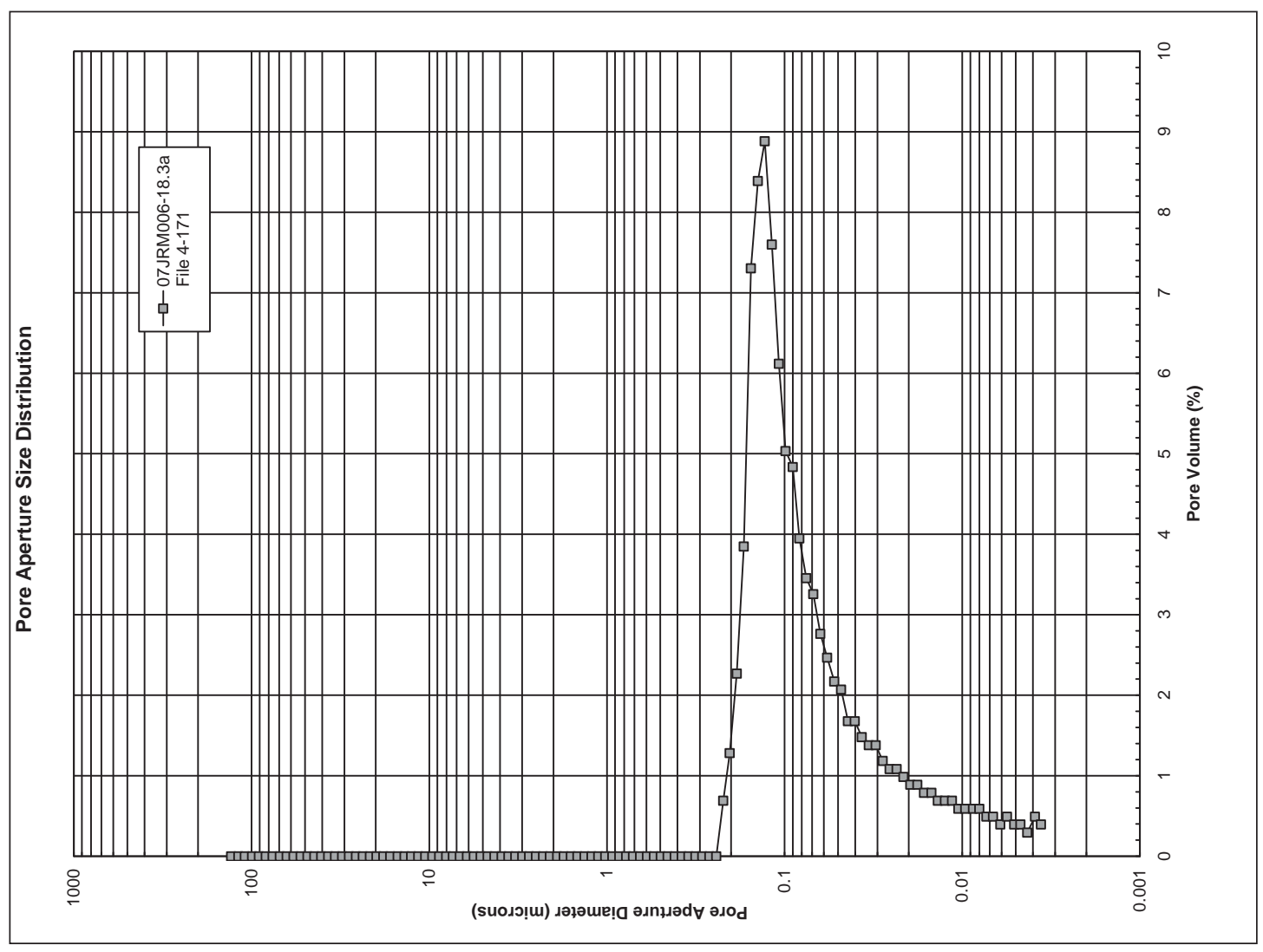

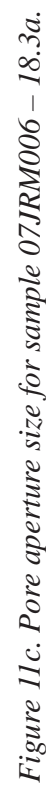



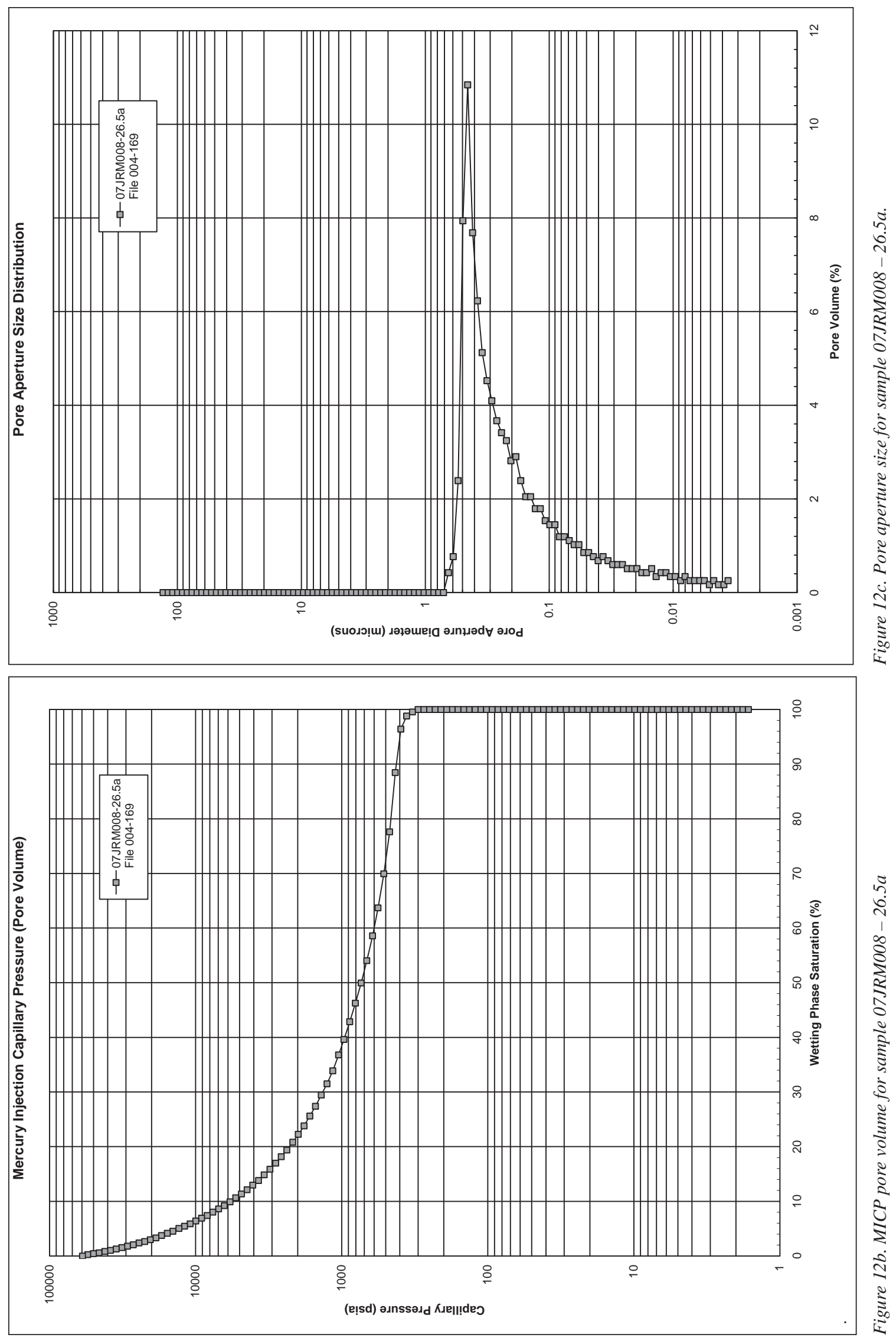


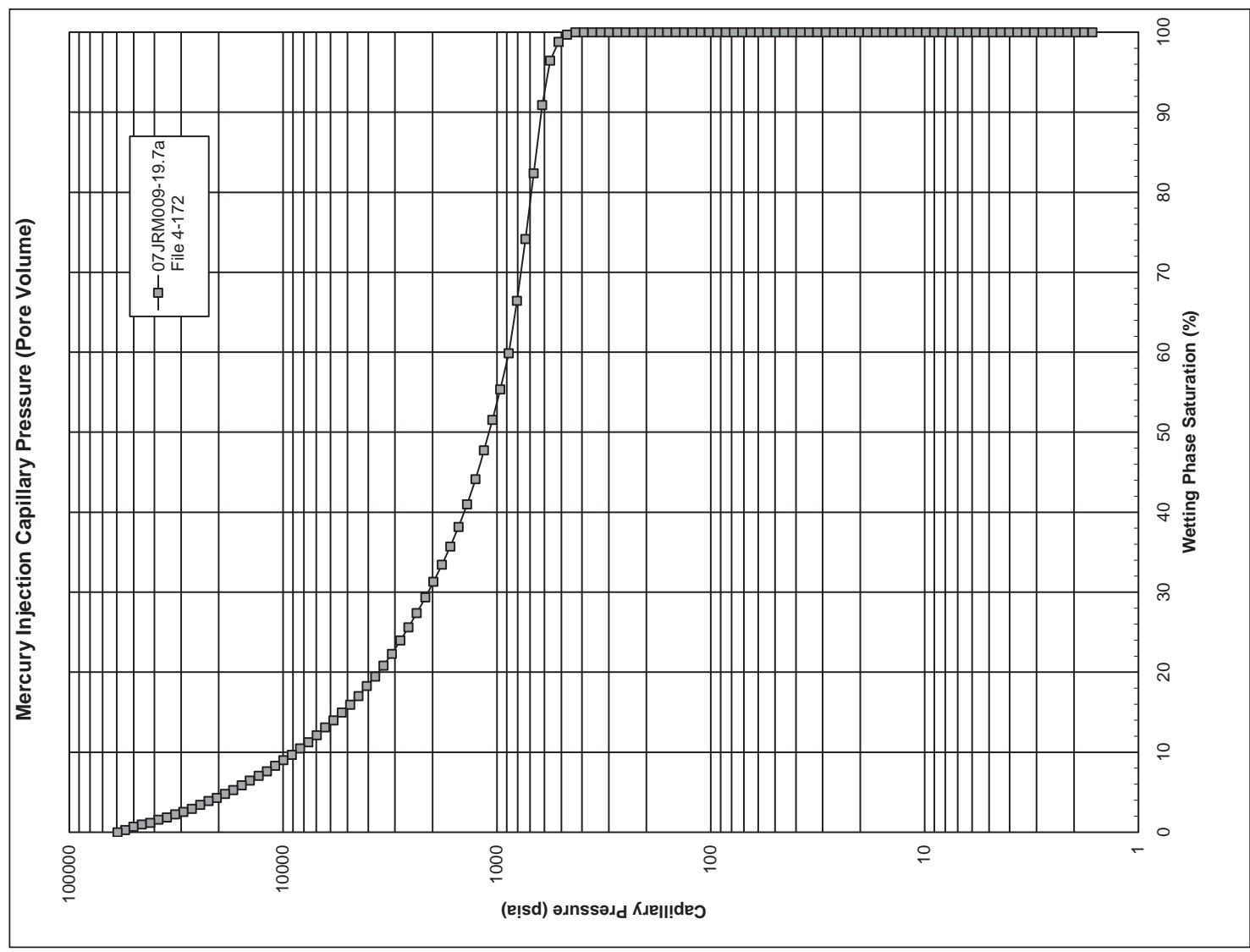

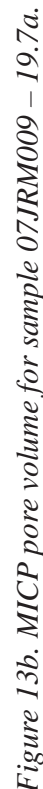

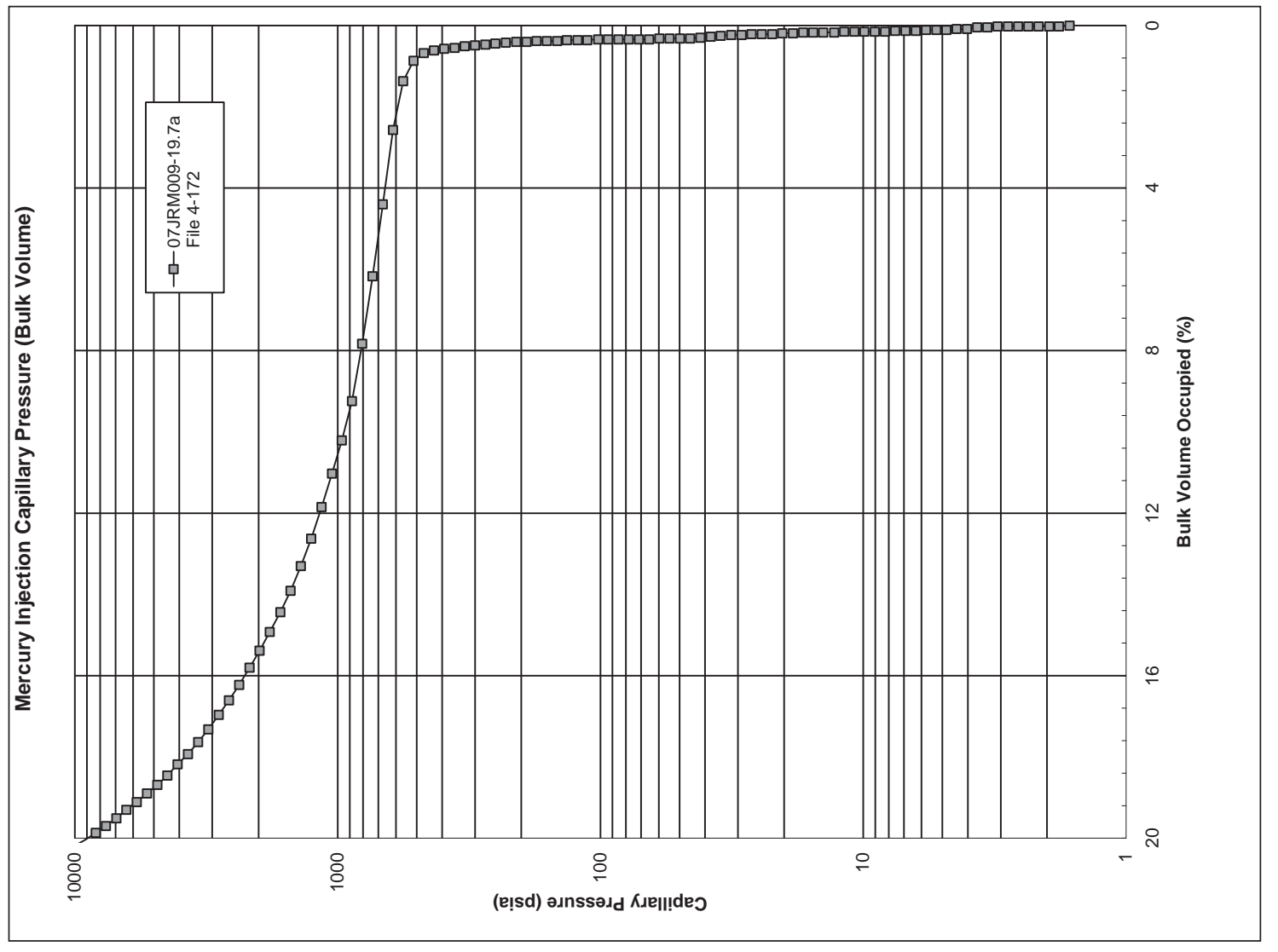

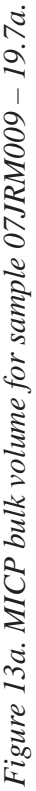



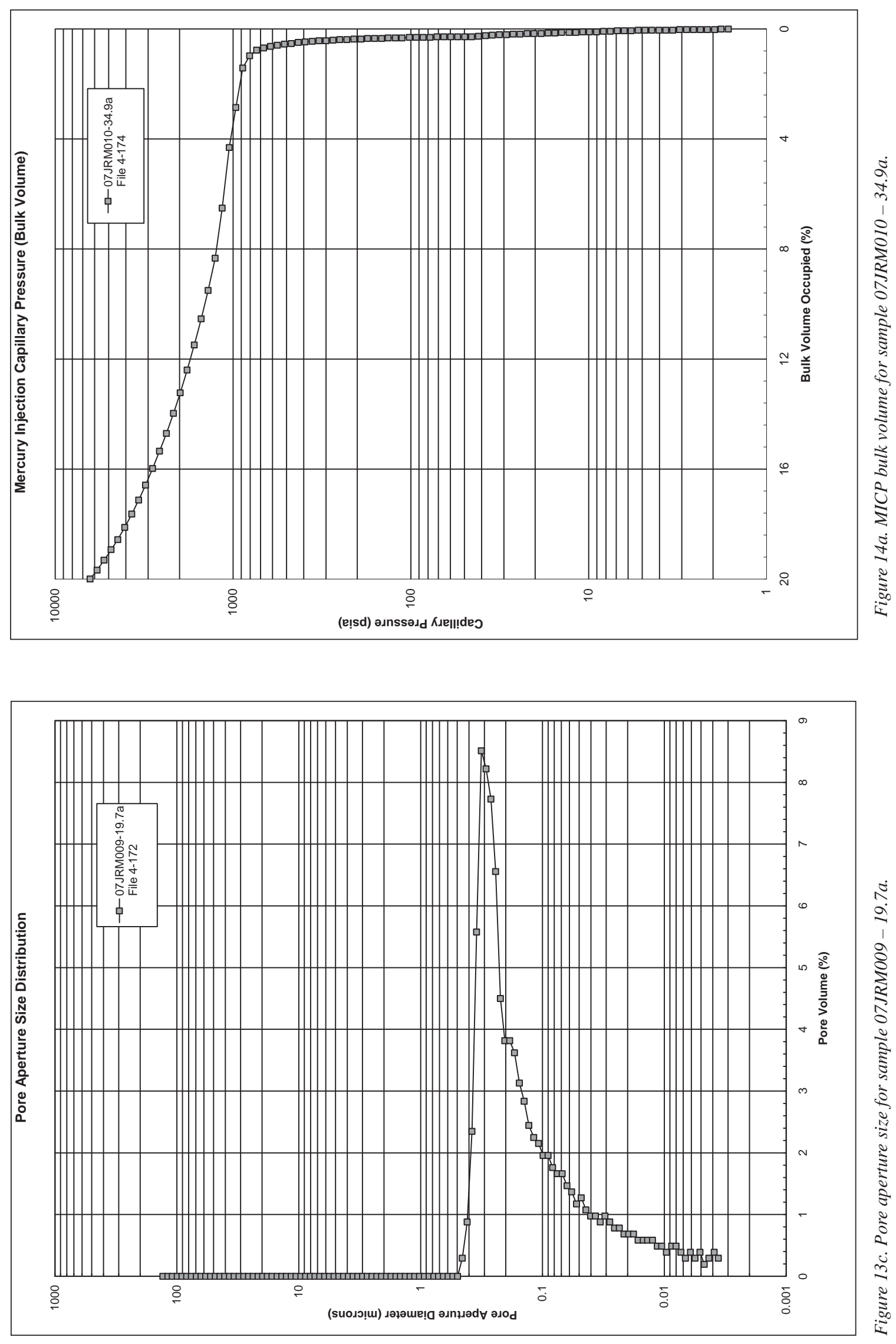


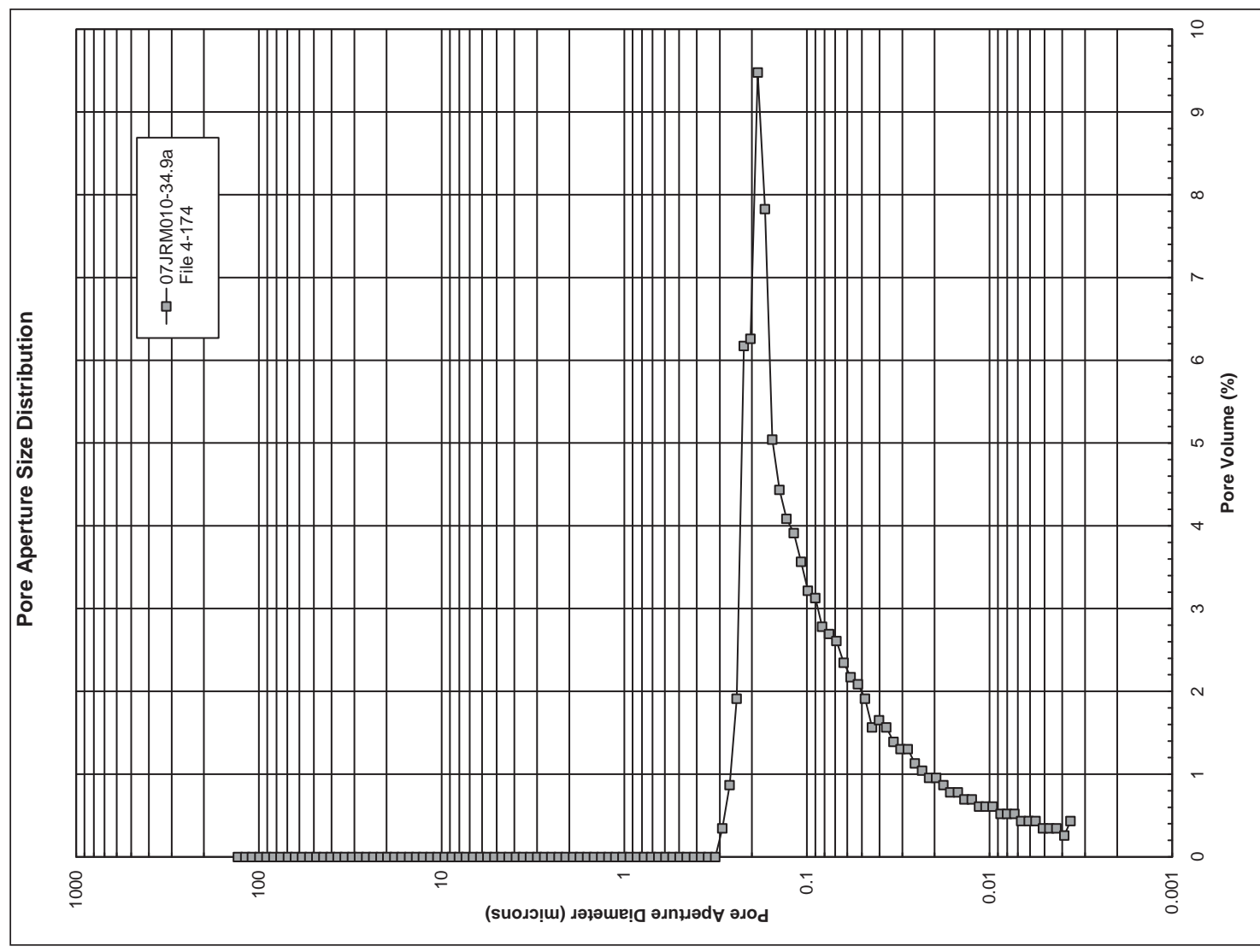

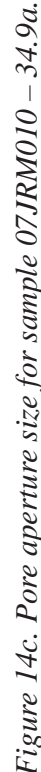

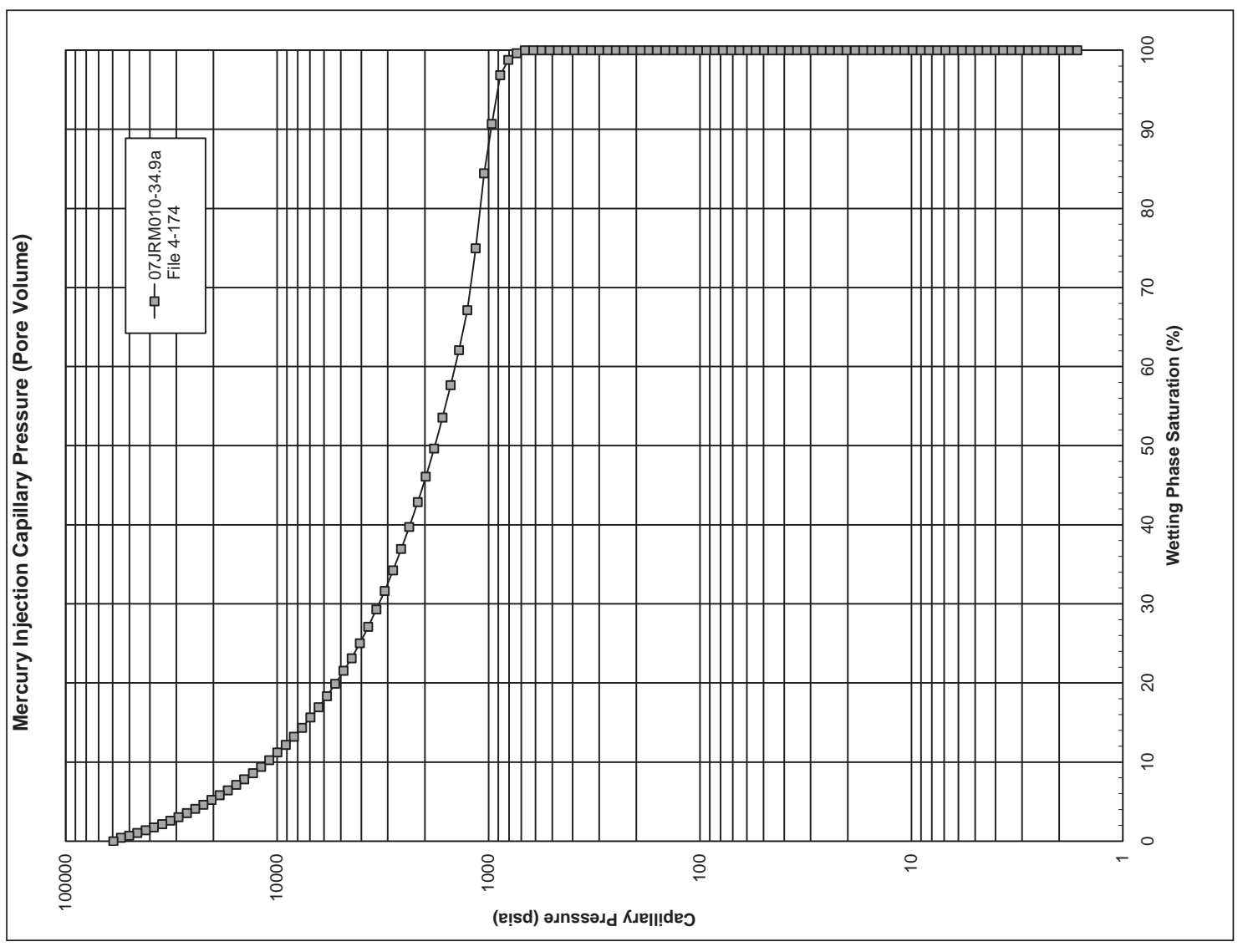

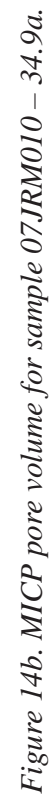




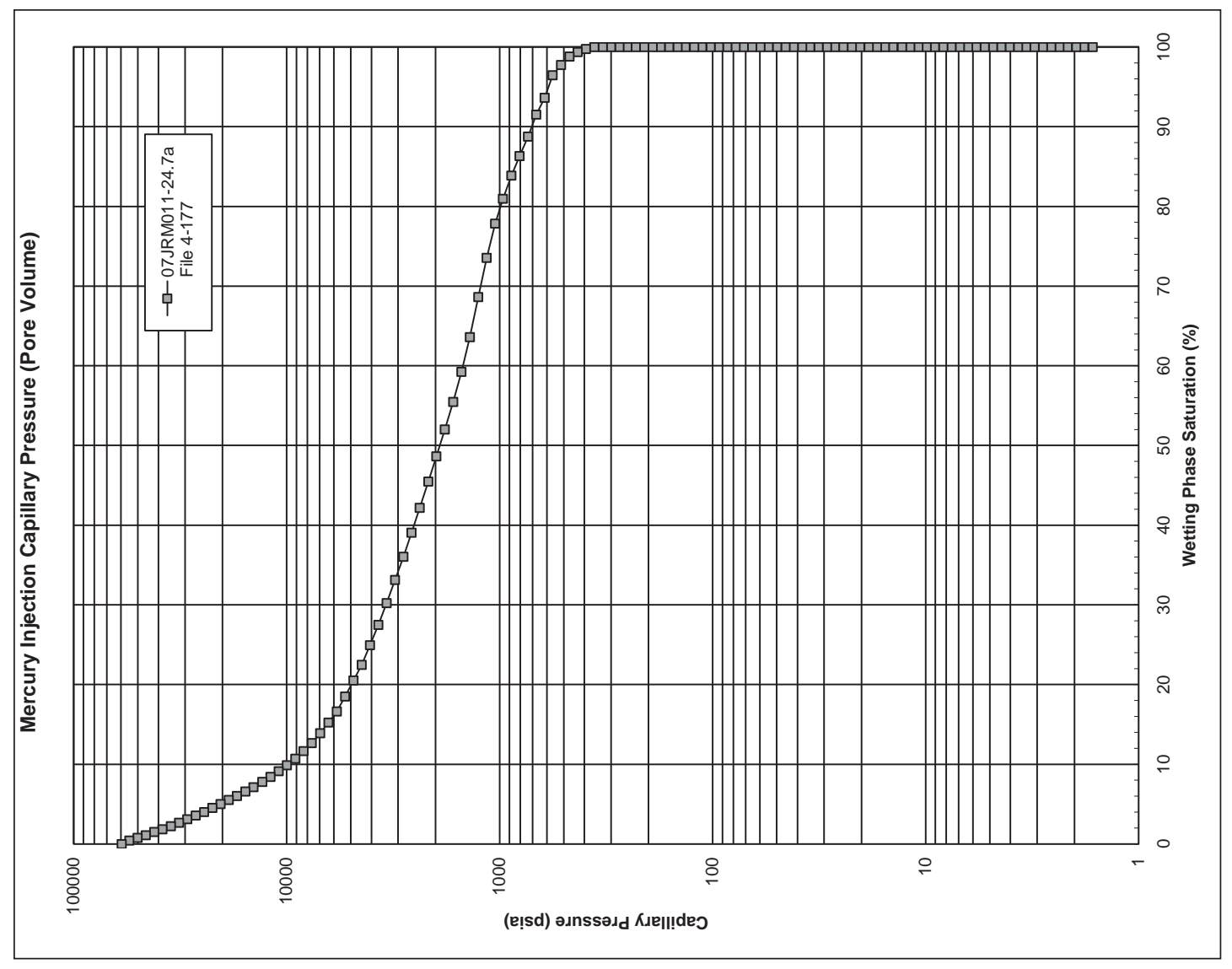

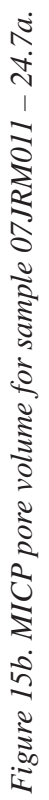

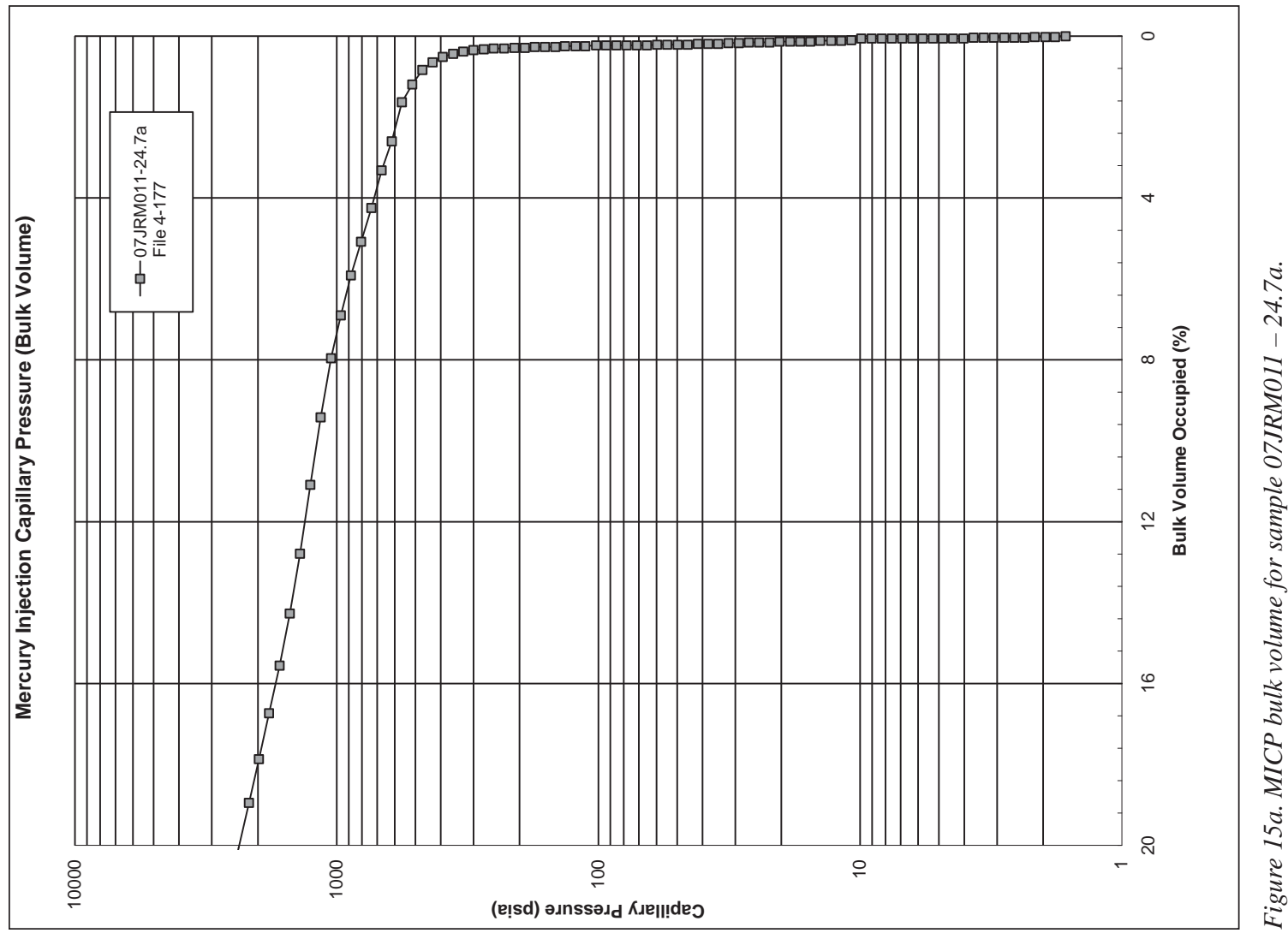



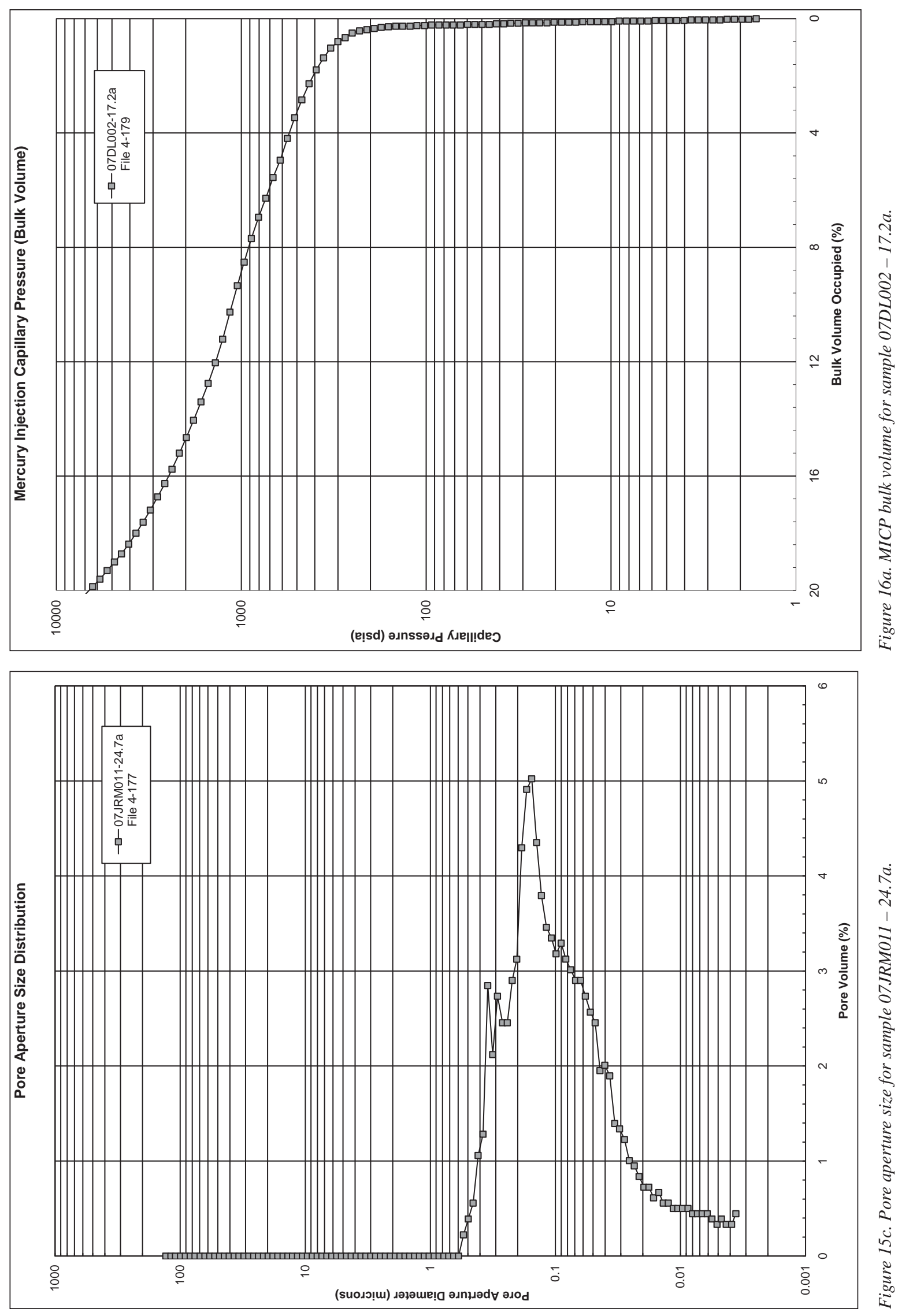

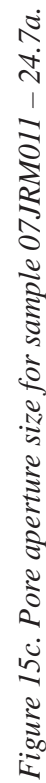



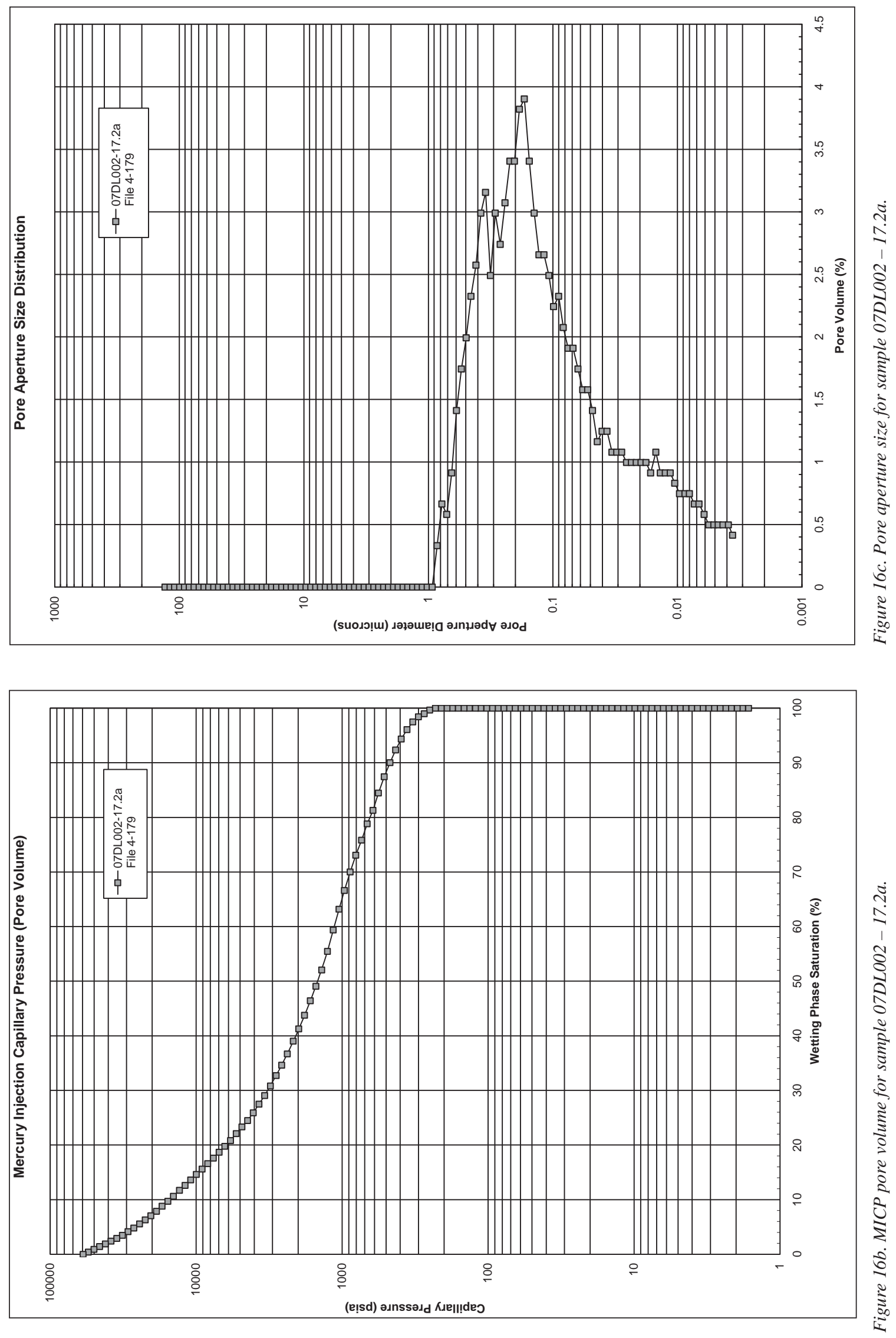


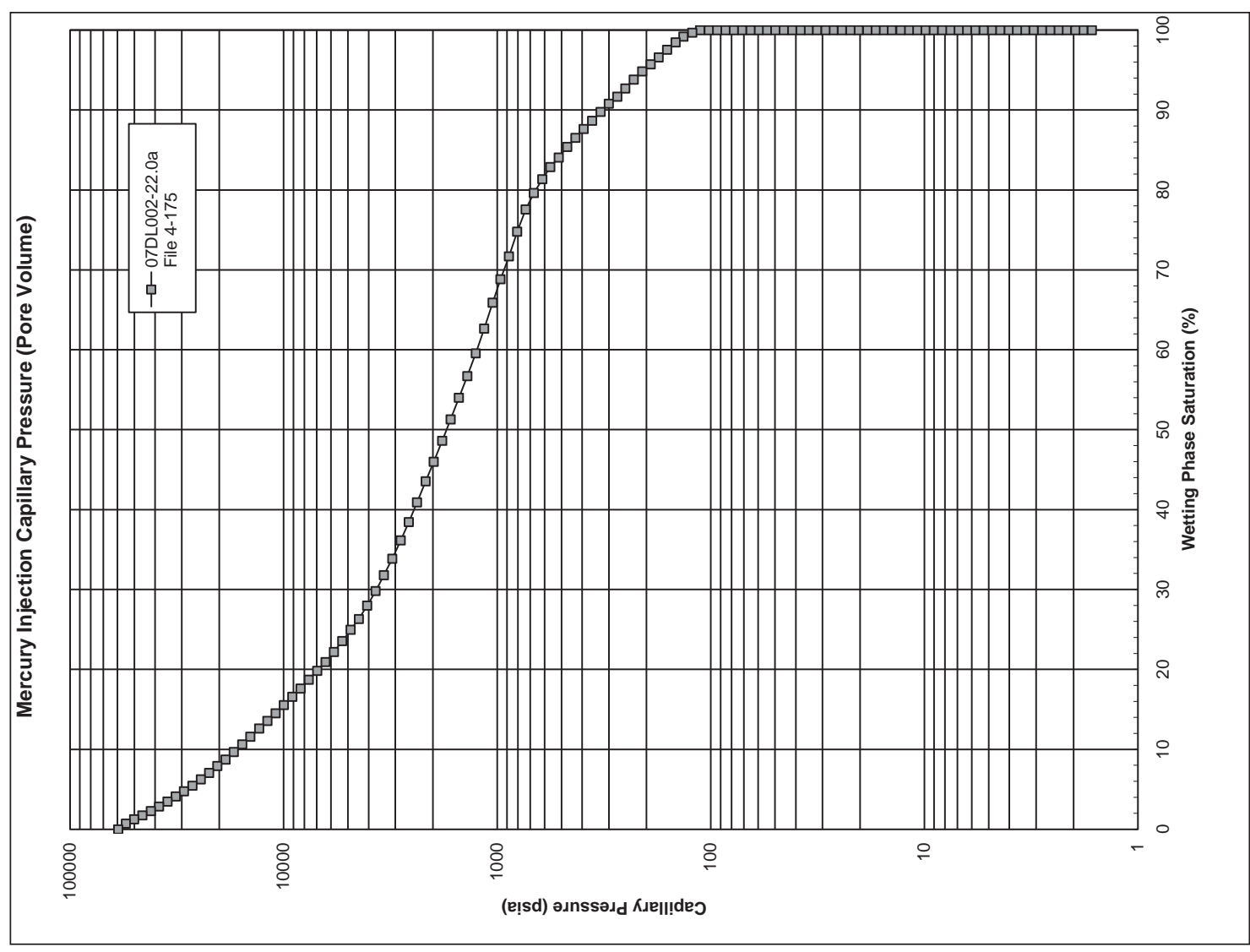

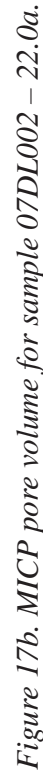

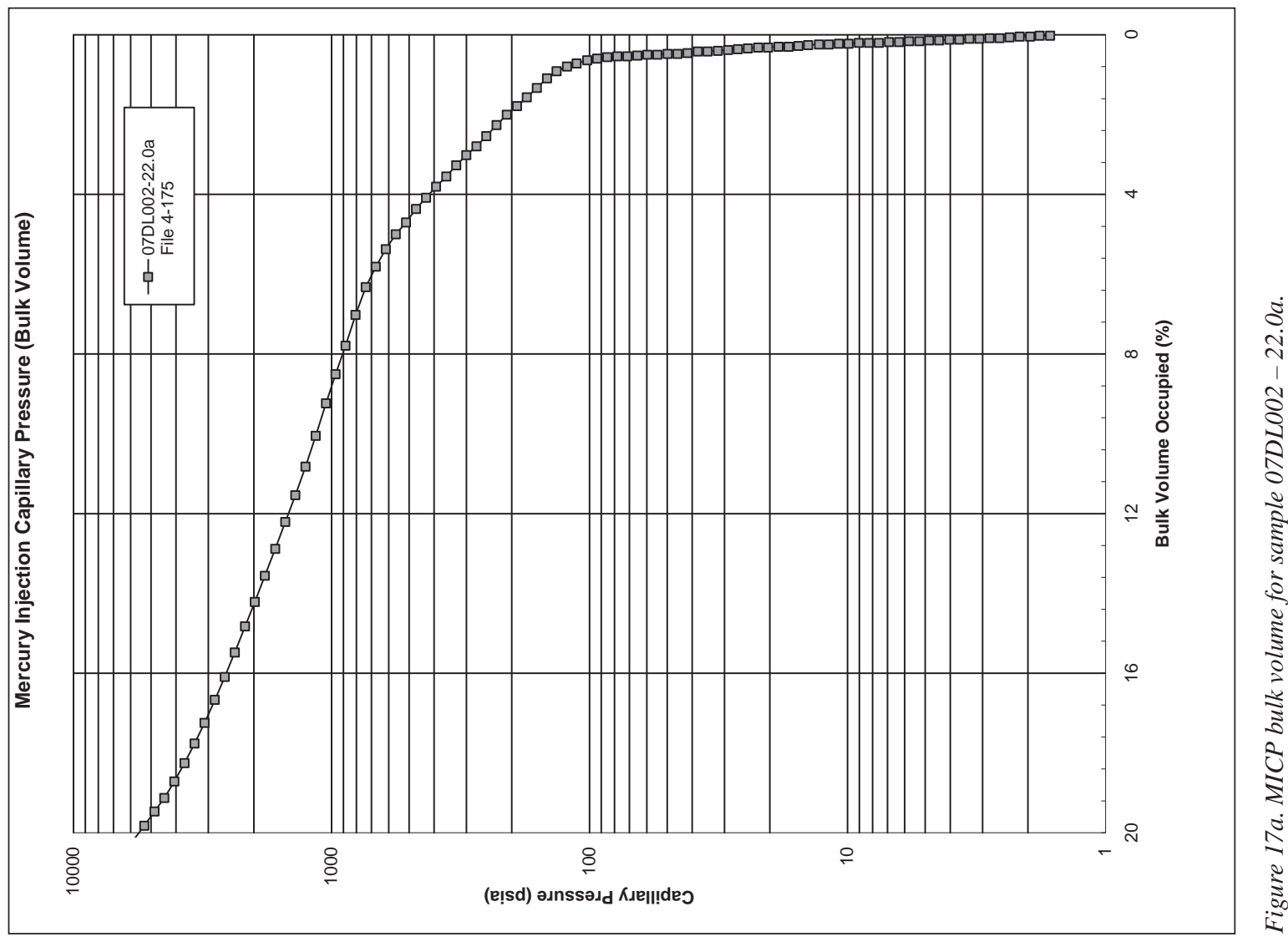




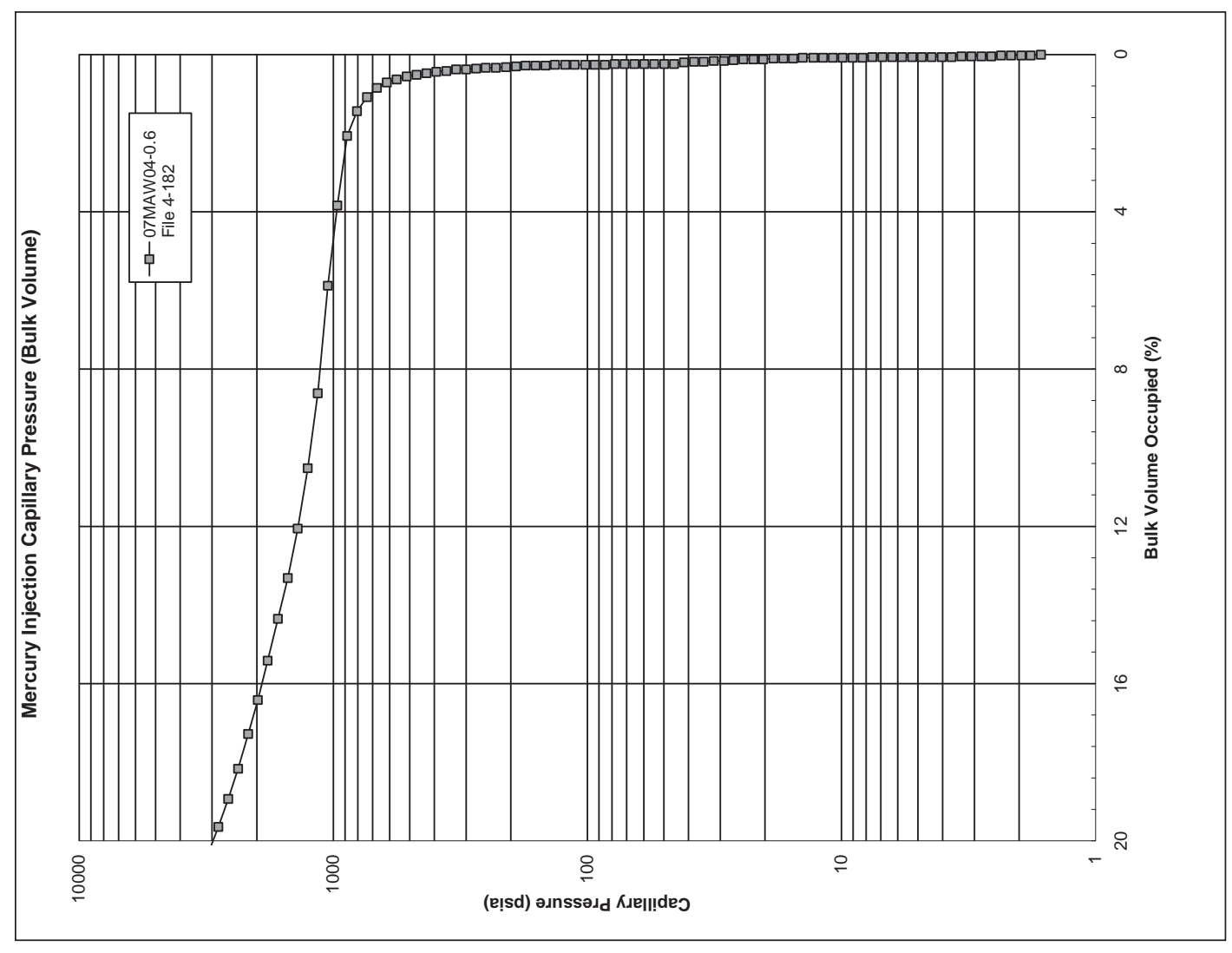

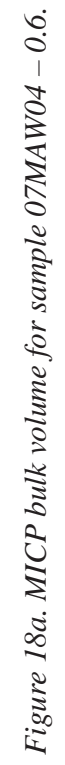

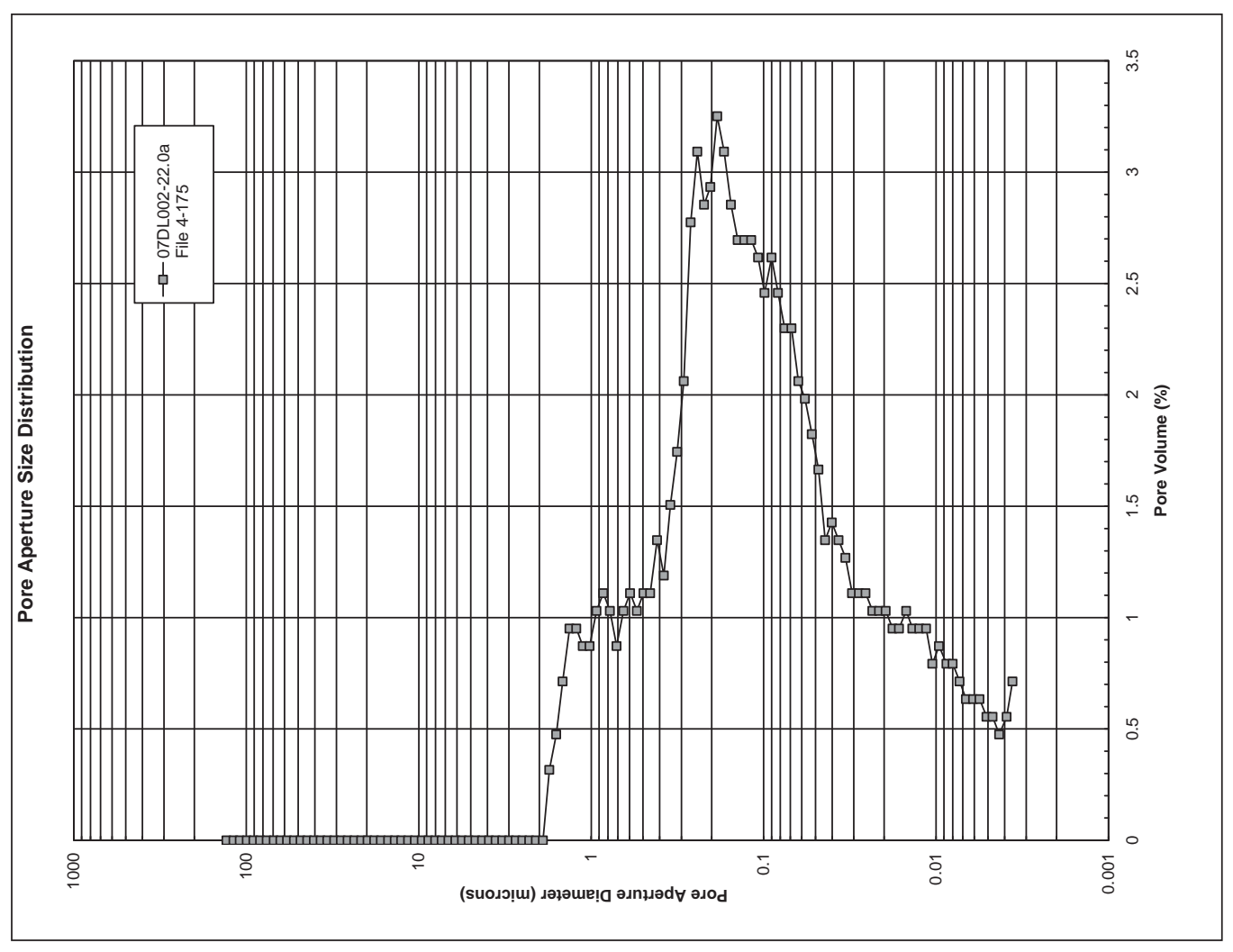

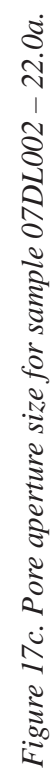




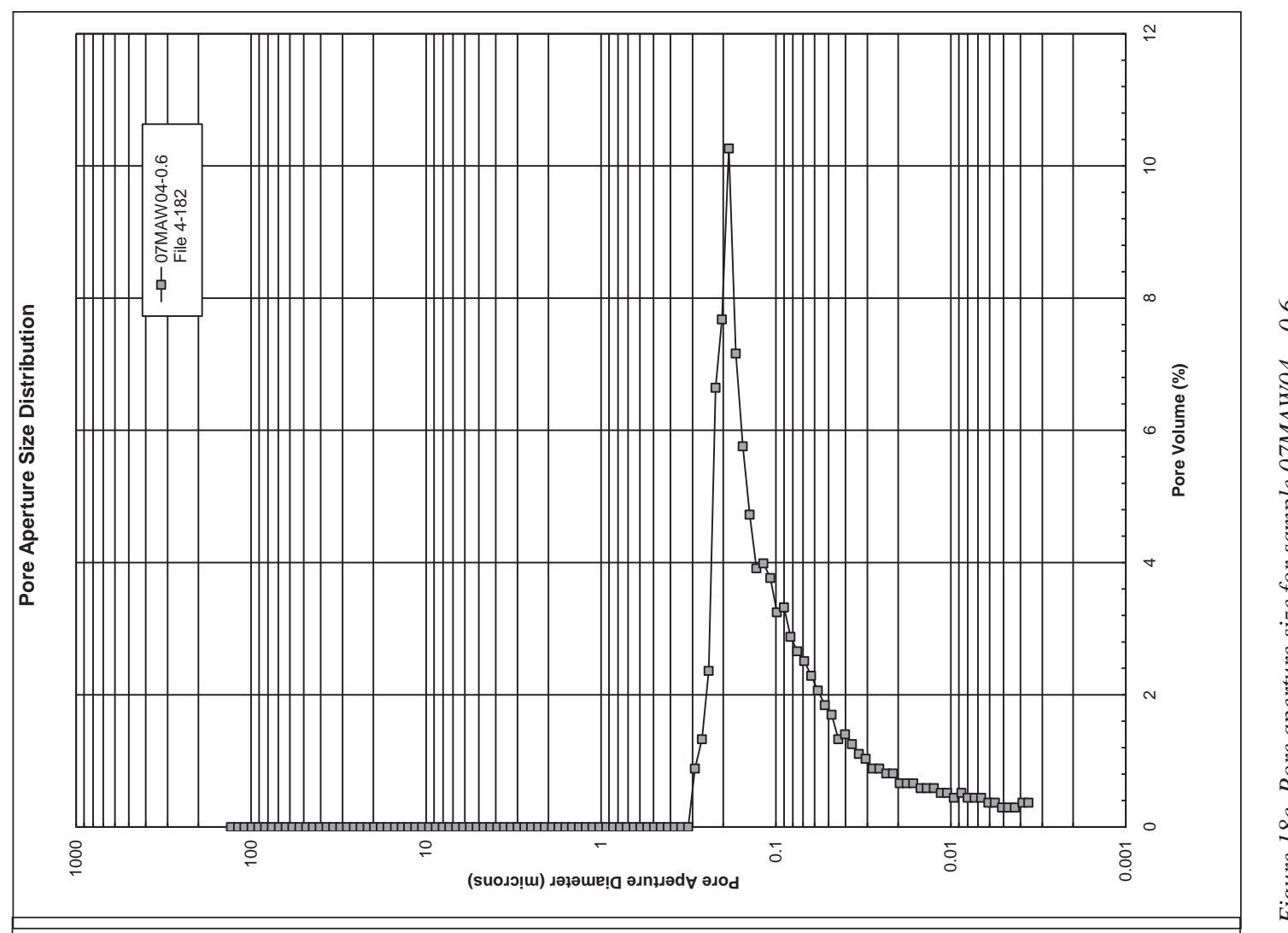

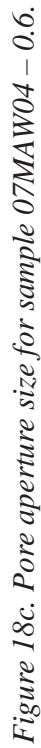

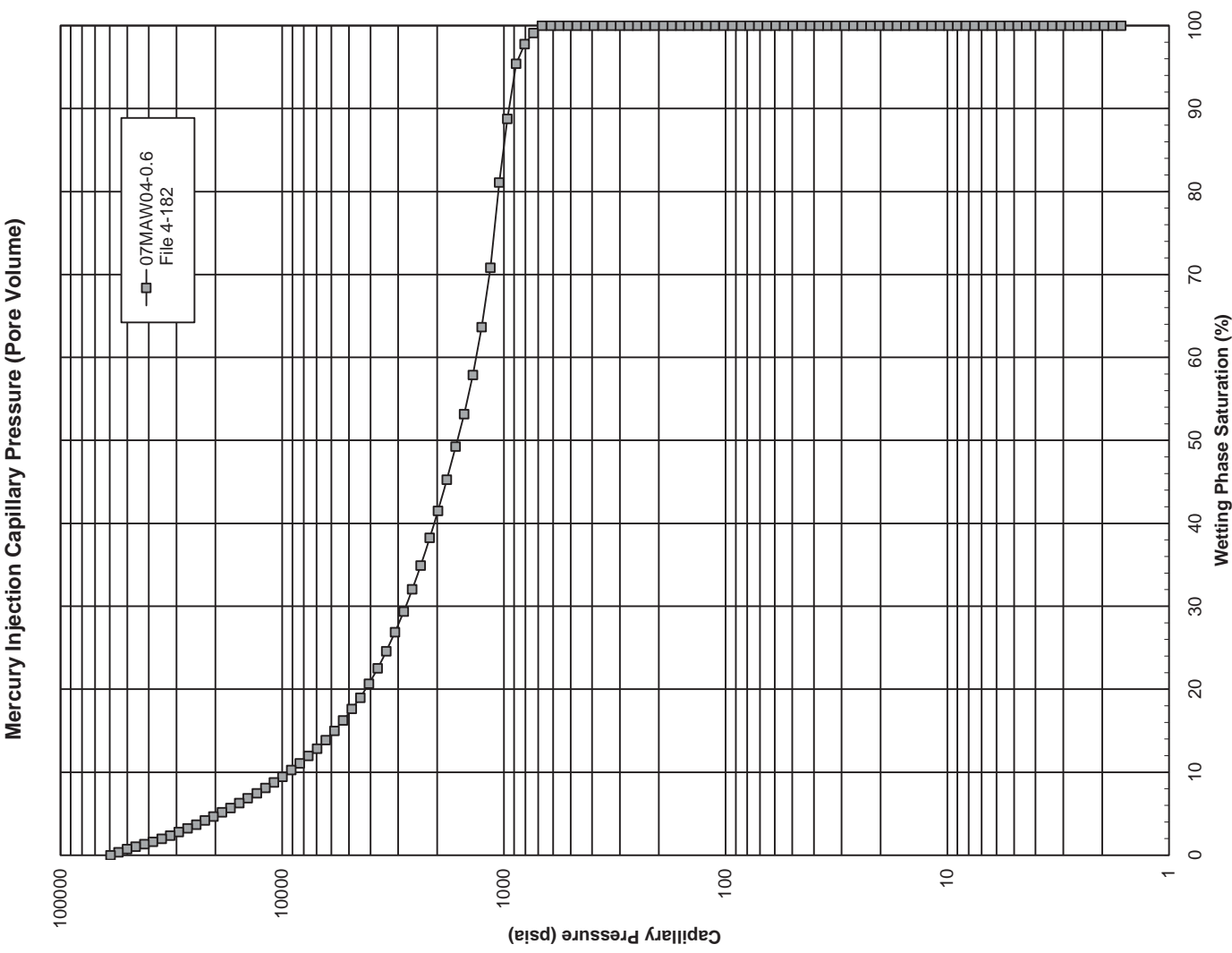

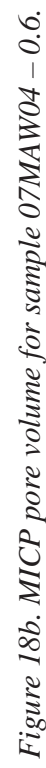



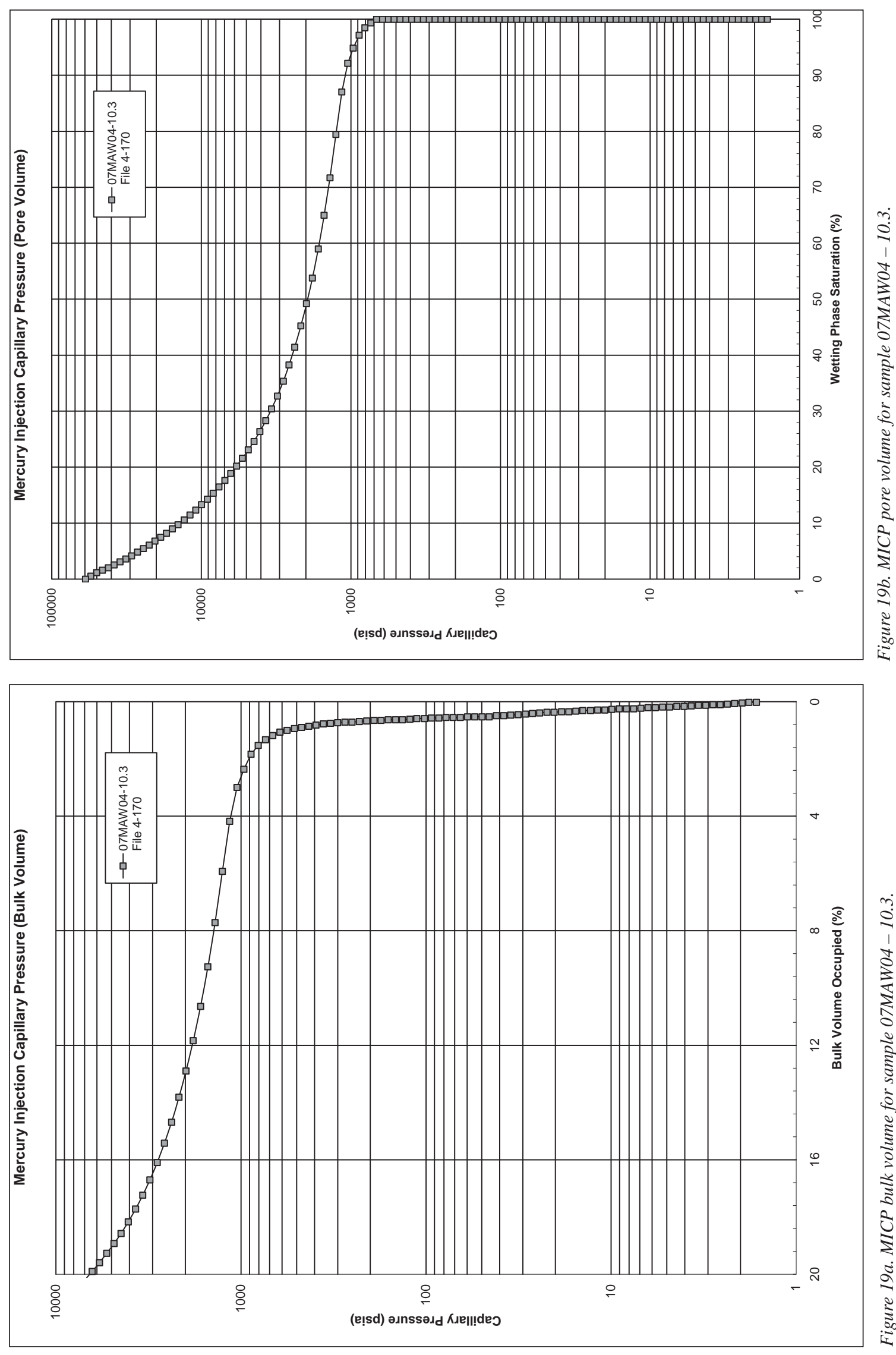

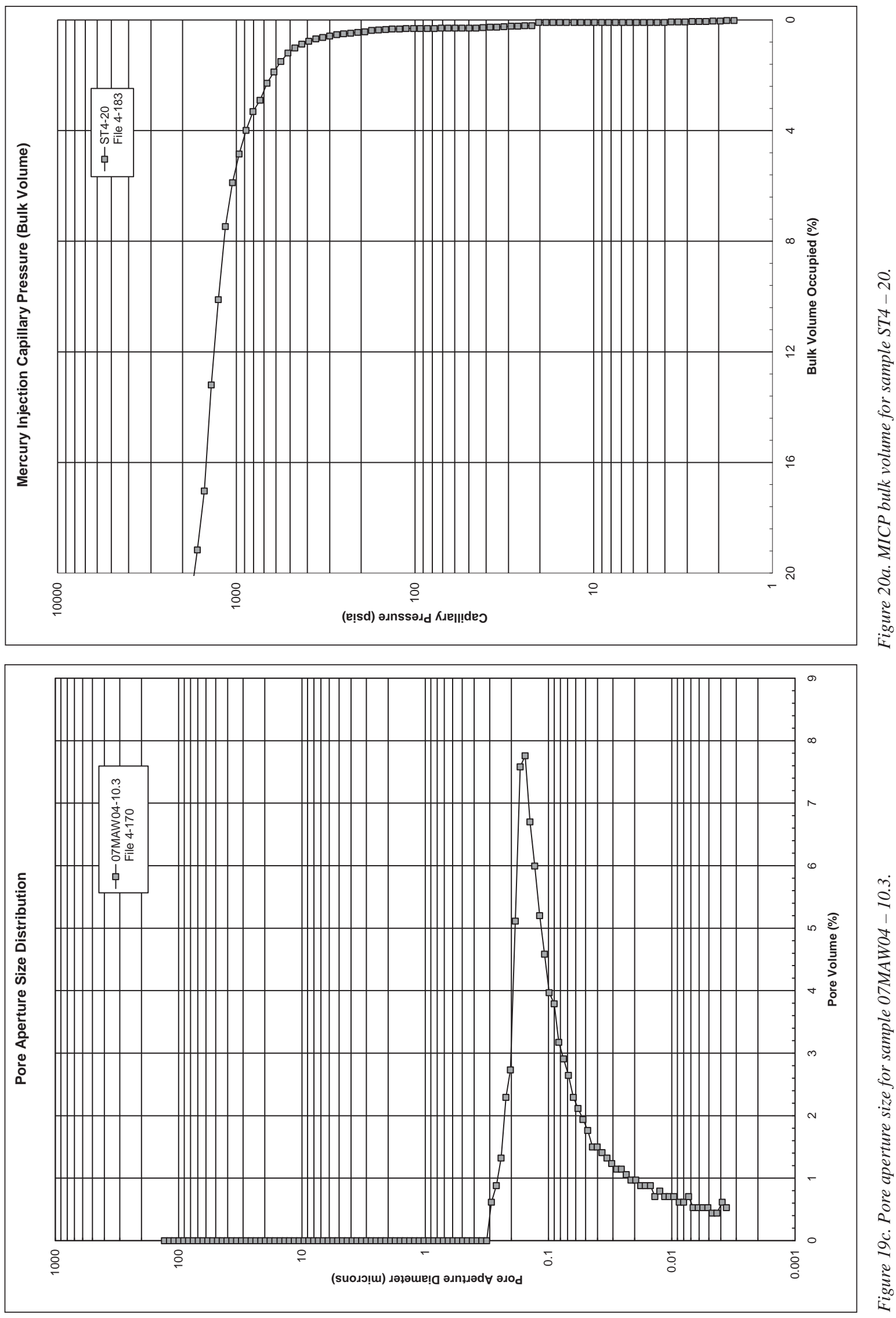

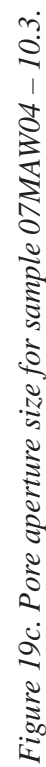



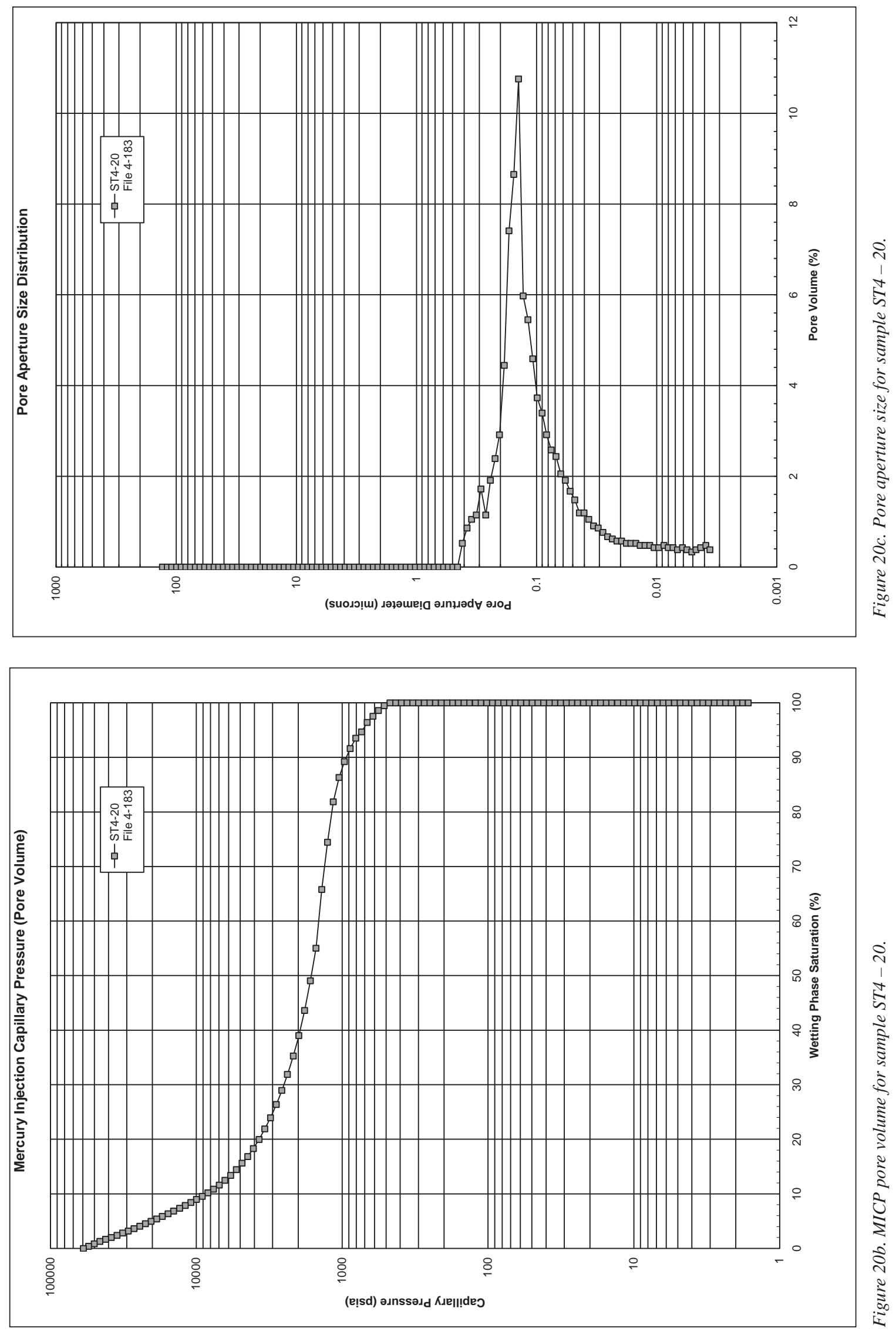


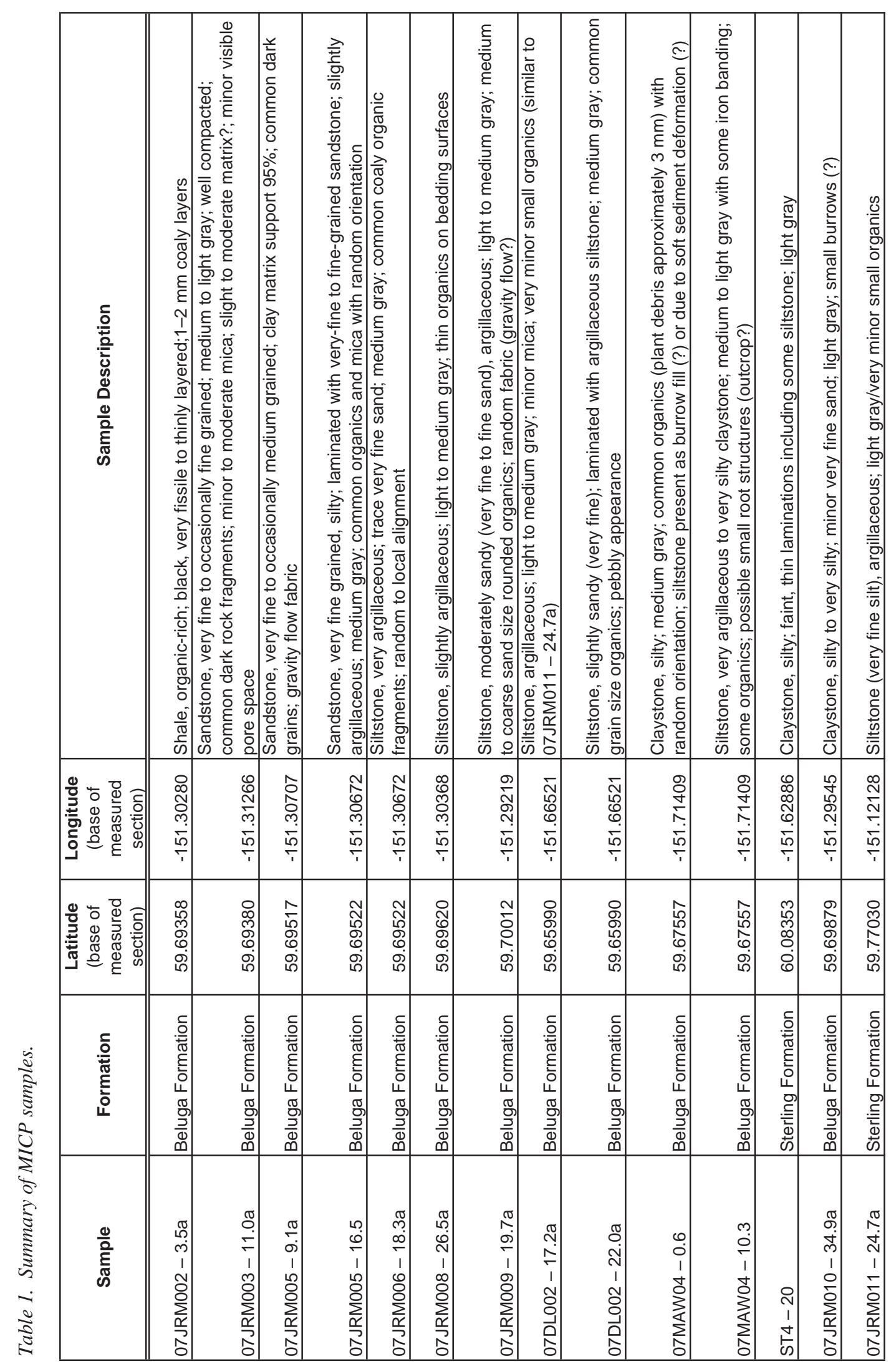


Table 2. Summary of pore system and capillary properties.

\begin{tabular}{|cccccccc}
\hline $\begin{array}{c}\text { Sample } \\
\text { Depth (ft) }\end{array}$ & $\begin{array}{c}\text { Porosity } \\
(\%)\end{array}$ & $\begin{array}{c}\text { Kair } \\
(\mathrm{md})\end{array}$ & $\begin{array}{c}\text { Median } \\
\text { Aperture }\end{array}$ & $\begin{array}{c}\text { apillary } \text { Pressure at Various Mercury Saturatior } \\
\text { entry }\end{array}$ & $5 \%$ & $7.5 \%$ & $10 \%$ \\
\hline \hline 07JRM002-3.5a & 11.3 & 0.0141 & 0.0299 & 964 & 1,500 & 1,735 & 1,962 \\
07JRM003-11.0a & 22.9 & 1.07 & 0.3717 & 78 & 124 & 138 & 150 \\
07JRM005-9.1a & 20.9 & 0.0856 & 0.1242 & 673 & 970 & 1,023 & 1,065 \\
07JRM005-16.5 & 22.1 & 0.494 & 0.3213 & 175 & 257 & 279 & 296 \\
07JRM006-18.3a & 22.1 & 0.0682 & 0.1013 & 881 & 1,172 & 1,243 & 1,291 \\
07JRM008-26.5a & 24.0 & 0.422 & 0.2900 & 300 & 400 & 411 & 423 \\
07JRM009-19.7a & 21.5 & 0.182 & 0.1960 & 430 & 577 & 600 & 621 \\
07JRM010-34.9a & 23.3 & 0.0901 & 0.1188 & 881 & 906 & 940 & 974 \\
07JRM011-24.7a & 34.0 & 0.150 & 0.1117 & 359 & 590 & 645 & 708 \\
07DL002-17.2a & 24.3 & 0.137 & 0.1452 & 229 & 381 & 428 & 470 \\
07DL002-22.0a & 25.0 & 0.171 & 0.1238 & 112 & 205 & 257 & 322 \\
07MAW04-0.6 & 26.6 & 0.134 & 0.1312 & 673 & 886 & 917 & 949 \\
07MAW04-10.3 & 23.1 & 0.0808 & 0.1095 & 673 & 960 & 1,040 & 1,092 \\
ST4-20 & 35.7 & 0.215 & 0.1308 & 470 & 725 & 845 & 936 \\
\hline \hline
\end{tabular}

Permeability calculated at maximum intrusion

Median aperture size is diameter in microns 


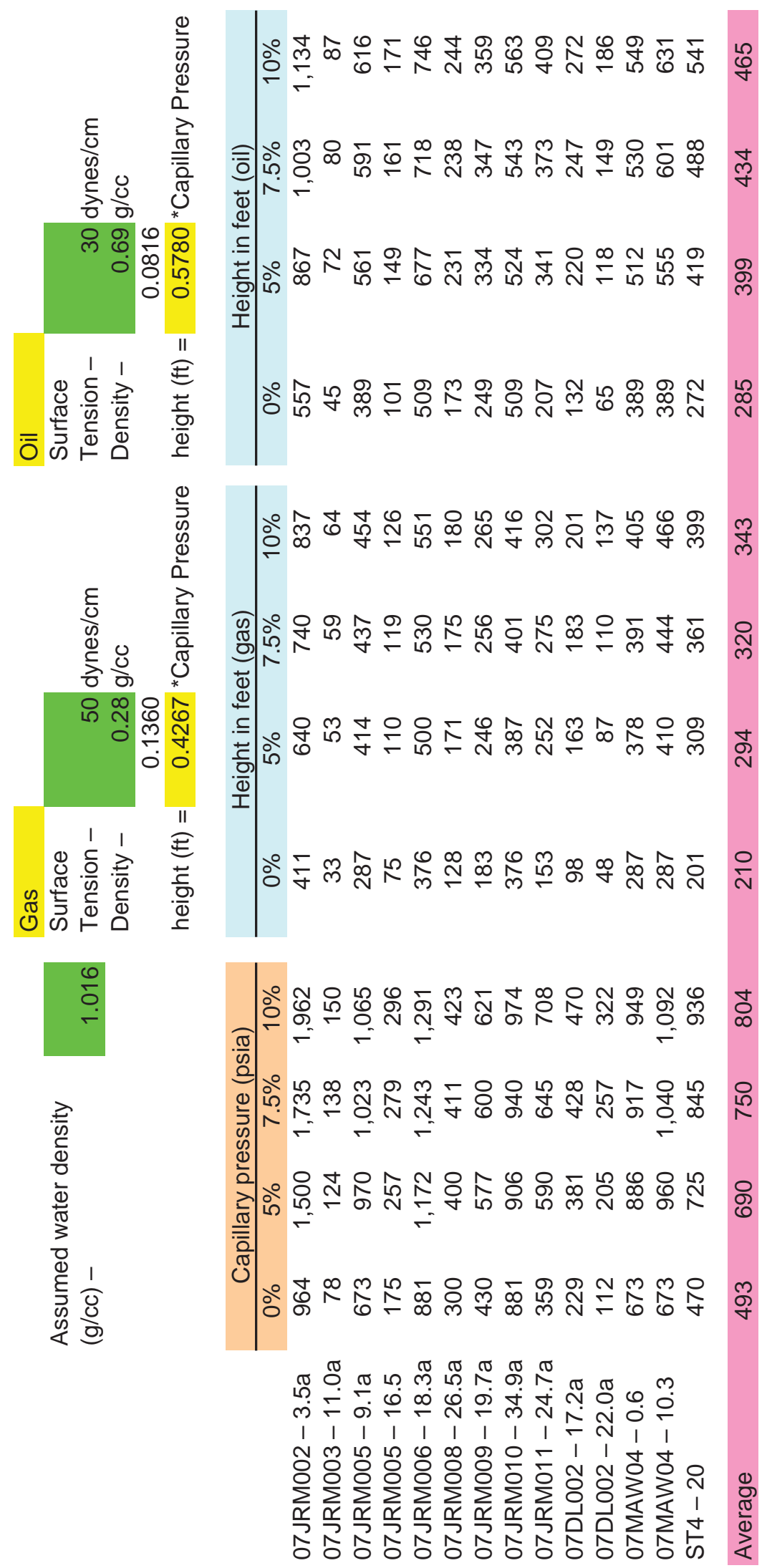


Table 4. MICP data for sample 07JRM002 - 3.5a.

\section{Mercury Injection Capillary Pressure 07JRM002 - 3.5a}

\begin{tabular}{|c|c|c|c|c|c|}
\hline & \multirow{2}{*}{\multicolumn{2}{|c|}{$\begin{array}{ll}\text { Porosity }= & 11.3 \% \text { (mercury) } \\
\text { Permeability }= & \mathrm{na} \\
\text { Median Pore Aperture }= & 0.0299 \mathrm{mi}\end{array}$}} & \\
\hline \multicolumn{3}{|c|}{ 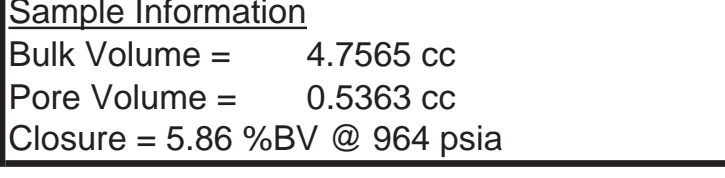 } & & & ns (diameter) \\
\hline $\begin{array}{l}\text { Capillary } \\
\text { Pressure } \\
\text { (psia) } \\
\end{array}$ & $\begin{array}{c}\text { Pore Aperture } \\
\text { Diameter } \\
\text { (microns) } \\
\end{array}$ & $\begin{array}{c}\text { Cumulative } \\
\text { Bulk Vol. (\%) } \\
\end{array}$ & $\begin{array}{l}\text { Wetting Phase } \\
\text { Saturation (\%) }\end{array}$ & $\begin{array}{l}\text { Incremental } \\
\text { Wetting Phase } \\
\text { Change (\%) } \\
\end{array}$ & $\begin{array}{l}\text { Saturation } \\
\text { Change } \\
\text { per psia } \\
\end{array}$ \\
\hline 1.64 & 130 & 0.09 & 100.0 & 0.00 & 0.0000 \\
\hline 1.80 & 119 & 0.14 & 100.0 & 0.00 & 0.0000 \\
\hline 1.96 & 109 & 0.20 & 100.0 & 0.00 & 0.0000 \\
\hline 2.15 & 99.2 & 0.26 & 100.0 & 0.00 & 0.0000 \\
\hline 2.35 & 90.8 & 0.34 & 100.0 & 0.00 & 0.0000 \\
\hline 2.57 & 83.0 & 0.49 & 100.0 & 0.00 & 0.0000 \\
\hline 2.81 & 75.9 & 0.63 & 100.0 & 0.00 & 0.0000 \\
\hline 3.08 & 69.3 & 0.76 & 100.0 & 0.00 & 0.0000 \\
\hline 3.37 & 63.3 & 0.85 & 100.0 & 0.00 & 0.0000 \\
\hline 3.68 & 58.0 & 1.08 & 100.0 & 0.00 & 0.0000 \\
\hline 4.03 & 52.9 & 1.23 & 100.0 & 0.00 & 0.0000 \\
\hline 4.41 & 48.4 & 1.33 & 100.0 & 0.00 & 0.0000 \\
\hline 4.82 & 44.3 & 1.47 & 100.0 & 0.00 & 0.0000 \\
\hline 5.27 & 40.5 & 1.54 & 100.0 & 0.00 & 0.0000 \\
\hline 5.77 & 37.0 & 1.63 & 100.0 & 0.00 & 0.0000 \\
\hline 6.31 & 33.8 & 1.79 & 100.0 & 0.00 & 0.0000 \\
\hline 6.90 & 30.9 & 1.93 & 100.0 & 0.00 & 0.0000 \\
\hline 7.55 & 28.3 & 2.04 & 100.0 & 0.00 & 0.0000 \\
\hline 8.26 & 25.8 & 2.17 & 100.0 & 0.00 & 0.0000 \\
\hline 9.04 & 23.6 & 2.28 & 100.0 & 0.00 & 0.0000 \\
\hline 9.89 & 21.6 & 2.36 & 100.0 & 0.00 & 0.0000 \\
\hline 10.8 & 19.8 & 2.45 & 100.0 & 0.00 & 0.0000 \\
\hline 11.8 & 18.1 & 2.53 & 100.0 & 0.00 & 0.0000 \\
\hline 12.9 & 16.5 & 2.62 & 100.0 & 0.00 & 0.0000 \\
\hline 14.2 & 15.0 & 2.71 & 100.0 & 0.00 & 0.0000 \\
\hline 15.5 & 13.8 & 2.81 & 100.0 & 0.00 & 0.0000 \\
\hline 16.9 & 12.6 & 2.93 & 100.0 & 0.00 & 0.0000 \\
\hline 18.5 & 11.5 & 3.13 & 100.0 & 0.00 & 0.0000 \\
\hline 20.3 & 10.5 & 3.24 & 100.0 & 0.00 & 0.0000 \\
\hline 22.2 & 9.61 & 3.33 & 100.0 & 0.00 & 0.0000 \\
\hline 24.3 & 8.78 & 3.41 & 100.0 & 0.00 & 0.0000 \\
\hline 26.6 & 8.02 & 3.52 & 100.0 & 0.00 & 0.0000 \\
\hline 29.0 & 7.36 & 3.62 & 100.0 & 0.00 & 0.0000 \\
\hline 31.8 & 6.71 & 3.72 & 100.0 & 0.00 & 0.0000 \\
\hline 34.8 & 6.13 & 3.81 & 100.0 & 0.00 & 0.0000 \\
\hline 38.0 & 5.61 & 3.92 & 100.0 & 0.00 & 0.0000 \\
\hline
\end{tabular}


Table 4 (cont.). MICP data for sample 07JRM002 - 3.5a.

\section{Mercury Injection Capillary Pressure 07JRM002 - 3.5a}

\begin{tabular}{|c|c|c|c|c|c|}
\hline $\begin{array}{c}\text { Capillary } \\
\text { Pressure } \\
\text { (psia) } \\
\end{array}$ & $\begin{array}{c}\text { Pore Aperture } \\
\text { Diameter } \\
\text { (microns) } \\
\end{array}$ & $\begin{array}{c}\text { Cumulative } \\
\text { Bulk Vol. (\%) } \\
\end{array}$ & $\begin{array}{l}\text { Wetting Phase } \\
\text { Saturation (\%) }\end{array}$ & $\begin{array}{c}\text { Incremental } \\
\text { Wetting Phase } \\
\text { Change (\%) } \\
\end{array}$ & $\begin{array}{c}\text { Saturation } \\
\text { Change } \\
\text { per psia }\end{array}$ \\
\hline 41.6 & 5.13 & 3.99 & 100.0 & 0.00 & 0.0000 \\
\hline 45.5 & 4.69 & 4.07 & 100.0 & 0.00 & 0.0000 \\
\hline 49.8 & 4.28 & 4.15 & 100.0 & 0.00 & 0.0000 \\
\hline 54.5 & 3.91 & 4.23 & 100.0 & 0.00 & 0.0000 \\
\hline 59.6 & 3.58 & 4.27 & 100.0 & 0.00 & 0.0000 \\
\hline 65.2 & 3.27 & 4.35 & 100.0 & 0.00 & 0.0000 \\
\hline 71.3 & 2.99 & 4.41 & 100.0 & 0.00 & 0.0000 \\
\hline 78.0 & 2.73 & 4.44 & 100.0 & 0.00 & 0.0000 \\
\hline 85.3 & 2.50 & 4.49 & 100.0 & 0.00 & 0.0000 \\
\hline 93.4 & 2.28 & 4.55 & 100.0 & 0.00 & 0.0000 \\
\hline 102 & 2.09 & 4.60 & 100.0 & 0.00 & 0.0000 \\
\hline 112 & 1.90 & 4.67 & 100.0 & 0.00 & 0.0000 \\
\hline 122 & 1.75 & 4.70 & 100.0 & 0.00 & 0.0000 \\
\hline 134 & 1.59 & 4.75 & 100.0 & 0.00 & 0.0000 \\
\hline 146 & 1.46 & 4.78 & 100.0 & 0.00 & 0.0000 \\
\hline 160 & 1.33 & 4.83 & 100.0 & 0.00 & 0.0000 \\
\hline 175 & 1.22 & 4.87 & 100.0 & 0.00 & 0.0000 \\
\hline 191 & 1.12 & 4.92 & 100.0 & 0.00 & 0.0000 \\
\hline 209 & 1.02 & 4.97 & 100.0 & 0.00 & 0.0000 \\
\hline 229 & 0.932 & 5.00 & 100.0 & 0.00 & 0.0000 \\
\hline 251 & 0.850 & 5.04 & 100.0 & 0.00 & 0.0000 \\
\hline 274 & 0.779 & 5.09 & 100.0 & 0.00 & 0.0000 \\
\hline 300 & 0.711 & 5.14 & 100.0 & 0.00 & 0.0000 \\
\hline 328 & 0.650 & 5.18 & 100.0 & 0.00 & 0.0000 \\
\hline 359 & 0.594 & 5.23 & 100.0 & 0.00 & 0.0000 \\
\hline 393 & 0.543 & 5.26 & 100.0 & 0.00 & 0.0000 \\
\hline 430 & 0.496 & 5.31 & 100.0 & 0.00 & 0.0000 \\
\hline 470 & 0.454 & 5.35 & 100.0 & 0.00 & 0.0000 \\
\hline 514 & 0.415 & 5.38 & 100.0 & 0.00 & 0.0000 \\
\hline 563 & 0.379 & 5.46 & 100.0 & 0.00 & 0.0000 \\
\hline 615 & 0.347 & 5.52 & 100.0 & 0.00 & 0.0000 \\
\hline 673 & 0.317 & 5.57 & 100.0 & 0.00 & 0.0000 \\
\hline 736 & 0.290 & 5.63 & 100.0 & 0.00 & 0.0000 \\
\hline 806 & 0.265 & 5.69 & 100.0 & 0.00 & 0.0000 \\
\hline 881 & 0.242 & 5.77 & 100.0 & 0.00 & 0.0000 \\
\hline 964 & 0.221 & 5.86 & 100.0 & 0.00 & 0.0000 \\
\hline 1050 & 0.203 & 5.94 & 99.3 & 0.69 & 0.0080 \\
\hline 1150 & 0.186 & 6.05 & 98.3 & 0.96 & 0.0096 \\
\hline 1260 & 0.169 & 6.15 & 97.4 & 0.96 & 0.0088 \\
\hline 1380 & 0.155 & 6.28 & 96.3 & 1.10 & 0.0092 \\
\hline 1510 & 0.141 & 6.43 & 94.9 & 1.38 & 0.0106 \\
\hline 1650 & 0.129 & 6.60 & 93.4 & 1.51 & 0.0108 \\
\hline
\end{tabular}


Table 4 (cont.). MICP data for sample 07JRM002 - 3.5a.

\section{Mercury Injection Capillary Pressure 07JRM002 - 3.5a}

\begin{tabular}{|c|c|c|c|c|c|}
\hline $\begin{array}{c}\text { Capillary } \\
\text { Pressure } \\
\text { (psia) } \\
\end{array}$ & $\begin{array}{c}\text { Pore Aperture } \\
\text { Diameter } \\
\text { (microns) } \\
\end{array}$ & $\begin{array}{c}\text { Cumulative } \\
\text { Bulk Vol. (\%) }\end{array}$ & $\begin{array}{l}\text { Wetting Phase } \\
\text { Saturation (\%) }\end{array}$ & $\begin{array}{c}\text { Incremental } \\
\text { Wetting Phase } \\
\text { Change (\%) }\end{array}$ & $\begin{array}{c}\text { Saturation } \\
\text { Change } \\
\text { per psia } \\
\end{array}$ \\
\hline 1810 & 0.1179 & 6.79 & 91.7 & 1.65 & 0.0103 \\
\hline 1980 & 0.1077 & 7.00 & 89.8 & 1.93 & 0.0113 \\
\hline 2160 & 0.0988 & 7.23 & 87.8 & 2.06 & 0.0115 \\
\hline 2370 & 0.0900 & 7.51 & 85.3 & 2.48 & 0.0118 \\
\hline 2590 & 0.0824 & 7.79 & 82.8 & 2.48 & 0.0113 \\
\hline 2830 & 0.0754 & 8.10 & 80.1 & 2.75 & 0.0115 \\
\hline 3100 & 0.0688 & 8.44 & 77.0 & 3.03 & 0.0112 \\
\hline 3390 & 0.0629 & 8.76 & 74.1 & 2.89 & 0.0100 \\
\hline 3710 & 0.0575 & 9.12 & 71.0 & 3.16 & 0.0099 \\
\hline 4060 & 0.0525 & 9.47 & 67.8 & 3.16 & 0.0090 \\
\hline 4440 & 0.0480 & 9.81 & 64.8 & 3.03 & 0.0080 \\
\hline 4850 & 0.0440 & 10.13 & 61.9 & 2.89 & 0.0070 \\
\hline 5310 & 0.0402 & 10.46 & 59.0 & 2.89 & 0.0063 \\
\hline 5810 & 0.0367 & 10.78 & 56.1 & 2.89 & 0.0058 \\
\hline 6360 & 0.0335 & 11.09 & 53.4 & 2.75 & 0.0050 \\
\hline 6950 & 0.0307 & 11.38 & 50.8 & 2.61 & 0.0044 \\
\hline 7610 & 0.0280 & 11.68 & 48.1 & 2.61 & 0.0040 \\
\hline 8320 & 0.0256 & 11.94 & 45.8 & 2.34 & 0.0033 \\
\hline 9100 & 0.0234 & 12.18 & 43.6 & 2.20 & 0.0028 \\
\hline 9960 & 0.0214 & 12.43 & 41.4 & 2.20 & 0.0026 \\
\hline 10900 & 0.0196 & 12.65 & 39.5 & 1.93 & 0.0020 \\
\hline 11900 & 0.0179 & 12.86 & 37.6 & 1.93 & 0.0019 \\
\hline 13000 & 0.0164 & 13.08 & 35.6 & 1.93 & 0.0018 \\
\hline 14300 & 0.0149 & 13.29 & 33.7 & 1.93 & 0.0015 \\
\hline 15600 & 0.0137 & 13.50 & 31.9 & 1.79 & 0.0014 \\
\hline 17100 & 0.0125 & 13.70 & 30.1 & 1.79 & 0.0012 \\
\hline 18700 & 0.0114 & 13.90 & 28.3 & 1.79 & 0.0011 \\
\hline 20400 & 0.0105 & 14.08 & 26.7 & 1.65 & 0.0010 \\
\hline 22300 & 0.0096 & 14.28 & 24.9 & 1.79 & 0.0009 \\
\hline 24400 & 0.0087 & 14.50 & 23.0 & 1.93 & 0.0009 \\
\hline 26700 & 0.0080 & 14.71 & 21.0 & 1.93 & 0.0008 \\
\hline 29300 & 0.0073 & 14.93 & 19.1 & 1.93 & 0.0007 \\
\hline 32000 & 0.0067 & 15.15 & 17.2 & 1.93 & 0.0007 \\
\hline 35000 & 0.0061 & 15.38 & 15.1 & 2.06 & 0.0007 \\
\hline 38300 & 0.0056 & 15.62 & 12.9 & 2.20 & 0.0007 \\
\hline 41900 & 0.0051 & 15.87 & 10.7 & 2.20 & 0.0006 \\
\hline 45800 & 0.0047 & 16.13 & 8.4 & 2.34 & 0.0006 \\
\hline 50100 & 0.0043 & 16.46 & 5.5 & 2.89 & 0.0007 \\
\hline 54800 & 0.0039 & 16.76 & 2.8 & 2.75 & 0.0006 \\
\hline 59500 & 0.0036 & 17.07 & 0.0 & 2.75 & 0.0006 \\
\hline
\end{tabular}


Table 5. MICP data for sample 07JRM003 - 11.0a.

\section{Mercury Injection Capillary Pressure 07JRM003 - 11.0a}

\begin{tabular}{|c|c|c|c|c|c|}
\hline $\begin{array}{l}\text { Sample Information } \\
\text { Bulk Volume }= \\
\text { Pore Volume }= \\
\text { Closure }=0.97 \% \mathrm{BV}\end{array}$ & $\begin{array}{l}4.8422 \text { cc } \\
1.1077 \text { cc } \\
0 \text { psia }\end{array}$ & & $\begin{array}{l}\text { Porosity }= \\
\text { Permeability = } \\
\text { Median Pore Apertu }\end{array}$ & $\begin{array}{l}22.9 \% \text { (mercury) } \\
72 \mathrm{md} \text { (mercury } \\
.3717 \text { microns }\end{array}$ & meter) \\
\hline $\begin{array}{l}\text { Capillary } \\
\text { Pressure } \\
\text { (psia) } \\
\end{array}$ & $\begin{array}{l}\text { Pore Aperture } \\
\text { Diameter } \\
\text { (microns) }\end{array}$ & $\begin{array}{l}\text { Cumulative } \\
\text { Bulk Vol. (\%) }\end{array}$ & $\begin{array}{l}\text { Wetting Phase } \\
\text { Saturation (\%) }\end{array}$ & $\begin{array}{l}\text { Incremental } \\
\text { Wetting Phase } \\
\text { Change (\%) }\end{array}$ & $\begin{array}{c}\text { Saturation } \\
\text { Change } \\
\text { per psia }\end{array}$ \\
\hline 1.64 & 130 & 0.02 & 100.0 & 0.00 & 0.0000 \\
\hline 1.80 & 119 & 0.02 & 100.0 & 0.00 & 0.0000 \\
\hline 1.96 & 109 & 0.04 & 100.0 & 0.00 & 0.0000 \\
\hline 2.15 & 99.2 & 0.06 & 100.0 & 0.00 & 0.0000 \\
\hline 2.35 & 90.8 & 0.06 & 100.0 & 0.00 & 0.0000 \\
\hline 2.57 & 83.0 & 0.08 & 100.0 & 0.00 & 0.0000 \\
\hline 2.81 & 75.9 & 0.08 & 100.0 & 0.00 & 0.0000 \\
\hline 3.08 & 69.3 & 0.10 & 100.0 & 0.00 & 0.0000 \\
\hline 3.37 & 63.3 & 0.10 & 100.0 & 0.00 & 0.0000 \\
\hline 3.68 & 58.0 & 0.12 & 100.0 & 0.00 & 0.0000 \\
\hline 4.03 & 52.9 & 0.12 & 100.0 & 0.00 & 0.0000 \\
\hline 4.41 & 48.4 & 0.14 & 100.0 & 0.00 & 0.0000 \\
\hline 4.82 & 44.3 & 0.14 & 100.0 & 0.00 & 0.0000 \\
\hline 5.27 & 40.5 & 0.14 & 100.0 & 0.00 & 0.0000 \\
\hline 5.77 & 37.0 & 0.17 & 100.0 & 0.00 & 0.0000 \\
\hline 6.31 & 33.8 & 0.17 & 100.0 & 0.00 & 0.0000 \\
\hline 6.90 & 30.9 & 0.19 & 100.0 & 0.00 & 0.0000 \\
\hline 7.55 & 28.3 & 0.19 & 100.0 & 0.00 & 0.0000 \\
\hline 8.26 & 25.8 & 0.21 & 100.0 & 0.00 & 0.0000 \\
\hline 9.04 & 23.6 & 0.21 & 100.0 & 0.00 & 0.0000 \\
\hline 9.89 & 21.6 & 0.23 & 100.0 & 0.00 & 0.0000 \\
\hline 10.8 & 19.8 & 0.23 & 100.0 & 0.00 & 0.0000 \\
\hline 11.8 & 18.1 & 0.25 & 100.0 & 0.00 & 0.0000 \\
\hline 12.9 & 16.5 & 0.27 & 100.0 & 0.00 & 0.0000 \\
\hline 14.2 & 15.0 & 0.29 & 100.0 & 0.00 & 0.0000 \\
\hline 15.5 & 13.8 & 0.31 & 100.0 & 0.00 & 0.0000 \\
\hline 16.9 & 12.6 & 0.33 & 100.0 & 0.00 & 0.0000 \\
\hline 18.5 & 11.5 & 0.35 & 100.0 & 0.00 & 0.0000 \\
\hline 20.3 & 10.5 & 0.37 & 100.0 & 0.00 & 0.0000 \\
\hline 22.2 & 9.61 & 0.39 & 100.0 & 0.00 & 0.0000 \\
\hline 24.3 & 8.78 & 0.43 & 100.0 & 0.00 & 0.0000 \\
\hline 26.6 & 8.02 & 0.45 & 100.0 & 0.00 & 0.0000 \\
\hline 29.0 & 7.36 & 0.50 & 100.0 & 0.00 & 0.0000 \\
\hline 31.8 & 6.71 & 0.52 & 100.0 & 0.00 & 0.0000 \\
\hline 34.8 & 6.13 & 0.56 & 100.0 & 0.00 & 0.0000 \\
\hline 38.0 & 5.61 & 0.60 & 100.0 & 0.00 & 0.0000 \\
\hline
\end{tabular}


Table 5 (cont.). MICP data for sample 07JRM003 - 11.0a.

\section{Mercury Injection Capillary Pressure 07JRM003 - 11.0a}

\begin{tabular}{|c|c|c|c|c|c|}
\hline $\begin{array}{c}\text { Capillary } \\
\text { Pressure } \\
\text { (psia) } \\
\end{array}$ & $\begin{array}{c}\text { Pore Aperture } \\
\text { Diameter } \\
\text { (microns) } \\
\end{array}$ & $\begin{array}{c}\text { Cumulative } \\
\text { Bulk Vol. (\%) } \\
\end{array}$ & $\begin{array}{l}\text { Wetting Phase } \\
\text { Saturation (\%) }\end{array}$ & $\begin{array}{c}\text { Incremental } \\
\text { Wetting Phase } \\
\text { Change (\%) } \\
\end{array}$ & $\begin{array}{c}\text { Saturation } \\
\text { Change } \\
\text { per psia } \\
\end{array}$ \\
\hline 41.6 & 5.13 & 0.64 & 100.0 & 0.00 & 0.0000 \\
\hline 45.5 & 4.69 & 0.68 & 100.0 & 0.00 & 0.0000 \\
\hline 49.8 & 4.28 & 0.72 & 100.0 & 0.00 & 0.0000 \\
\hline 54.5 & 3.91 & 0.74 & 100.0 & 0.00 & 0.0000 \\
\hline 59.6 & 3.58 & 0.78 & 100.0 & 0.00 & 0.0000 \\
\hline 65.2 & 3.27 & 0.83 & 100.0 & 0.00 & 0.0000 \\
\hline 71.3 & 2.99 & 0.89 & 100.0 & 0.00 & 0.0000 \\
\hline 78.0 & 2.73 & 0.97 & 100.0 & 0.00 & 0.0000 \\
\hline 85.3 & 2.50 & 1.05 & 99.6 & 0.36 & 0.0495 \\
\hline 93.4 & 2.28 & 1.20 & 99.0 & 0.63 & 0.0781 \\
\hline 102 & 2.09 & 1.38 & 98.2 & 0.81 & 0.0945 \\
\hline 112 & 1.90 & 1.75 & 96.6 & 1.63 & 0.1626 \\
\hline 122 & 1.75 & 2.02 & 95.4 & 1.17 & 0.1174 \\
\hline 134 & 1.59 & 2.50 & 93.3 & 2.08 & 0.1731 \\
\hline 146 & 1.46 & 3.03 & 91.0 & 2.35 & 0.1957 \\
\hline 160 & 1.33 & 3.74 & 87.9 & 3.07 & 0.2194 \\
\hline 175 & 1.22 & 4.48 & 84.6 & 3.25 & 0.2168 \\
\hline 191 & 1.12 & 5.29 & 81.1 & 3.52 & 0.2202 \\
\hline 209 & 1.02 & 6.07 & 77.7 & 3.43 & 0.1907 \\
\hline 229 & 0.932 & 6.85 & 74.3 & 3.43 & 0.1716 \\
\hline 251 & 0.850 & 7.58 & 71.1 & 3.16 & 0.1437 \\
\hline 274 & 0.779 & 8.18 & 68.5 & 2.62 & 0.1139 \\
\hline 300 & 0.711 & 8.67 & 66.3 & 2.17 & 0.0834 \\
\hline 328 & 0.650 & 9.21 & 64.0 & 2.35 & 0.0839 \\
\hline 359 & 0.594 & 9.79 & 61.4 & 2.53 & 0.0816 \\
\hline 393 & 0.543 & 10.32 & 59.1 & 2.35 & 0.0691 \\
\hline 430 & 0.496 & 10.86 & 56.7 & 2.35 & 0.0635 \\
\hline 470 & 0.454 & 11.36 & 54.6 & 2.17 & 0.0542 \\
\hline 514 & 0.415 & 11.87 & 52.3 & 2.26 & 0.0513 \\
\hline 563 & 0.379 & 12.28 & 50.5 & 1.81 & 0.0369 \\
\hline 615 & 0.347 & 12.78 & 48.3 & 2.17 & 0.0417 \\
\hline 673 & 0.317 & 13.21 & 46.4 & 1.90 & 0.0327 \\
\hline 736 & 0.290 & 13.67 & 44.4 & 1.99 & 0.0315 \\
\hline 806 & 0.265 & 14.10 & 42.5 & 1.90 & 0.0271 \\
\hline 881 & 0.242 & 14.49 & 40.8 & 1.72 & 0.0229 \\
\hline 964 & 0.221 & 14.93 & 38.9 & 1.90 & 0.0229 \\
\hline 1050 & 0.203 & 15.32 & 37.2 & 1.72 & 0.0200 \\
\hline 1150 & 0.186 & 15.69 & 35.6 & 1.63 & 0.0163 \\
\hline 1260 & 0.169 & 16.04 & 34.1 & 1.54 & 0.0140 \\
\hline 1380 & 0.155 & 16.39 & 32.5 & 1.54 & 0.0128 \\
\hline 1510 & 0.141 & 16.74 & 31.0 & 1.54 & 0.0118 \\
\hline 1650 & 0.129 & 17.07 & 29.5 & 1.45 & 0.0103 \\
\hline
\end{tabular}


Table 5 (cont.). MICP data for sample 07JRM003 - 11.0a.

\section{Mercury Injection Capillary Pressure 07JRM003 - 11.0a}

\begin{tabular}{|c|c|c|c|c|c|}
\hline $\begin{array}{l}\text { Capillary } \\
\text { Pressure } \\
\text { (psia) } \\
\end{array}$ & $\begin{array}{c}\text { Pore Aperture } \\
\text { Diameter } \\
\text { (microns) } \\
\end{array}$ & $\begin{array}{c}\text { Cumulative } \\
\text { Bulk Vol. (\%) } \\
\end{array}$ & $\begin{array}{l}\text { Wetting Phase } \\
\text { Saturation (\%) }\end{array}$ & $\begin{array}{c}\text { Incremental } \\
\text { Wetting Phase } \\
\text { Change (\%) } \\
\end{array}$ & $\begin{array}{c}\text { Saturation } \\
\text { Change } \\
\text { per psia } \\
\end{array}$ \\
\hline 1810 & 0.1179 & 17.40 & 28.1 & 1.45 & 0.0090 \\
\hline 1980 & 0.1077 & 17.69 & 26.8 & 1.26 & 0.0074 \\
\hline 2160 & 0.0988 & 17.98 & 25.6 & 1.26 & 0.0070 \\
\hline 2370 & 0.0900 & 18.27 & 24.3 & 1.26 & 0.0060 \\
\hline 2590 & 0.0824 & 18.56 & 23.0 & 1.26 & 0.0057 \\
\hline 2830 & 0.0754 & 18.81 & 22.0 & 1.08 & 0.0045 \\
\hline 3100 & 0.0688 & 19.06 & 20.9 & 1.08 & 0.0040 \\
\hline 3390 & 0.0629 & 19.30 & 19.8 & 1.08 & 0.0037 \\
\hline 3710 & 0.0575 & 19.53 & 18.8 & 0.99 & 0.0031 \\
\hline 4060 & 0.0525 & 19.76 & 17.8 & 0.99 & 0.0028 \\
\hline 4440 & 0.0480 & 19.96 & 16.9 & 0.90 & 0.0024 \\
\hline 4850 & 0.0440 & 20.17 & 16.0 & 0.90 & 0.0022 \\
\hline 5310 & 0.0402 & 20.36 & 15.2 & 0.81 & 0.0018 \\
\hline 5810 & 0.0367 & 20.54 & 14.4 & 0.81 & 0.0016 \\
\hline 6360 & 0.0335 & 20.73 & 13.6 & 0.81 & 0.0015 \\
\hline 6950 & 0.0307 & 20.91 & 12.7 & 0.81 & 0.0014 \\
\hline 7610 & 0.0280 & 21.08 & 12.0 & 0.72 & 0.0011 \\
\hline 8320 & 0.0256 & 21.24 & 11.3 & 0.72 & 0.0010 \\
\hline 9100 & 0.0234 & 21.41 & 10.6 & 0.72 & 0.0009 \\
\hline 9960 & 0.0214 & 21.57 & 9.8 & 0.72 & 0.0008 \\
\hline 10900 & 0.0196 & 21.72 & 9.2 & 0.63 & 0.0007 \\
\hline 11900 & 0.0179 & 21.86 & 8.6 & 0.63 & 0.0006 \\
\hline 13000 & 0.0164 & 22.01 & 7.9 & 0.63 & 0.0006 \\
\hline 14300 & 0.0149 & 22.15 & 7.3 & 0.63 & 0.0005 \\
\hline 15600 & 0.0137 & 22.30 & 6.7 & 0.63 & 0.0005 \\
\hline 17100 & 0.0125 & 22.42 & 6.1 & 0.54 & 0.0004 \\
\hline 18700 & 0.0114 & 22.54 & 5.6 & 0.54 & 0.0003 \\
\hline 20400 & 0.0105 & 22.67 & 5.1 & 0.54 & 0.0003 \\
\hline 22300 & 0.0096 & 22.79 & 4.5 & 0.54 & 0.0003 \\
\hline 24400 & 0.0087 & 22.92 & 4.0 & 0.54 & 0.0003 \\
\hline 26700 & 0.0080 & 23.02 & 3.5 & 0.45 & 0.0002 \\
\hline 29300 & 0.0073 & 23.12 & 3.1 & 0.45 & 0.0002 \\
\hline 32000 & 0.0067 & 23.23 & 2.6 & 0.45 & 0.0002 \\
\hline 35000 & 0.0061 & 23.33 & 2.2 & 0.45 & 0.0002 \\
\hline 38300 & 0.0056 & 23.41 & 1.8 & 0.36 & 0.0001 \\
\hline 41900 & 0.0051 & 23.49 & 1.4 & 0.36 & 0.0001 \\
\hline 45800 & 0.0047 & 23.58 & 1.1 & 0.36 & 0.0001 \\
\hline 50100 & 0.0043 & 23.64 & 0.8 & 0.27 & 0.0001 \\
\hline 54800 & 0.0039 & 23.70 & 0.5 & 0.27 & 0.0001 \\
\hline 59500 & 0.0036 & 23.82 & 0.0 & 0.54 & 0.0001 \\
\hline
\end{tabular}


Table 6. MICP data for sample 07JRM005 - 9.1a.

\section{Mercury Injection Capillary Pressure 07JRM005 - 9.1a}

\begin{tabular}{|c|c|c|c|c|c|}
\hline \multicolumn{3}{|c|}{$\begin{array}{ll}\text { Sample Information } & \\
\text { Bulk Volume }= & 8.2263 \mathrm{cc} \\
\text { Pore Volume }= & 1.7183 \mathrm{cc} \\
\text { Closure }=1.17 \% \text { BV @ } 317 \mathrm{psia} \\
\end{array}$} & \multicolumn{3}{|c|}{$\begin{array}{lc}\text { Porosity }= & 20.9 \% \text { (mercury) } \\
\text { Permeability }= & 0.0856 \text { md (mercury) } \\
\text { Median Pore Aperture }=0.1242 \text { microns (diameter) }\end{array}$} \\
\hline $\begin{array}{l}\text { Capillary } \\
\text { Pressure } \\
\text { (psia) } \\
\end{array}$ & $\begin{array}{l}\text { Pore Aperture } \\
\text { Diameter } \\
\text { (microns) } \\
\end{array}$ & $\begin{array}{l}\text { Cumulative } \\
\text { Bulk Vol. (\%) } \\
\end{array}$ & $\begin{array}{l}\text { Wetting Phase } \\
\text { Saturation (\%) }\end{array}$ & $\begin{array}{l}\text { Incremental } \\
\text { Wetting Phase } \\
\text { Change (\%) } \\
\end{array}$ & $\begin{array}{l}\text { Saturation } \\
\text { Change } \\
\text { per psia } \\
\end{array}$ \\
\hline 1.64 & 130 & 0.02 & 100.0 & 0.00 & 0.0000 \\
\hline 1.80 & 119 & 0.02 & 100.0 & 0.00 & 0.0000 \\
\hline 1.96 & 109 & 0.04 & 100.0 & 0.00 & 0.0000 \\
\hline 2.15 & 99.2 & 0.04 & 100.0 & 0.00 & 0.0000 \\
\hline 2.35 & 90.8 & 0.06 & 100.0 & 0.00 & 0.0000 \\
\hline 2.57 & 83.0 & 0.06 & 100.0 & 0.00 & 0.0000 \\
\hline 2.81 & 75.9 & 0.06 & 100.0 & 0.00 & 0.0000 \\
\hline 3.08 & 69.3 & 0.09 & 100.0 & 0.00 & 0.0000 \\
\hline 3.37 & 63.3 & 0.09 & 100.0 & 0.00 & 0.0000 \\
\hline 3.68 & 58.0 & 0.09 & 100.0 & 0.00 & 0.0000 \\
\hline 4.03 & 52.9 & 0.09 & 100.0 & 0.00 & 0.0000 \\
\hline 4.41 & 48.4 & 0.09 & 100.0 & 0.00 & 0.0000 \\
\hline 4.82 & 44.3 & 0.09 & 100.0 & 0.00 & 0.0000 \\
\hline 5.27 & 40.5 & 0.13 & 100.0 & 0.00 & 0.0000 \\
\hline 5.77 & 37.0 & 0.13 & 100.0 & 0.00 & 0.0000 \\
\hline 6.31 & 33.8 & 0.17 & 100.0 & 0.00 & 0.0000 \\
\hline 6.90 & 30.9 & 0.17 & 100.0 & 0.00 & 0.0000 \\
\hline 7.55 & 28.3 & 0.19 & 100.0 & 0.00 & 0.0000 \\
\hline 8.26 & 25.8 & 0.19 & 100.0 & 0.00 & 0.0000 \\
\hline 9.04 & 23.6 & 0.19 & 100.0 & 0.00 & 0.0000 \\
\hline 9.89 & 21.6 & 0.21 & 100.0 & 0.00 & 0.0000 \\
\hline 10.8 & 19.8 & 0.21 & 100.0 & 0.00 & 0.0000 \\
\hline 11.8 & 18.1 & 0.23 & 100.0 & 0.00 & 0.0000 \\
\hline 12.9 & 16.5 & 0.26 & 100.0 & 0.00 & 0.0000 \\
\hline 14.2 & 15.0 & 0.26 & 100.0 & 0.00 & 0.0000 \\
\hline 15.5 & 13.8 & 0.28 & 100.0 & 0.00 & 0.0000 \\
\hline 16.9 & 12.6 & 0.28 & 100.0 & 0.00 & 0.0000 \\
\hline 18.5 & 11.5 & 0.30 & 100.0 & 0.00 & 0.0000 \\
\hline 20.3 & 10.5 & 0.32 & 100.0 & 0.00 & 0.0000 \\
\hline 22.2 & 9.61 & 0.32 & 100.0 & 0.00 & 0.0000 \\
\hline 24.3 & 8.78 & 0.34 & 100.0 & 0.00 & 0.0000 \\
\hline 26.6 & 8.02 & 0.36 & 100.0 & 0.00 & 0.0000 \\
\hline 29.0 & 7.36 & 0.43 & 100.0 & 0.00 & 0.0000 \\
\hline 31.8 & 6.71 & 0.45 & 100.0 & 0.00 & 0.0000 \\
\hline 34.8 & 6.13 & 0.47 & 100.0 & 0.00 & 0.0000 \\
\hline 38.0 & 5.61 & 0.47 & 100.0 & 0.00 & 0.0000 \\
\hline
\end{tabular}


Table 6 (cont.). MICP data for sample 07JRM005 - 9.1a.

\section{Mercury Injection Capillary Pressure 07JRM005 - 9.1a}

\begin{tabular}{|c|c|c|c|c|c|}
\hline $\begin{array}{l}\text { Capillary } \\
\text { Pressure } \\
\text { (psia) } \\
\end{array}$ & $\begin{array}{c}\text { Pore Aperture } \\
\text { Diameter } \\
\text { (microns) } \\
\end{array}$ & $\begin{array}{c}\text { Cumulative } \\
\text { Bulk Vol. (\%) } \\
\end{array}$ & $\begin{array}{l}\text { Wetting Phase } \\
\text { Saturation (\%) }\end{array}$ & $\begin{array}{c}\text { Incremental } \\
\text { Wetting Phase } \\
\text { Change (\%) } \\
\end{array}$ & $\begin{array}{c}\text { Saturation } \\
\text { Change } \\
\text { per psia } \\
\end{array}$ \\
\hline 41.6 & 5.13 & 0.49 & 100.0 & 0.00 & 0.0000 \\
\hline 45.5 & 4.69 & 0.51 & 100.0 & 0.00 & 0.0000 \\
\hline 49.8 & 4.28 & 0.53 & 100.0 & 0.00 & 0.0000 \\
\hline 54.5 & 3.91 & 0.53 & 100.0 & 0.00 & 0.0000 \\
\hline 59.6 & 3.58 & 0.53 & 100.0 & 0.00 & 0.0000 \\
\hline 65.2 & 3.27 & 0.55 & 100.0 & 0.00 & 0.0000 \\
\hline 71.3 & 2.99 & 0.55 & 100.0 & 0.00 & 0.0000 \\
\hline 78.0 & 2.73 & 0.58 & 100.0 & 0.00 & 0.0000 \\
\hline 85.3 & 2.50 & 0.58 & 100.0 & 0.00 & 0.0000 \\
\hline 93.4 & 2.28 & 0.60 & 100.0 & 0.00 & 0.0000 \\
\hline 102 & 2.09 & 0.60 & 100.0 & 0.00 & 0.0000 \\
\hline 112 & 1.90 & 0.62 & 100.0 & 0.00 & 0.0000 \\
\hline 122 & 1.75 & 0.62 & 100.0 & 0.00 & 0.0000 \\
\hline 134 & 1.59 & 0.64 & 100.0 & 0.00 & 0.0000 \\
\hline 146 & 1.46 & 0.64 & 100.0 & 0.00 & 0.0000 \\
\hline 160 & 1.33 & 0.66 & 100.0 & 0.00 & 0.0000 \\
\hline 175 & 1.22 & 0.66 & 100.0 & 0.00 & 0.0000 \\
\hline 191 & 1.12 & 0.68 & 100.0 & 0.00 & 0.0000 \\
\hline 209 & 1.02 & 0.70 & 100.0 & 0.00 & 0.0000 \\
\hline 229 & 0.932 & 0.72 & 100.0 & 0.00 & 0.0000 \\
\hline 251 & 0.850 & 0.75 & 100.0 & 0.00 & 0.0000 \\
\hline 274 & 0.779 & 0.75 & 100.0 & 0.00 & 0.0000 \\
\hline 300 & 0.711 & 0.79 & 100.0 & 0.00 & 0.0000 \\
\hline 328 & 0.650 & 0.81 & 100.0 & 0.00 & 0.0000 \\
\hline 359 & 0.594 & 0.83 & 100.0 & 0.00 & 0.0000 \\
\hline 393 & 0.543 & 0.85 & 100.0 & 0.00 & 0.0000 \\
\hline 430 & 0.496 & 0.90 & 100.0 & 0.00 & 0.0000 \\
\hline 470 & 0.454 & 0.94 & 100.0 & 0.00 & 0.0000 \\
\hline 514 & 0.415 & 0.98 & 100.0 & 0.00 & 0.0000 \\
\hline 563 & 0.379 & 1.02 & 100.0 & 0.00 & 0.0000 \\
\hline 615 & 0.347 & 1.09 & 100.0 & 0.00 & 0.0000 \\
\hline 673 & 0.317 & 1.17 & 100.0 & 0.00 & 0.0000 \\
\hline 736 & 0.290 & 1.26 & 99.6 & 0.41 & 0.0065 \\
\hline 806 & 0.265 & 1.41 & 98.9 & 0.71 & 0.0102 \\
\hline 881 & 0.242 & 1.68 & 97.6 & 1.33 & 0.0177 \\
\hline 964 & 0.221 & 2.15 & 95.3 & 2.24 & 0.0270 \\
\hline 1050 & 0.203 & 3.01 & 91.2 & 4.08 & 0.0475 \\
\hline 1150 & 0.186 & 4.65 & 83.4 & 7.86 & 0.0786 \\
\hline 1260 & 0.169 & 6.42 & 74.9 & 8.47 & 0.0770 \\
\hline 1380 & 0.155 & 8.23 & 66.2 & 8.67 & 0.0723 \\
\hline 1510 & 0.141 & 9.95 & 58.0 & 8.27 & 0.0636 \\
\hline 1650 & 0.129 & 11.17 & 52.1 & 5.82 & 0.0415 \\
\hline
\end{tabular}


Table 6 (cont.). MICP data for sample 07JRM005 - 9.1a.

\section{Mercury Injection Capillary Pressure 07JRM005 - 9.1a}

\begin{tabular}{|c|c|c|c|c|c|}
\hline $\begin{array}{c}\text { Capillary } \\
\text { Pressure } \\
\text { (psia) } \\
\end{array}$ & $\begin{array}{c}\text { Pore Aperture } \\
\text { Diameter } \\
\text { (microns) } \\
\end{array}$ & $\begin{array}{c}\text { Cumulative } \\
\text { Bulk Vol. (\%) } \\
\end{array}$ & $\begin{array}{l}\text { Wetting Phase } \\
\text { Saturation (\%) }\end{array}$ & $\begin{array}{c}\text { Incremental } \\
\text { Wetting Phase } \\
\text { Change (\%) } \\
\end{array}$ & $\begin{array}{c}\text { Saturation } \\
\text { Change } \\
\text { per psia } \\
\end{array}$ \\
\hline 1810 & 0.1179 & 12.15 & 47.4 & 4.69 & 0.0293 \\
\hline 1980 & 0.1077 & 12.98 & 43.5 & 3.98 & 0.0234 \\
\hline 2160 & 0.0988 & 13.68 & 40.1 & 3.37 & 0.0187 \\
\hline 2370 & 0.0900 & 14.37 & 36.8 & 3.27 & 0.0155 \\
\hline 2590 & 0.0824 & 14.92 & 34.2 & 2.65 & 0.0121 \\
\hline 2830 & 0.0754 & 15.43 & 31.7 & 2.45 & 0.0102 \\
\hline 3100 & 0.0688 & 15.90 & 29.5 & 2.24 & 0.0083 \\
\hline 3390 & 0.0629 & 16.33 & 27.4 & 2.04 & 0.0070 \\
\hline 3710 & 0.0575 & 16.73 & 25.5 & 1.94 & 0.0061 \\
\hline 4060 & 0.0525 & 17.09 & 23.8 & 1.73 & 0.0050 \\
\hline 4440 & 0.0480 & 17.44 & 22.1 & 1.63 & 0.0043 \\
\hline 4850 & 0.0440 & 17.71 & 20.8 & 1.33 & 0.0032 \\
\hline 5310 & 0.0402 & 18.01 & 19.4 & 1.43 & 0.0031 \\
\hline 5810 & 0.0367 & 18.29 & 18.1 & 1.33 & 0.0027 \\
\hline 6360 & 0.0335 & 18.54 & 16.8 & 1.22 & 0.0022 \\
\hline 6950 & 0.0307 & 18.76 & 15.8 & 1.02 & 0.0017 \\
\hline 7610 & 0.0280 & 18.99 & 14.7 & 1.12 & 0.0017 \\
\hline 8320 & 0.0256 & 19.20 & 13.7 & 1.02 & 0.0014 \\
\hline 9100 & 0.0234 & 19.44 & 12.6 & 1.12 & 0.0014 \\
\hline 9960 & 0.0214 & 19.63 & 11.6 & 0.92 & 0.0011 \\
\hline 10900 & 0.0196 & 19.80 & 10.8 & 0.82 & 0.0009 \\
\hline 11900 & 0.0179 & 19.99 & 9.9 & 0.92 & 0.0009 \\
\hline 13000 & 0.0164 & 20.16 & 9.1 & 0.82 & 0.0007 \\
\hline 14300 & 0.0149 & 20.33 & 8.3 & 0.82 & 0.0006 \\
\hline 15600 & 0.0137 & 20.48 & 7.6 & 0.71 & 0.0005 \\
\hline 17100 & 0.0125 & 20.63 & 6.8 & 0.71 & 0.0005 \\
\hline 18700 & 0.0114 & 20.78 & 6.1 & 0.71 & 0.0004 \\
\hline 20400 & 0.0105 & 20.91 & 5.5 & 0.61 & 0.0004 \\
\hline 22300 & 0.0096 & 21.04 & 4.9 & 0.61 & 0.0003 \\
\hline 24400 & 0.0087 & 21.17 & 4.3 & 0.61 & 0.0003 \\
\hline 26700 & 0.0080 & 21.27 & 3.8 & 0.51 & 0.0002 \\
\hline 29300 & 0.0073 & 21.40 & 3.2 & 0.61 & 0.0002 \\
\hline 32000 & 0.0067 & 21.49 & 2.8 & 0.41 & 0.0002 \\
\hline 35000 & 0.0061 & 21.57 & 2.3 & 0.41 & 0.0001 \\
\hline 38300 & 0.0056 & 21.68 & 1.8 & 0.51 & 0.0002 \\
\hline 41900 & 0.0051 & 21.76 & 1.4 & 0.41 & 0.0001 \\
\hline 45800 & 0.0047 & 21.83 & 1.1 & 0.31 & 0.0001 \\
\hline 50100 & 0.0043 & 21.91 & 0.7 & 0.41 & 0.0001 \\
\hline 54800 & 0.0039 & 21.98 & 0.4 & 0.31 & 0.0001 \\
\hline 59500 & 0.0036 & 22.06 & 0.0 & 0.41 & 0.0001 \\
\hline
\end{tabular}


Table 7. MICP data for sample 07JRM005 - 16.5.

\section{Mercury Injection Capillary Pressure 07JRM005 - 16.5}

\begin{tabular}{|c|c|c|c|c|c|}
\hline \multicolumn{6}{|c|}{ Sample Information } \\
\hline \multicolumn{3}{|c|}{$\begin{array}{l}\text { Bulk Volume }=5.668 \mathrm{cc} \\
\text { Pore Volume }=\quad 1.2286 \mathrm{cc} \\
\text { Closure }=0.59 \% \mathrm{BV} @ 175 \text { psia }\end{array}$} & \multicolumn{3}{|c|}{$\begin{array}{ll}\text { Porosity }= & 22.1 \% \text { (mercury) } \\
\text { Permeability }= & 0.494 \text { md (mercury) } \\
\text { Median Pore Aperture }=0.3213 \text { microns (diameter) }\end{array}$} \\
\hline $\begin{array}{l}\text { Capillary } \\
\text { Pressure } \\
\text { (psia) }\end{array}$ & $\begin{array}{l}\text { Pore Aperture } \\
\text { Diameter } \\
\text { (microns) }\end{array}$ & $\begin{array}{c}\text { Cumulative } \\
\text { Bulk Vol. (\%) }\end{array}$ & $\begin{array}{l}\text { Wetting Phase } \\
\text { Saturation (\%) }\end{array}$ & $\begin{array}{l}\text { Incremental } \\
\text { Wetting Phase } \\
\text { Change (\%) }\end{array}$ & $\begin{array}{l}\text { Saturation } \\
\text { Change } \\
\text { per psia }\end{array}$ \\
\hline 1.64 & 130 & 0.02 & 100.0 & 0.00 & 0.0000 \\
\hline 1.80 & 119 & 0.02 & 100.0 & 0.00 & 0.0000 \\
\hline 1.96 & 109 & 0.02 & 100.0 & 0.00 & 0.0000 \\
\hline 2.15 & 99.2 & 0.04 & 100.0 & 0.00 & 0.0000 \\
\hline 2.35 & 90.8 & 0.04 & 100.0 & 0.00 & 0.0000 \\
\hline 2.57 & 83.0 & 0.06 & 100.0 & 0.00 & 0.0000 \\
\hline 2.81 & 75.9 & 0.06 & 100.0 & 0.00 & 0.0000 \\
\hline 3.08 & 69.3 & 0.06 & 100.0 & 0.00 & 0.0000 \\
\hline 3.37 & 63.3 & 0.08 & 100.0 & 0.00 & 0.0000 \\
\hline 3.68 & 58.0 & 0.08 & 100.0 & 0.00 & 0.0000 \\
\hline 4.03 & 52.9 & 0.08 & 100.0 & 0.00 & 0.0000 \\
\hline 4.41 & 48.4 & 0.11 & 100.0 & 0.00 & 0.0000 \\
\hline 4.82 & 44.3 & 0.11 & 100.0 & 0.00 & 0.0000 \\
\hline 5.27 & 40.5 & 0.11 & 100.0 & 0.00 & 0.0000 \\
\hline 5.77 & 37.0 & 0.11 & 100.0 & 0.00 & 0.0000 \\
\hline 6.31 & 33.8 & 0.13 & 100.0 & 0.00 & 0.0000 \\
\hline 6.90 & 30.9 & 0.13 & 100.0 & 0.00 & 0.0000 \\
\hline 7.55 & 28.3 & 0.13 & 100.0 & 0.00 & 0.0000 \\
\hline 8.26 & 25.8 & 0.15 & 100.0 & 0.00 & 0.0000 \\
\hline 9.04 & 23.6 & 0.15 & 100.0 & 0.00 & 0.0000 \\
\hline 9.89 & 21.6 & 0.15 & 100.0 & 0.00 & 0.0000 \\
\hline 10.8 & 19.8 & 0.15 & 100.0 & 0.00 & 0.0000 \\
\hline 11.8 & 18.1 & 0.17 & 100.0 & 0.00 & 0.0000 \\
\hline 12.9 & 16.5 & 0.17 & 100.0 & 0.00 & 0.0000 \\
\hline 14.2 & 15.0 & 0.19 & 100.0 & 0.00 & 0.0000 \\
\hline 15.5 & 13.8 & 0.19 & 100.0 & 0.00 & 0.0000 \\
\hline 16.9 & 12.6 & 0.19 & 100.0 & 0.00 & 0.0000 \\
\hline 18.5 & 11.5 & 0.19 & 100.0 & 0.00 & 0.0000 \\
\hline 20.3 & 10.5 & 0.21 & 100.0 & 0.00 & 0.0000 \\
\hline 22.2 & 9.61 & 0.21 & 100.0 & 0.00 & 0.0000 \\
\hline 24.3 & 8.78 & 0.23 & 100.0 & 0.00 & 0.0000 \\
\hline 26.6 & 8.02 & 0.23 & 100.0 & 0.00 & 0.0000 \\
\hline 29.0 & 7.36 & 0.23 & 100.0 & 0.00 & 0.0000 \\
\hline 31.8 & 6.71 & 0.25 & 100.0 & 0.00 & 0.0000 \\
\hline 34.8 & 6.13 & 0.25 & 100.0 & 0.00 & 0.0000 \\
\hline 38.0 & 5.61 & 0.27 & 100.0 & 0.00 & 0.0000 \\
\hline
\end{tabular}


Table 7 (cont.). MICP data for sample 07JRM005 - 16.5.

\section{Mercury Injection Capillary Pressure 07JRM005 - 16.5}

\begin{tabular}{|c|c|c|c|c|c|}
\hline $\begin{array}{l}\text { Capillary } \\
\text { Pressure } \\
\text { (psia) } \\
\end{array}$ & $\begin{array}{l}\text { Pore Aperture } \\
\text { Diameter } \\
\text { (microns) } \\
\end{array}$ & $\begin{array}{c}\text { Cumulative } \\
\text { Bulk Vol. (\%) } \\
\end{array}$ & $\begin{array}{l}\text { Wetting Phase } \\
\text { Saturation (\%) }\end{array}$ & $\begin{array}{l}\text { Incremental } \\
\text { Wetting Phase } \\
\text { Change (\%) } \\
\end{array}$ & $\begin{array}{c}\text { Saturation } \\
\text { Change } \\
\text { per psia }\end{array}$ \\
\hline 41.6 & 5.13 & 0.30 & 100.0 & 0.00 & 0.0000 \\
\hline 45.5 & 4.69 & 0.32 & 100.0 & 0.00 & 0.0000 \\
\hline 49.8 & 4.28 & 0.32 & 100.0 & 0.00 & 0.0000 \\
\hline 54.5 & 3.91 & 0.32 & 100.0 & 0.00 & 0.0000 \\
\hline 59.6 & 3.58 & 0.32 & 100.0 & 0.00 & 0.0000 \\
\hline 65.2 & 3.27 & 0.34 & 100.0 & 0.00 & 0.0000 \\
\hline 71.3 & 2.99 & 0.34 & 100.0 & 0.00 & 0.0000 \\
\hline 78.0 & 2.73 & 0.36 & 100.0 & 0.00 & 0.0000 \\
\hline 85.3 & 2.50 & 0.36 & 100.0 & 0.00 & 0.0000 \\
\hline 93.4 & 2.28 & 0.38 & 100.0 & 0.00 & 0.0000 \\
\hline 102 & 2.09 & 0.38 & 100.0 & 0.00 & 0.0000 \\
\hline 112 & 1.90 & 0.40 & 100.0 & 0.00 & 0.0000 \\
\hline 122 & 1.75 & 0.42 & 100.0 & 0.00 & 0.0000 \\
\hline 134 & 1.59 & 0.44 & 100.0 & 0.00 & 0.0000 \\
\hline 146 & 1.46 & 0.49 & 100.0 & 0.00 & 0.0000 \\
\hline 160 & 1.33 & 0.53 & 100.0 & 0.00 & 0.0000 \\
\hline 175 & 1.22 & 0.59 & 100.0 & 0.00 & 0.0000 \\
\hline 191 & 1.12 & 0.70 & 99.5 & 0.48 & 0.0299 \\
\hline 209 & 1.02 & 0.89 & 98.7 & 0.86 & 0.0478 \\
\hline 229 & 0.932 & 1.18 & 97.3 & 1.34 & 0.0669 \\
\hline 251 & 0.850 & 1.56 & 95.6 & 1.72 & 0.0782 \\
\hline 274 & 0.779 & 2.09 & 93.2 & 2.39 & 0.1039 \\
\hline 300 & 0.711 & 2.91 & 89.5 & 3.73 & 0.1434 \\
\hline 328 & 0.650 & 4.20 & 83.7 & 5.83 & 0.2083 \\
\hline 359 & 0.594 & 5.68 & 77.0 & 6.69 & 0.2159 \\
\hline 393 & 0.543 & 6.94 & 71.2 & 5.74 & 0.1687 \\
\hline 430 & 0.496 & 8.04 & 66.3 & 4.97 & 0.1344 \\
\hline 470 & 0.454 & 8.97 & 62.0 & 4.21 & 0.1052 \\
\hline 514 & 0.415 & 9.77 & 58.4 & 3.63 & 0.0826 \\
\hline 563 & 0.379 & 10.44 & 55.4 & 3.06 & 0.0624 \\
\hline 615 & 0.347 & 11.10 & 52.4 & 2.96 & 0.0570 \\
\hline 673 & 0.317 & 11.71 & 49.6 & 2.77 & 0.0478 \\
\hline 736 & 0.290 & 12.28 & 47.0 & 2.58 & 0.0410 \\
\hline 806 & 0.265 & 12.81 & 44.6 & 2.39 & 0.0341 \\
\hline 881 & 0.242 & 13.34 & 42.3 & 2.39 & 0.0319 \\
\hline 964 & 0.221 & 13.88 & 39.8 & 2.49 & 0.0299 \\
\hline 1050 & 0.203 & 14.41 & 37.4 & 2.39 & 0.0278 \\
\hline 1150 & 0.186 & 14.81 & 35.6 & 1.82 & 0.0182 \\
\hline 1260 & 0.169 & 15.17 & 33.9 & 1.63 & 0.0148 \\
\hline 1380 & 0.155 & 15.55 & 32.2 & 1.72 & 0.0143 \\
\hline 1510 & 0.141 & 15.91 & 30.6 & 1.63 & 0.0125 \\
\hline 1650 & 0.129 & 16.25 & 29.1 & 1.53 & 0.0109 \\
\hline
\end{tabular}


Table 7 (cont.). MICP data for sample 07JRM005 - 16.5.

\section{Mercury Injection Capillary Pressure 07JRM005 - 16.5}

\begin{tabular}{|c|c|c|c|c|c|}
\hline $\begin{array}{l}\text { Capillary } \\
\text { Pressure } \\
\text { (psia) } \\
\end{array}$ & $\begin{array}{l}\text { Pore Aperture } \\
\text { Diameter } \\
\text { (microns) } \\
\end{array}$ & $\begin{array}{c}\text { Cumulative } \\
\text { Bulk Vol. (\%) } \\
\end{array}$ & $\begin{array}{l}\text { Wetting Phase } \\
\text { Saturation (\%) }\end{array}$ & $\begin{array}{c}\text { Incremental } \\
\text { Wetting Phase } \\
\text { Change (\%) } \\
\end{array}$ & $\begin{array}{c}\text { Saturation } \\
\text { Change } \\
\text { per psia } \\
\end{array}$ \\
\hline 1810 & 0.1179 & 16.58 & 27.5 & 1.53 & 0.0096 \\
\hline 1980 & 0.1077 & 16.90 & 26.1 & 1.43 & 0.0084 \\
\hline 2160 & 0.0988 & 17.20 & 24.8 & 1.34 & 0.0074 \\
\hline 2370 & 0.0900 & 17.49 & 23.4 & 1.34 & 0.0064 \\
\hline 2590 & 0.0824 & 17.77 & 22.2 & 1.24 & 0.0056 \\
\hline 2830 & 0.0754 & 18.04 & 20.9 & 1.24 & 0.0052 \\
\hline 3100 & 0.0688 & 18.29 & 19.8 & 1.15 & 0.0042 \\
\hline 3390 & 0.0629 & 18.53 & 18.7 & 1.05 & 0.0036 \\
\hline 3710 & 0.0575 & 18.76 & 17.7 & 1.05 & 0.0033 \\
\hline 4060 & 0.0525 & 18.97 & 16.7 & 0.96 & 0.0027 \\
\hline 4440 & 0.0480 & 19.18 & 15.8 & 0.96 & 0.0025 \\
\hline 4850 & 0.0440 & 19.35 & 15.0 & 0.76 & 0.0019 \\
\hline 5310 & 0.0402 & 19.54 & 14.1 & 0.86 & 0.0019 \\
\hline 5810 & 0.0367 & 19.73 & 13.3 & 0.86 & 0.0017 \\
\hline 6360 & 0.0335 & 19.90 & 12.5 & 0.76 & 0.0014 \\
\hline 6950 & 0.0307 & 20.07 & 11.8 & 0.76 & 0.0013 \\
\hline 7610 & 0.0280 & 20.23 & 11.0 & 0.76 & 0.0012 \\
\hline 8320 & 0.0256 & 20.38 & 10.3 & 0.67 & 0.0009 \\
\hline 9100 & 0.0234 & 20.55 & 9.6 & 0.76 & 0.0010 \\
\hline 9960 & 0.0214 & 20.70 & 8.9 & 0.67 & 0.0008 \\
\hline 10900 & 0.0196 & 20.85 & 8.2 & 0.67 & 0.0007 \\
\hline 11900 & 0.0179 & 20.97 & 7.6 & 0.57 & 0.0006 \\
\hline 13000 & 0.0164 & 21.10 & 7.1 & 0.57 & 0.0005 \\
\hline 14300 & 0.0149 & 21.25 & 6.4 & 0.67 & 0.0005 \\
\hline 15600 & 0.0137 & 21.37 & 5.8 & 0.57 & 0.0004 \\
\hline 17100 & 0.0125 & 21.48 & 5.4 & 0.48 & 0.0003 \\
\hline 18700 & 0.0114 & 21.61 & 4.8 & 0.57 & 0.0004 \\
\hline 20400 & 0.0105 & 21.71 & 4.3 & 0.48 & 0.0003 \\
\hline 22300 & 0.0096 & 21.82 & 3.8 & 0.48 & 0.0003 \\
\hline 24400 & 0.0087 & 21.92 & 3.3 & 0.48 & 0.0002 \\
\hline 26700 & 0.0080 & 22.01 & 3.0 & 0.38 & 0.0002 \\
\hline 29300 & 0.0073 & 22.11 & 2.5 & 0.48 & 0.0002 \\
\hline 32000 & 0.0067 & 22.20 & 2.1 & 0.38 & 0.0001 \\
\hline 35000 & 0.0061 & 22.26 & 1.8 & 0.29 & 0.0001 \\
\hline 38300 & 0.0056 & 22.34 & 1.4 & 0.38 & 0.0001 \\
\hline 41900 & 0.0051 & 22.41 & 1.1 & 0.29 & 0.0001 \\
\hline 45800 & 0.0047 & 22.47 & 0.9 & 0.29 & 0.0001 \\
\hline 50100 & 0.0043 & 22.53 & 0.6 & 0.29 & 0.0001 \\
\hline 54800 & 0.0039 & 22.58 & 0.4 & 0.19 & 0.0000 \\
\hline 59500 & 0.0036 & 22.66 & 0.0 & 0.38 & 0.0001 \\
\hline
\end{tabular}


Table 8. MICP data for sample 07JRM006 - 18.3a.

\section{Mercury Injection Capillary Pressure 07JRM006 - 18.3a}

\begin{tabular}{|c|c|c|c|c|c|}
\hline \multicolumn{3}{|c|}{ Sample Information } & \multirow{2}{*}{\multicolumn{3}{|c|}{$\begin{array}{l}\text { Porosity }=\quad 22.1 \% \text { (mercury) } \\
\text { Permeability }=\quad 0.0682 \text { md (mercury) } \\
\text { Median Pore Aperture }=0.1013 \text { microns (diameter) }\end{array}$}} \\
\hline $\begin{array}{l}\text { Bulk Volume } \\
\text { Pore Volume } \\
\text { Closure }=1.42\end{array}$ & $\begin{array}{c}8.2225 \mathrm{cc} \\
1.8167 \mathrm{cc} \\
\% \mathrm{BV} @ 881 \text { psia }\end{array}$ & & & & \\
\hline $\begin{array}{l}\text { Capillary } \\
\text { Pressure } \\
\text { (psia) } \\
\end{array}$ & $\begin{array}{l}\text { Pore Aperture } \\
\text { Diameter } \\
\text { (microns) } \\
\end{array}$ & $\begin{array}{c}\text { Cumulative } \\
\text { Bulk Vol. (\%) } \\
\end{array}$ & $\begin{array}{l}\text { Wetting Phase } \\
\text { Saturation (\%) }\end{array}$ & $\begin{array}{l}\text { Incremental } \\
\text { Wetting Phase } \\
\text { Change (\%) } \\
\end{array}$ & $\begin{array}{l}\text { Saturation } \\
\text { Change } \\
\text { per psia } \\
\end{array}$ \\
\hline 1.64 & 130 & 0.00 & 100.0 & 0.00 & 0.0000 \\
\hline 1.80 & 119 & 0.02 & 100.0 & 0.00 & 0.0000 \\
\hline 1.96 & 109 & 0.02 & 100.0 & 0.00 & 0.0000 \\
\hline 2.15 & 99.2 & 0.02 & 100.0 & 0.00 & 0.0000 \\
\hline 2.35 & 90.8 & 0.04 & 100.0 & 0.00 & 0.0000 \\
\hline 2.57 & 83.0 & 0.04 & 100.0 & 0.00 & 0.0000 \\
\hline 2.81 & 75.9 & 0.04 & 100.0 & 0.00 & 0.0000 \\
\hline 3.08 & 69.3 & 0.04 & 100.0 & 0.00 & 0.0000 \\
\hline 3.37 & 63.3 & 0.04 & 100.0 & 0.00 & 0.0000 \\
\hline 3.68 & 58.0 & 0.04 & 100.0 & 0.00 & 0.0000 \\
\hline 4.03 & 52.9 & 0.04 & 100.0 & 0.00 & 0.0000 \\
\hline 4.41 & 48.4 & 0.04 & 100.0 & 0.00 & 0.0000 \\
\hline 4.82 & 44.3 & 0.04 & 100.0 & 0.00 & 0.0000 \\
\hline 5.27 & 40.5 & 0.04 & 100.0 & 0.00 & 0.0000 \\
\hline 5.77 & 37.0 & 0.04 & 100.0 & 0.00 & 0.0000 \\
\hline 6.31 & 33.8 & 0.07 & 100.0 & 0.00 & 0.0000 \\
\hline 6.90 & 30.9 & 0.13 & 100.0 & 0.00 & 0.0000 \\
\hline 7.55 & 28.3 & 0.13 & 100.0 & 0.00 & 0.0000 \\
\hline 8.26 & 25.8 & 0.15 & 100.0 & 0.00 & 0.0000 \\
\hline 9.04 & 23.6 & 0.15 & 100.0 & 0.00 & 0.0000 \\
\hline 9.89 & 21.6 & 0.15 & 100.0 & 0.00 & 0.0000 \\
\hline 10.8 & 19.8 & 0.17 & 100.0 & 0.00 & 0.0000 \\
\hline 11.8 & 18.1 & 0.17 & 100.0 & 0.00 & 0.0000 \\
\hline 12.9 & 16.5 & 0.17 & 100.0 & 0.00 & 0.0000 \\
\hline 14.2 & 15.0 & 0.20 & 100.0 & 0.00 & 0.0000 \\
\hline 15.5 & 13.8 & 0.20 & 100.0 & 0.00 & 0.0000 \\
\hline 16.9 & 12.6 & 0.20 & 100.0 & 0.00 & 0.0000 \\
\hline 18.5 & 11.5 & 0.22 & 100.0 & 0.00 & 0.0000 \\
\hline 20.3 & 10.5 & 0.22 & 100.0 & 0.00 & 0.0000 \\
\hline 22.2 & 9.61 & 0.22 & 100.0 & 0.00 & 0.0000 \\
\hline 24.3 & 8.78 & 0.24 & 100.0 & 0.00 & 0.0000 \\
\hline 26.6 & 8.02 & 0.24 & 100.0 & 0.00 & 0.0000 \\
\hline 29.0 & 7.36 & 0.26 & 100.0 & 0.00 & 0.0000 \\
\hline 31.8 & 6.71 & 0.26 & 100.0 & 0.00 & 0.0000 \\
\hline 34.8 & 6.13 & 0.28 & 100.0 & 0.00 & 0.0000 \\
\hline 38.0 & 5.61 & 0.28 & 100.0 & 0.00 & 0.0000 \\
\hline
\end{tabular}


Table 8 (cont.). MICP data for sample 07JRM006 - 18.3a.

\section{Mercury Injection Capillary Pressure 07JRM006 - 18.3a}

\begin{tabular}{|c|c|c|c|c|c|}
\hline $\begin{array}{c}\text { Capillary } \\
\text { Pressure } \\
\text { (psia) } \\
\end{array}$ & $\begin{array}{c}\text { Pore Aperture } \\
\text { Diameter } \\
\text { (microns) } \\
\end{array}$ & $\begin{array}{c}\text { Cumulative } \\
\text { Bulk Vol. (\%) } \\
\end{array}$ & $\begin{array}{l}\text { Wetting Phase } \\
\text { Saturation (\%) }\end{array}$ & $\begin{array}{c}\text { Incremental } \\
\text { Wetting Phase } \\
\text { Change (\%) } \\
\end{array}$ & $\begin{array}{c}\text { Saturation } \\
\text { Change } \\
\text { per psia } \\
\end{array}$ \\
\hline 41.6 & 5.13 & 0.31 & 100.0 & 0.00 & 0.0000 \\
\hline 45.5 & 4.69 & 0.33 & 100.0 & 0.00 & 0.0000 \\
\hline 49.8 & 4.28 & 0.33 & 100.0 & 0.00 & 0.0000 \\
\hline 54.5 & 3.91 & 0.35 & 100.0 & 0.00 & 0.0000 \\
\hline 59.6 & 3.58 & 0.35 & 100.0 & 0.00 & 0.0000 \\
\hline 65.2 & 3.27 & 0.37 & 100.0 & 0.00 & 0.0000 \\
\hline 71.3 & 2.99 & 0.37 & 100.0 & 0.00 & 0.0000 \\
\hline 78.0 & 2.73 & 0.37 & 100.0 & 0.00 & 0.0000 \\
\hline 85.3 & 2.50 & 0.39 & 100.0 & 0.00 & 0.0000 \\
\hline 93.4 & 2.28 & 0.41 & 100.0 & 0.00 & 0.0000 \\
\hline 102 & 2.09 & 0.41 & 100.0 & 0.00 & 0.0000 \\
\hline 112 & 1.90 & 0.46 & 100.0 & 0.00 & 0.0000 \\
\hline 122 & 1.75 & 0.48 & 100.0 & 0.00 & 0.0000 \\
\hline 134 & 1.59 & 0.48 & 100.0 & 0.00 & 0.0000 \\
\hline 146 & 1.46 & 0.50 & 100.0 & 0.00 & 0.0000 \\
\hline 160 & 1.33 & 0.52 & 100.0 & 0.00 & 0.0000 \\
\hline 175 & 1.22 & 0.59 & 100.0 & 0.00 & 0.0000 \\
\hline 191 & 1.12 & 0.61 & 100.0 & 0.00 & 0.0000 \\
\hline 209 & 1.02 & 0.63 & 100.0 & 0.00 & 0.0000 \\
\hline 229 & 0.932 & 0.65 & 100.0 & 0.00 & 0.0000 \\
\hline 251 & 0.850 & 0.68 & 100.0 & 0.00 & 0.0000 \\
\hline 274 & 0.779 & 0.72 & 100.0 & 0.00 & 0.0000 \\
\hline 300 & 0.711 & 0.74 & 100.0 & 0.00 & 0.0000 \\
\hline 328 & 0.650 & 0.76 & 100.0 & 0.00 & 0.0000 \\
\hline 359 & 0.594 & 0.81 & 100.0 & 0.00 & 0.0000 \\
\hline 393 & 0.543 & 0.83 & 100.0 & 0.00 & 0.0000 \\
\hline 430 & 0.496 & 0.87 & 100.0 & 0.00 & 0.0000 \\
\hline 470 & 0.454 & 0.89 & 100.0 & 0.00 & 0.0000 \\
\hline 514 & 0.415 & 0.94 & 100.0 & 0.00 & 0.0000 \\
\hline 563 & 0.379 & 0.98 & 100.0 & 0.00 & 0.0000 \\
\hline 615 & 0.347 & 1.05 & 100.0 & 0.00 & 0.0000 \\
\hline 673 & 0.317 & 1.11 & 100.0 & 0.00 & 0.0000 \\
\hline 736 & 0.290 & 1.20 & 100.0 & 0.00 & 0.0000 \\
\hline 806 & 0.265 & 1.29 & 100.0 & 0.00 & 0.0000 \\
\hline 881 & 0.242 & 1.42 & 100.0 & 0.00 & 0.0000 \\
\hline 964 & 0.221 & 1.57 & 99.3 & 0.69 & 0.0083 \\
\hline 1050 & 0.203 & 1.85 & 98.0 & 1.28 & 0.0149 \\
\hline 1150 & 0.186 & 2.36 & 95.8 & 2.27 & 0.0227 \\
\hline 1260 & 0.169 & 3.21 & 91.9 & 3.85 & 0.0350 \\
\hline 1380 & 0.155 & 4.82 & 84.6 & 7.31 & 0.0609 \\
\hline 1510 & 0.141 & 6.67 & 76.2 & 8.39 & 0.0645 \\
\hline 1650 & 0.129 & 8.64 & 67.3 & 8.88 & 0.0635 \\
\hline
\end{tabular}


Table 8 (cont.). MICP data for sample 07JRM006 - 18.3a.

\section{Mercury Injection Capillary Pressure 07JRM006 - 18.3a}

\begin{tabular}{|c|c|c|c|c|c|}
\hline $\begin{array}{c}\text { Capillary } \\
\text { Pressure } \\
\text { (psia) } \\
\end{array}$ & $\begin{array}{c}\text { Pore Aperture } \\
\text { Diameter } \\
\text { (microns) } \\
\end{array}$ & $\begin{array}{c}\text { Cumulative } \\
\text { Bulk Vol. (\%) } \\
\end{array}$ & $\begin{array}{l}\text { Wetting Phase } \\
\text { Saturation (\%) }\end{array}$ & $\begin{array}{c}\text { Incremental } \\
\text { Wetting Phase } \\
\text { Change (\%) } \\
\end{array}$ & $\begin{array}{c}\text { Saturation } \\
\text { Change } \\
\text { per psia }\end{array}$ \\
\hline 1810 & 0.1179 & 10.32 & 59.7 & 7.60 & 0.0475 \\
\hline 1980 & 0.1077 & 11.67 & 53.6 & 6.12 & 0.0360 \\
\hline 2160 & 0.0988 & 12.78 & 48.6 & 5.03 & 0.0280 \\
\hline 2370 & 0.0900 & 13.85 & 43.7 & 4.84 & 0.0230 \\
\hline 2590 & 0.0824 & 14.72 & 39.8 & 3.95 & 0.0179 \\
\hline 2830 & 0.0754 & 15.49 & 36.3 & 3.46 & 0.0144 \\
\hline 3100 & 0.0688 & 16.21 & 33.1 & 3.26 & 0.0121 \\
\hline 3390 & 0.0629 & 16.82 & 30.3 & 2.76 & 0.0095 \\
\hline 3710 & 0.0575 & 17.36 & 27.8 & 2.47 & 0.0077 \\
\hline 4060 & 0.0525 & 17.84 & 25.7 & 2.17 & 0.0062 \\
\hline 4440 & 0.0480 & 18.30 & 23.6 & 2.07 & 0.0055 \\
\hline 4850 & 0.0440 & 18.67 & 21.9 & 1.68 & 0.0041 \\
\hline 5310 & 0.0402 & 19.04 & 20.2 & 1.68 & 0.0036 \\
\hline 5810 & 0.0367 & 19.37 & 18.8 & 1.48 & 0.0030 \\
\hline 6360 & 0.0335 & 19.67 & 17.4 & 1.38 & 0.0025 \\
\hline 6950 & 0.0307 & 19.98 & 16.0 & 1.38 & 0.0023 \\
\hline 7610 & 0.0280 & 20.24 & 14.8 & 1.18 & 0.0018 \\
\hline 8320 & 0.0256 & 20.48 & 13.7 & 1.09 & 0.0015 \\
\hline 9100 & 0.0234 & 20.72 & 12.6 & 1.09 & 0.0014 \\
\hline 9960 & 0.0214 & 20.94 & 11.6 & 0.99 & 0.0011 \\
\hline 10900 & 0.0196 & 21.13 & 10.8 & 0.89 & 0.0009 \\
\hline 11900 & 0.0179 & 21.33 & 9.9 & 0.89 & 0.0009 \\
\hline 13000 & 0.0164 & 21.51 & 9.1 & 0.79 & 0.0007 \\
\hline 14300 & 0.0149 & 21.68 & 8.3 & 0.79 & 0.0006 \\
\hline 15600 & 0.0137 & 21.83 & 7.6 & 0.69 & 0.0005 \\
\hline 17100 & 0.0125 & 21.99 & 6.9 & 0.69 & 0.0005 \\
\hline 18700 & 0.0114 & 22.14 & 6.2 & 0.69 & 0.0004 \\
\hline 20400 & 0.0105 & 22.27 & 5.6 & 0.59 & 0.0003 \\
\hline 22300 & 0.0096 & 22.40 & 5.0 & 0.59 & 0.0003 \\
\hline 24400 & 0.0087 & 22.53 & 4.4 & 0.59 & 0.0003 \\
\hline 26700 & 0.0080 & 22.66 & 3.8 & 0.59 & 0.0003 \\
\hline 29300 & 0.0073 & 22.77 & 3.4 & 0.49 & 0.0002 \\
\hline 32000 & 0.0067 & 22.88 & 2.9 & 0.49 & 0.0002 \\
\hline 35000 & 0.0061 & 22.97 & 2.5 & 0.39 & 0.0001 \\
\hline 38300 & 0.0056 & 23.08 & 2.0 & 0.49 & 0.0001 \\
\hline 41900 & 0.0051 & 23.16 & 1.6 & 0.39 & 0.0001 \\
\hline 45800 & 0.0047 & 23.25 & 1.2 & 0.39 & 0.0001 \\
\hline 50100 & 0.0043 & 23.32 & 0.9 & 0.30 & 0.0001 \\
\hline 54800 & 0.0039 & 23.42 & 0.4 & 0.49 & 0.0001 \\
\hline 59500 & 0.0036 & 23.51 & 0.0 & 0.39 & 0.0001 \\
\hline
\end{tabular}


Table 9. MICP data for sample 07JRM008 - 26.5a.

\section{Mercury Injection Capillary Pressure 07JRM008 - 26.5a}

\begin{tabular}{|c|c|c|c|c|c|}
\hline \multicolumn{6}{|c|}{ Sample Information } \\
\hline \multirow{3}{*}{\multicolumn{3}{|c|}{$\begin{array}{lr}\text { Bulk Volume }= & 7.0014 \mathrm{cc} \\
\text { Pore Volume }= & 1.6765 \mathrm{cc} \\
\text { Closure }=0.70 \% \mathrm{BV} @ 300 \mathrm{psia} \\
\end{array}$}} & \multirow{3}{*}{\multicolumn{3}{|c|}{$\begin{array}{l}\text { Porosity }=\quad 24.0 \% \text { (mercury) } \\
\text { Permeability }=0.422 \text { md (mercury) } \\
\text { Median Pore Aperture }=0.290 \text { microns (diameter }\end{array}$}} \\
\hline & & & & & \\
\hline & & & & & \\
\hline Capillary & Pore Aperture & & & Incremental & Saturation \\
\hline $\begin{array}{c}\text { Pressure } \\
\text { (psia) }\end{array}$ & $\begin{array}{l}\text { Diameter } \\
\text { (microns) } \\
\end{array}$ & $\begin{array}{c}\text { Cumulative } \\
\text { Bulk Vol. (\%) }\end{array}$ & $\begin{array}{l}\text { Wetting Phase } \\
\text { Saturation (\%) }\end{array}$ & $\begin{array}{c}\text { Wetting Phase } \\
\text { Change (\%) }\end{array}$ & $\begin{array}{l}\text { Change } \\
\text { per psia }\end{array}$ \\
\hline 1.64 & 130 & 0.02 & 100.0 & 0.00 & 0.0000 \\
\hline 1.80 & 119 & 0.02 & 100.0 & 0.00 & 0.0000 \\
\hline 1.96 & 109 & 0.02 & 100.0 & 0.00 & 0.0000 \\
\hline 2.15 & 99.2 & 0.02 & 100.0 & 0.00 & 0.0000 \\
\hline 2.35 & 90.8 & 0.04 & 100.0 & 0.00 & 0.0000 \\
\hline 2.57 & 83.0 & 0.04 & 100.0 & 0.00 & 0.0000 \\
\hline 2.81 & 75.9 & 0.04 & 100.0 & 0.00 & 0.0000 \\
\hline 3.08 & 69.3 & 0.06 & 100.0 & 0.00 & 0.0000 \\
\hline 3.37 & 63.3 & 0.06 & 100.0 & 0.00 & 0.0000 \\
\hline 3.68 & 58.0 & 0.06 & 100.0 & 0.00 & 0.0000 \\
\hline 4.03 & 52.9 & 0.08 & 100.0 & 0.00 & 0.0000 \\
\hline 4.41 & 48.4 & 0.08 & 100.0 & 0.00 & 0.0000 \\
\hline 4.82 & 44.3 & 0.08 & 100.0 & 0.00 & 0.0000 \\
\hline 5.27 & 40.5 & 0.10 & 100.0 & 0.00 & 0.0000 \\
\hline 5.77 & 37.0 & 0.10 & 100.0 & 0.00 & 0.0000 \\
\hline 6.31 & 33.8 & 0.10 & 100.0 & 0.00 & 0.0000 \\
\hline 6.90 & 30.9 & 0.12 & 100.0 & 0.00 & 0.0000 \\
\hline 7.55 & 28.3 & 0.12 & 100.0 & 0.00 & 0.0000 \\
\hline 8.26 & 25.8 & 0.12 & 100.0 & 0.00 & 0.0000 \\
\hline 9.04 & 23.6 & 0.14 & 100.0 & 0.00 & 0.0000 \\
\hline 9.89 & 21.6 & 0.14 & 100.0 & 0.00 & 0.0000 \\
\hline 10.8 & 19.8 & 0.16 & 100.0 & 0.00 & 0.0000 \\
\hline 11.8 & 18.1 & 0.16 & 100.0 & 0.00 & 0.0000 \\
\hline 12.9 & 16.5 & 0.16 & 100.0 & 0.00 & 0.0000 \\
\hline 14.2 & 15.0 & 0.18 & 100.0 & 0.00 & 0.0000 \\
\hline 15.5 & 13.8 & 0.18 & 100.0 & 0.00 & 0.0000 \\
\hline 16.9 & 12.6 & 0.20 & 100.0 & 0.00 & 0.0000 \\
\hline 18.5 & 11.5 & 0.20 & 100.0 & 0.00 & 0.0000 \\
\hline 20.3 & 10.5 & 0.22 & 100.0 & 0.00 & 0.0000 \\
\hline 22.2 & 9.61 & 0.22 & 100.0 & 0.00 & 0.0000 \\
\hline 24.3 & 8.78 & 0.25 & 100.0 & 0.00 & 0.0000 \\
\hline 26.6 & 8.02 & 0.25 & 100.0 & 0.00 & 0.0000 \\
\hline 29.0 & 7.36 & 0.27 & 100.0 & 0.00 & 0.0000 \\
\hline 31.8 & 6.71 & 0.29 & 100.0 & 0.00 & 0.0000 \\
\hline 34.8 & 6.13 & 0.29 & 100.0 & 0.00 & 0.0000 \\
\hline 38.0 & 5.61 & 0.31 & 100.0 & 0.00 & 0.0000 \\
\hline
\end{tabular}


Table 9 (cont.). MICP data for sample 07JRM008 - 26.5a.

\section{Mercury Injection Capillary Pressure 07JRM008 - 26.5a}

\begin{tabular}{|c|c|c|c|c|c|}
\hline $\begin{array}{c}\text { Capillary } \\
\text { Pressure } \\
\text { (psia) } \\
\end{array}$ & $\begin{array}{c}\text { Pore Aperture } \\
\text { Diameter } \\
\text { (microns) } \\
\end{array}$ & $\begin{array}{c}\text { Cumulative } \\
\text { Bulk Vol. (\%) } \\
\end{array}$ & $\begin{array}{l}\text { Wetting Phase } \\
\text { Saturation (\%) }\end{array}$ & $\begin{array}{c}\text { Incremental } \\
\text { Wetting Phase } \\
\text { Change (\%) } \\
\end{array}$ & $\begin{array}{c}\text { Saturation } \\
\text { Change } \\
\text { per psia } \\
\end{array}$ \\
\hline 41.6 & 5.13 & 0.31 & 100.0 & 0.00 & 0.0000 \\
\hline 45.5 & 4.69 & 0.33 & 100.0 & 0.00 & 0.0000 \\
\hline 49.8 & 4.28 & 0.33 & 100.0 & 0.00 & 0.0000 \\
\hline 54.5 & 3.91 & 0.35 & 100.0 & 0.00 & 0.0000 \\
\hline 59.6 & 3.58 & 0.35 & 100.0 & 0.00 & 0.0000 \\
\hline 65.2 & 3.27 & 0.35 & 100.0 & 0.00 & 0.0000 \\
\hline 71.3 & 2.99 & 0.35 & 100.0 & 0.00 & 0.0000 \\
\hline 78.0 & 2.73 & 0.37 & 100.0 & 0.00 & 0.0000 \\
\hline 85.3 & 2.50 & 0.37 & 100.0 & 0.00 & 0.0000 \\
\hline 93.4 & 2.28 & 0.39 & 100.0 & 0.00 & 0.0000 \\
\hline 102 & 2.09 & 0.39 & 100.0 & 0.00 & 0.0000 \\
\hline 112 & 1.90 & 0.41 & 100.0 & 0.00 & 0.0000 \\
\hline 122 & 1.75 & 0.43 & 100.0 & 0.00 & 0.0000 \\
\hline 134 & 1.59 & 0.43 & 100.0 & 0.00 & 0.0000 \\
\hline 146 & 1.46 & 0.45 & 100.0 & 0.00 & 0.0000 \\
\hline 160 & 1.33 & 0.47 & 100.0 & 0.00 & 0.0000 \\
\hline 175 & 1.22 & 0.49 & 100.0 & 0.00 & 0.0000 \\
\hline 191 & 1.12 & 0.51 & 100.0 & 0.00 & 0.0000 \\
\hline 209 & 1.02 & 0.53 & 100.0 & 0.00 & 0.0000 \\
\hline 229 & 0.932 & 0.55 & 100.0 & 0.00 & 0.0000 \\
\hline 251 & 0.850 & 0.59 & 100.0 & 0.00 & 0.0000 \\
\hline 274 & 0.779 & 0.63 & 100.0 & 0.00 & 0.0000 \\
\hline 300 & 0.711 & 0.70 & 100.0 & 0.00 & 0.0000 \\
\hline 328 & 0.650 & 0.80 & 99.6 & 0.43 & 0.0152 \\
\hline 359 & 0.594 & 0.98 & 98.8 & 0.77 & 0.0248 \\
\hline 393 & 0.543 & 1.55 & 96.4 & 2.39 & 0.0703 \\
\hline 430 & 0.496 & 3.46 & 88.5 & 7.94 & 0.2146 \\
\hline 470 & 0.454 & 6.05 & 77.6 & 10.85 & 0.2711 \\
\hline 514 & 0.415 & 7.89 & 69.9 & 7.69 & 0.1747 \\
\hline 563 & 0.379 & 9.39 & 63.7 & 6.23 & 0.1272 \\
\hline 615 & 0.347 & 10.61 & 58.6 & 5.12 & 0.0985 \\
\hline 673 & 0.317 & 11.70 & 54.1 & 4.53 & 0.0780 \\
\hline 736 & 0.290 & 12.68 & 50.0 & 4.10 & 0.0651 \\
\hline 806 & 0.265 & 13.56 & 46.3 & 3.67 & 0.0525 \\
\hline 881 & 0.242 & 14.38 & 42.9 & 3.42 & 0.0455 \\
\hline 964 & 0.221 & 15.15 & 39.6 & 3.25 & 0.0391 \\
\hline 1050 & 0.203 & 15.83 & 36.8 & 2.82 & 0.0328 \\
\hline 1150 & 0.186 & 16.52 & 33.9 & 2.90 & 0.0290 \\
\hline 1260 & 0.169 & 17.10 & 31.5 & 2.39 & 0.0217 \\
\hline 1380 & 0.155 & 17.59 & 29.5 & 2.05 & 0.0171 \\
\hline 1510 & 0.141 & 18.08 & 27.4 & 2.05 & 0.0158 \\
\hline 1650 & 0.129 & 18.51 & 25.6 & 1.79 & 0.0128 \\
\hline
\end{tabular}


Table 9 (cont.). MICP data for sample 07JRM008 - $26.5 a$.

\section{Mercury Injection Capillary Pressure 07JRM008 - 26.5a}

\begin{tabular}{|c|c|c|c|c|c|}
\hline $\begin{array}{c}\text { Capillary } \\
\text { Pressure } \\
\text { (psia) } \\
\end{array}$ & $\begin{array}{c}\text { Pore Aperture } \\
\text { Diameter } \\
\text { (microns) }\end{array}$ & $\begin{array}{c}\text { Cumulative } \\
\text { Bulk Vol. (\%) } \\
\end{array}$ & $\begin{array}{l}\text { Wetting Phase } \\
\text { Saturation (\%) }\end{array}$ & $\begin{array}{c}\text { Incremental } \\
\text { Wetting Phase } \\
\text { Change (\%) }\end{array}$ & $\begin{array}{c}\text { Saturation } \\
\text { Change } \\
\text { per psia }\end{array}$ \\
\hline 1810 & 0.1179 & 18.94 & 23.8 & 1.79 & 0.0112 \\
\hline 1980 & 0.1077 & 19.30 & 22.3 & 1.54 & 0.0090 \\
\hline 2160 & 0.0988 & 19.65 & 20.8 & 1.45 & 0.0081 \\
\hline 2370 & 0.0900 & 20.00 & 19.4 & 1.45 & 0.0069 \\
\hline 2590 & 0.0824 & 20.29 & 18.2 & 1.20 & 0.0054 \\
\hline 2830 & 0.0754 & 20.57 & 17.0 & 1.20 & 0.0050 \\
\hline 3100 & 0.0688 & 20.84 & 15.9 & 1.11 & 0.0041 \\
\hline 3390 & 0.0629 & 21.08 & 14.9 & 1.02 & 0.0035 \\
\hline 3710 & 0.0575 & 21.33 & 13.8 & 1.02 & 0.0032 \\
\hline 4060 & 0.0525 & 21.53 & 13.0 & 0.85 & 0.0024 \\
\hline 4440 & 0.0480 & 21.74 & 12.1 & 0.85 & 0.0022 \\
\hline 4850 & 0.0440 & 21.92 & 11.4 & 0.77 & 0.0019 \\
\hline 5310 & 0.0402 & 22.08 & 10.7 & 0.68 & 0.0015 \\
\hline 5810 & 0.0367 & 22.27 & 9.9 & 0.77 & 0.0015 \\
\hline 6360 & 0.0335 & 22.43 & 9.2 & 0.68 & 0.0012 \\
\hline 6950 & 0.0307 & 22.58 & 8.6 & 0.60 & 0.0010 \\
\hline 7610 & 0.0280 & 22.72 & 8.0 & 0.60 & 0.0009 \\
\hline 8320 & 0.0256 & 22.86 & 7.4 & 0.60 & 0.0008 \\
\hline 9100 & 0.0234 & 22.98 & 6.9 & 0.51 & 0.0007 \\
\hline 9960 & 0.0214 & 23.11 & 6.4 & 0.51 & 0.0006 \\
\hline 10900 & 0.0196 & 23.23 & 5.9 & 0.51 & 0.0005 \\
\hline 11900 & 0.0179 & 23.33 & 5.5 & 0.43 & 0.0004 \\
\hline 13000 & 0.0164 & 23.43 & 5.0 & 0.43 & 0.0004 \\
\hline 14300 & 0.0149 & 23.56 & 4.5 & 0.51 & 0.0004 \\
\hline 15600 & 0.0137 & 23.64 & 4.2 & 0.34 & 0.0003 \\
\hline 17100 & 0.0125 & 23.74 & 3.8 & 0.43 & 0.0003 \\
\hline 18700 & 0.0114 & 23.84 & 3.3 & 0.43 & 0.0003 \\
\hline 20400 & 0.0105 & 23.93 & 3.0 & 0.34 & 0.0002 \\
\hline 22300 & 0.0096 & 24.01 & 2.6 & 0.34 & 0.0002 \\
\hline 24400 & 0.0087 & 24.07 & 2.4 & 0.26 & 0.0001 \\
\hline 26700 & 0.0080 & 24.15 & 2.0 & 0.34 & 0.0001 \\
\hline 29300 & 0.0073 & 24.21 & 1.8 & 0.26 & 0.0001 \\
\hline 32000 & 0.0067 & 24.27 & 1.5 & 0.26 & 0.0001 \\
\hline 35000 & 0.0061 & 24.33 & 1.3 & 0.26 & 0.0001 \\
\hline 38300 & 0.0056 & 24.40 & 1.0 & 0.26 & 0.0001 \\
\hline 41900 & 0.0051 & 24.44 & 0.9 & 0.17 & 0.0000 \\
\hline 45800 & 0.0047 & 24.50 & 0.6 & 0.26 & 0.0001 \\
\hline 50100 & 0.0043 & 24.54 & 0.4 & 0.17 & 0.0000 \\
\hline 54800 & 0.0039 & 24.58 & 0.3 & 0.17 & 0.0000 \\
\hline 59500 & 0.0036 & 24.64 & 0.0 & 0.26 & 0.0001 \\
\hline
\end{tabular}


Table 10. MICP data for sample 07JRM009 - 19.7a.

\section{Mercury Injection Capillary Pressure 07JRM009 - 19.7a}

\begin{tabular}{|c|c|c|c|c|c|}
\hline \\
\hline \multicolumn{3}{|c|}{$\begin{array}{ll}\text { Bulk Volume }= & 7.6051 \mathrm{cc} \\
\text { Pore Volume }= & 1.6356 \mathrm{cc} \\
\text { Closure }=0.61 \% \mathrm{BV} @ 430 \mathrm{psia} \\
\end{array}$} & \multicolumn{3}{|c|}{$\begin{array}{l}\text { Porosity }=\quad 21.5 \% \text { (mercury) } \\
\text { Permeability }=0.182 \text { md (mercury) } \\
\text { Median Pore Aperture }=0.1960 \text { microns (diameter) }\end{array}$} \\
\hline $\begin{array}{l}\text { Capillary } \\
\text { Pressure } \\
\text { (psia) } \\
\end{array}$ & $\begin{array}{l}\text { Pore Aperture } \\
\text { Diameter } \\
\text { (microns) } \\
\end{array}$ & $\begin{array}{c}\text { Cumulative } \\
\text { Bulk Vol. (\%) } \\
\end{array}$ & $\begin{array}{l}\text { Wetting Phase } \\
\text { Saturation (\%) }\end{array}$ & $\begin{array}{l}\text { Incremental } \\
\text { Wetting Phase } \\
\text { Change (\%) } \\
\end{array}$ & $\begin{array}{l}\text { Saturation } \\
\text { Change } \\
\text { per psia } \\
\end{array}$ \\
\hline 1.64 & 130 & 0.00 & 100.0 & 0.00 & 0.0000 \\
\hline 1.80 & 119 & 0.02 & 100.0 & 0.00 & 0.0000 \\
\hline 1.96 & 109 & 0.02 & 100.0 & 0.00 & 0.0000 \\
\hline 2.15 & 99.2 & 0.02 & 100.0 & 0.00 & 0.0000 \\
\hline 2.35 & 90.8 & 0.02 & 100.0 & 0.00 & 0.0000 \\
\hline 2.57 & 83.0 & 0.02 & 100.0 & 0.00 & 0.0000 \\
\hline 2.81 & 75.9 & 0.02 & 100.0 & 0.00 & 0.0000 \\
\hline 3.08 & 69.3 & 0.02 & 100.0 & 0.00 & 0.0000 \\
\hline 3.37 & 63.3 & 0.04 & 100.0 & 0.00 & 0.0000 \\
\hline 3.68 & 58.0 & 0.04 & 100.0 & 0.00 & 0.0000 \\
\hline 4.03 & 52.9 & 0.08 & 100.0 & 0.00 & 0.0000 \\
\hline 4.41 & 48.4 & 0.08 & 100.0 & 0.00 & 0.0000 \\
\hline 4.82 & 44.3 & 0.11 & 100.0 & 0.00 & 0.0000 \\
\hline 5.27 & 40.5 & 0.11 & 100.0 & 0.00 & 0.0000 \\
\hline 5.77 & 37.0 & 0.11 & 100.0 & 0.00 & 0.0000 \\
\hline 6.31 & 33.8 & 0.13 & 100.0 & 0.00 & 0.0000 \\
\hline 6.90 & 30.9 & 0.13 & 100.0 & 0.00 & 0.0000 \\
\hline 7.55 & 28.3 & 0.13 & 100.0 & 0.00 & 0.0000 \\
\hline 8.26 & 25.8 & 0.15 & 100.0 & 0.00 & 0.0000 \\
\hline 9.04 & 23.6 & 0.15 & 100.0 & 0.00 & 0.0000 \\
\hline 9.89 & 21.6 & 0.15 & 100.0 & 0.00 & 0.0000 \\
\hline 10.8 & 19.8 & 0.15 & 100.0 & 0.00 & 0.0000 \\
\hline 11.8 & 18.1 & 0.15 & 100.0 & 0.00 & 0.0000 \\
\hline 12.9 & 16.5 & 0.17 & 100.0 & 0.00 & 0.0000 \\
\hline 14.2 & 15.0 & 0.17 & 100.0 & 0.00 & 0.0000 \\
\hline 15.5 & 13.8 & 0.17 & 100.0 & 0.00 & 0.0000 \\
\hline 16.9 & 12.6 & 0.17 & 100.0 & 0.00 & 0.0000 \\
\hline 18.5 & 11.5 & 0.19 & 100.0 & 0.00 & 0.0000 \\
\hline 20.3 & 10.5 & 0.19 & 100.0 & 0.00 & 0.0000 \\
\hline 22.2 & 9.61 & 0.21 & 100.0 & 0.00 & 0.0000 \\
\hline 24.3 & 8.78 & 0.21 & 100.0 & 0.00 & 0.0000 \\
\hline 26.6 & 8.02 & 0.21 & 100.0 & 0.00 & 0.0000 \\
\hline 29.0 & 7.36 & 0.23 & 100.0 & 0.00 & 0.0000 \\
\hline 31.8 & 6.71 & 0.23 & 100.0 & 0.00 & 0.0000 \\
\hline 34.8 & 6.13 & 0.25 & 100.0 & 0.00 & 0.0000 \\
\hline 38.0 & 5.61 & 0.27 & 100.0 & 0.00 & 0.0000 \\
\hline
\end{tabular}


Table 10 (cont.). MICP data for sample 07JRM009 - 19.7a.

\section{Mercury Injection Capillary Pressure 07JRM009 - 19.7a}

\begin{tabular}{|c|c|c|c|c|c|}
\hline $\begin{array}{c}\text { Capillary } \\
\text { Pressure } \\
\text { (psia) } \\
\end{array}$ & $\begin{array}{c}\text { Pore Aperture } \\
\text { Diameter } \\
\text { (microns) }\end{array}$ & $\begin{array}{c}\text { Cumulative } \\
\text { Bulk Vol. (\%) } \\
\end{array}$ & $\begin{array}{l}\text { Wetting Phase } \\
\text { Saturation (\%) }\end{array}$ & $\begin{array}{c}\text { Incremental } \\
\text { Wetting Phase } \\
\text { Change (\%) } \\
\end{array}$ & $\begin{array}{c}\text { Saturation } \\
\text { Change } \\
\text { per psia } \\
\end{array}$ \\
\hline 41.6 & 5.13 & 0.29 & 100.0 & 0.00 & 0.0000 \\
\hline 45.5 & 4.69 & 0.32 & 100.0 & 0.00 & 0.0000 \\
\hline 49.8 & 4.28 & 0.32 & 100.0 & 0.00 & 0.0000 \\
\hline 54.5 & 3.91 & 0.32 & 100.0 & 0.00 & 0.0000 \\
\hline 59.6 & 3.58 & 0.32 & 100.0 & 0.00 & 0.0000 \\
\hline 65.2 & 3.27 & 0.34 & 100.0 & 0.00 & 0.0000 \\
\hline 71.3 & 2.99 & 0.34 & 100.0 & 0.00 & 0.0000 \\
\hline 78.0 & 2.73 & 0.34 & 100.0 & 0.00 & 0.0000 \\
\hline 85.3 & 2.50 & 0.34 & 100.0 & 0.00 & 0.0000 \\
\hline 93.4 & 2.28 & 0.34 & 100.0 & 0.00 & 0.0000 \\
\hline 102 & 2.09 & 0.34 & 100.0 & 0.00 & 0.0000 \\
\hline 112 & 1.90 & 0.36 & 100.0 & 0.00 & 0.0000 \\
\hline 122 & 1.75 & 0.36 & 100.0 & 0.00 & 0.0000 \\
\hline 134 & 1.59 & 0.36 & 100.0 & 0.00 & 0.0000 \\
\hline 146 & 1.46 & 0.38 & 100.0 & 0.00 & 0.0000 \\
\hline 160 & 1.33 & 0.38 & 100.0 & 0.00 & 0.0000 \\
\hline 175 & 1.22 & 0.38 & 100.0 & 0.00 & 0.0000 \\
\hline 191 & 1.12 & 0.40 & 100.0 & 0.00 & 0.0000 \\
\hline 209 & 1.02 & 0.40 & 100.0 & 0.00 & 0.0000 \\
\hline 229 & 0.932 & 0.42 & 100.0 & 0.00 & 0.0000 \\
\hline 251 & 0.850 & 0.44 & 100.0 & 0.00 & 0.0000 \\
\hline 274 & 0.779 & 0.46 & 100.0 & 0.00 & 0.0000 \\
\hline 300 & 0.711 & 0.48 & 100.0 & 0.00 & 0.0000 \\
\hline 328 & 0.650 & 0.51 & 100.0 & 0.00 & 0.0000 \\
\hline 359 & 0.594 & 0.55 & 100.0 & 0.00 & 0.0000 \\
\hline 393 & 0.543 & 0.57 & 100.0 & 0.00 & 0.0000 \\
\hline 430 & 0.496 & 0.61 & 100.0 & 0.00 & 0.0000 \\
\hline 470 & 0.454 & 0.67 & 99.7 & 0.29 & 0.0073 \\
\hline 514 & 0.415 & 0.86 & 98.8 & 0.88 & 0.0200 \\
\hline 563 & 0.379 & 1.37 & 96.5 & 2.35 & 0.0479 \\
\hline 615 & 0.347 & 2.57 & 90.9 & 5.58 & 0.1073 \\
\hline 673 & 0.317 & 4.40 & 82.4 & 8.51 & 0.1468 \\
\hline 736 & 0.290 & 6.17 & 74.2 & 8.22 & 0.1305 \\
\hline 806 & 0.265 & 7.83 & 66.4 & 7.73 & 0.1104 \\
\hline 881 & 0.242 & 9.24 & 59.9 & 6.56 & 0.0874 \\
\hline 964 & 0.221 & 10.21 & 55.4 & 4.50 & 0.0542 \\
\hline 1050 & 0.203 & 11.03 & 51.6 & 3.82 & 0.0444 \\
\hline 1150 & 0.186 & 11.85 & 47.7 & 3.82 & 0.0382 \\
\hline 1260 & 0.169 & 12.63 & 44.1 & 3.62 & 0.0329 \\
\hline 1380 & 0.155 & 13.30 & 41.0 & 3.13 & 0.0261 \\
\hline 1510 & 0.141 & 13.91 & 38.2 & 2.84 & 0.0218 \\
\hline 1650 & 0.129 & 14.44 & 35.7 & 2.45 & 0.0175 \\
\hline
\end{tabular}


Table 10 (cont.). MICP data for sample 07JRM009 - 19.7a.

\section{Mercury Injection Capillary Pressure 07JRM009 - 19.7a}

\begin{tabular}{|c|c|c|c|c|c|}
\hline $\begin{array}{c}\text { Capillary } \\
\text { Pressure } \\
\text { (psia) } \\
\end{array}$ & $\begin{array}{c}\text { Pore Aperture } \\
\text { Diameter } \\
\text { (microns) }\end{array}$ & $\begin{array}{c}\text { Cumulative } \\
\text { Bulk Vol. (\%) }\end{array}$ & $\begin{array}{l}\text { Wetting Phase } \\
\text { Saturation (\%) }\end{array}$ & $\begin{array}{c}\text { Incremental } \\
\text { Wetting Phase } \\
\text { Change (\%) }\end{array}$ & $\begin{array}{c}\text { Saturation } \\
\text { Change } \\
\text { per psia }\end{array}$ \\
\hline 1810 & 0.1179 & 14.92 & 33.5 & 2.25 & 0.0141 \\
\hline 1980 & 0.1077 & 15.38 & 31.3 & 2.15 & 0.0127 \\
\hline 2160 & 0.0988 & 15.80 & 29.4 & 1.96 & 0.0109 \\
\hline 2370 & 0.0900 & 16.22 & 27.4 & 1.96 & 0.0093 \\
\hline 2590 & 0.0824 & 16.60 & 25.6 & 1.76 & 0.0080 \\
\hline 2830 & 0.0754 & 16.96 & 24.0 & 1.66 & 0.0069 \\
\hline 3100 & 0.0688 & 17.32 & 22.3 & 1.66 & 0.0062 \\
\hline 3390 & 0.0629 & 17.63 & 20.8 & 1.47 & 0.0051 \\
\hline 3710 & 0.0575 & 17.93 & 19.5 & 1.37 & 0.0043 \\
\hline 4060 & 0.0525 & 18.18 & 18.3 & 1.17 & 0.0034 \\
\hline 4440 & 0.0480 & 18.46 & 17.0 & 1.27 & 0.0033 \\
\hline 4850 & 0.0440 & 18.69 & 15.9 & 1.08 & 0.0026 \\
\hline 5310 & 0.0402 & 18.90 & 15.0 & 0.98 & 0.0021 \\
\hline 5810 & 0.0367 & 19.11 & 14.0 & 0.98 & 0.0020 \\
\hline 6360 & 0.0335 & 19.30 & 13.1 & 0.88 & 0.0016 \\
\hline 6950 & 0.0307 & 19.51 & 12.1 & 0.98 & 0.0017 \\
\hline 7610 & 0.0280 & 19.70 & 11.3 & 0.88 & 0.0013 \\
\hline 8320 & 0.0256 & 19.87 & 10.5 & 0.78 & 0.0011 \\
\hline 9100 & 0.0234 & 20.03 & 9.7 & 0.78 & 0.0010 \\
\hline 9960 & 0.0214 & 20.18 & 9.0 & 0.68 & 0.0008 \\
\hline 10900 & 0.0196 & 20.33 & 8.3 & 0.68 & 0.0007 \\
\hline 11900 & 0.0179 & 20.48 & 7.6 & 0.68 & 0.0007 \\
\hline 13000 & 0.0164 & 20.60 & 7.0 & 0.59 & 0.0005 \\
\hline 14300 & 0.0149 & 20.73 & 6.5 & 0.59 & 0.0005 \\
\hline 15600 & 0.0137 & 20.85 & 5.9 & 0.59 & 0.0005 \\
\hline 17100 & 0.0125 & 20.98 & 5.3 & 0.59 & 0.0004 \\
\hline 18700 & 0.0114 & 21.09 & 4.8 & 0.49 & 0.0003 \\
\hline 20400 & 0.0105 & 21.19 & 4.3 & 0.49 & 0.0003 \\
\hline 22300 & 0.0096 & 21.28 & 3.9 & 0.39 & 0.0002 \\
\hline 24400 & 0.0087 & 21.38 & 3.4 & 0.49 & 0.0002 \\
\hline 26700 & 0.0080 & 21.49 & 2.9 & 0.49 & 0.0002 \\
\hline 29300 & 0.0073 & 21.57 & 2.5 & 0.39 & 0.0002 \\
\hline 32000 & 0.0067 & 21.63 & 2.3 & 0.29 & 0.0001 \\
\hline 35000 & 0.0061 & 21.72 & 1.9 & 0.39 & 0.0001 \\
\hline 38300 & 0.0056 & 21.78 & 1.6 & 0.29 & 0.0001 \\
\hline 41900 & 0.0051 & 21.86 & 1.2 & 0.39 & 0.0001 \\
\hline 45800 & 0.0047 & 21.91 & 1.0 & 0.20 & 0.0001 \\
\hline 50100 & 0.0043 & 21.97 & 0.7 & 0.29 & 0.0001 \\
\hline 54800 & 0.0039 & 22.05 & 0.3 & 0.39 & 0.0001 \\
\hline 59500 & 0.0036 & 22.12 & 0.0 & 0.29 & 0.0001 \\
\hline
\end{tabular}


Table 11. MICP data for sample 07JRM010 - 34.9a.

\section{Mercury Injection Capillary Pressure 07JRM010 - 34.9a}

\begin{tabular}{|c|c|c|c|c|c|}
\hline \multicolumn{3}{|c|}{\begin{tabular}{ll}
\multicolumn{2}{l}{ Sample Information } \\
\cline { 1 - 2 } Bulk Volume $=$ & $5.7696 \mathrm{cc}$ \\
Pore Volume $=$ & $1.3428 \mathrm{cc}$ \\
Closure = 0.69 \%BV @ $673 \mathrm{psia}$
\end{tabular}} & \multicolumn{3}{|c|}{$\begin{array}{ll}\text { Porosity }= & 23.3 \% \text { (mercury) } \\
\text { Permeability }= & 0.0901 \text { md (mercury) } \\
\text { Median Pore Aperture }=0.1188 \text { microns (diameter) }\end{array}$} \\
\hline $\begin{array}{l}\text { Capillary } \\
\text { Pressure } \\
\text { (psia) } \\
\end{array}$ & $\begin{array}{c}\text { Pore Aperture } \\
\text { Diameter } \\
\text { (microns) } \\
\end{array}$ & $\begin{array}{l}\text { Cumulative } \\
\text { Bulk Vol. (\%) } \\
\end{array}$ & $\begin{array}{l}\text { Wetting Phase } \\
\text { Saturation (\%) }\end{array}$ & $\begin{array}{l}\text { Incremental } \\
\text { Wetting Phase } \\
\text { Change (\%) } \\
\end{array}$ & $\begin{array}{c}\text { Saturation } \\
\text { Change } \\
\text { per psia } \\
\end{array}$ \\
\hline 1.64 & 130 & 0.00 & 100.0 & 0.00 & 0.0000 \\
\hline 1.80 & 119 & 0.00 & 100.0 & 0.00 & 0.0000 \\
\hline 1.96 & 109 & 0.02 & 100.0 & 0.00 & 0.0000 \\
\hline 2.15 & 99.2 & 0.02 & 100.0 & 0.00 & 0.0000 \\
\hline 2.35 & 90.8 & 0.02 & 100.0 & 0.00 & 0.0000 \\
\hline 2.57 & 83.0 & 0.02 & 100.0 & 0.00 & 0.0000 \\
\hline 2.81 & 75.9 & 0.02 & 100.0 & 0.00 & 0.0000 \\
\hline 3.08 & 69.3 & 0.02 & 100.0 & 0.00 & 0.0000 \\
\hline 3.37 & 63.3 & 0.04 & 100.0 & 0.00 & 0.0000 \\
\hline 3.68 & 58.0 & 0.04 & 100.0 & 0.00 & 0.0000 \\
\hline 4.03 & 52.9 & 0.04 & 100.0 & 0.00 & 0.0000 \\
\hline 4.41 & 48.4 & 0.04 & 100.0 & 0.00 & 0.0000 \\
\hline 4.82 & 44.3 & 0.04 & 100.0 & 0.00 & 0.0000 \\
\hline 5.27 & 40.5 & 0.04 & 100.0 & 0.00 & 0.0000 \\
\hline 5.77 & 37.0 & 0.06 & 100.0 & 0.00 & 0.0000 \\
\hline 6.31 & 33.8 & 0.06 & 100.0 & 0.00 & 0.0000 \\
\hline 6.90 & 30.9 & 0.06 & 100.0 & 0.00 & 0.0000 \\
\hline 7.55 & 28.3 & 0.08 & 100.0 & 0.00 & 0.0000 \\
\hline 8.26 & 25.8 & 0.08 & 100.0 & 0.00 & 0.0000 \\
\hline 9.04 & 23.6 & 0.10 & 100.0 & 0.00 & 0.0000 \\
\hline 9.89 & 21.6 & 0.10 & 100.0 & 0.00 & 0.0000 \\
\hline 10.8 & 19.8 & 0.10 & 100.0 & 0.00 & 0.0000 \\
\hline 11.8 & 18.1 & 0.12 & 100.0 & 0.00 & 0.0000 \\
\hline 12.9 & 16.5 & 0.12 & 100.0 & 0.00 & 0.0000 \\
\hline 14.2 & 15.0 & 0.12 & 100.0 & 0.00 & 0.0000 \\
\hline 15.5 & 13.8 & 0.14 & 100.0 & 0.00 & 0.0000 \\
\hline 16.9 & 12.6 & 0.14 & 100.0 & 0.00 & 0.0000 \\
\hline 18.5 & 11.5 & 0.16 & 100.0 & 0.00 & 0.0000 \\
\hline 20.3 & 10.5 & 0.16 & 100.0 & 0.00 & 0.0000 \\
\hline 22.2 & 9.61 & 0.16 & 100.0 & 0.00 & 0.0000 \\
\hline 24.3 & 8.78 & 0.18 & 100.0 & 0.00 & 0.0000 \\
\hline 26.6 & 8.02 & 0.18 & 100.0 & 0.00 & 0.0000 \\
\hline 29.0 & 7.36 & 0.20 & 100.0 & 0.00 & 0.0000 \\
\hline 31.8 & 6.71 & 0.20 & 100.0 & 0.00 & 0.0000 \\
\hline 34.8 & 6.13 & 0.22 & 100.0 & 0.00 & 0.0000 \\
\hline 38.0 & 5.61 & 0.24 & 100.0 & 0.00 & 0.0000 \\
\hline
\end{tabular}


Table 11 (cont.). MICP data for sample 07JRM010 - 34.9a.

\section{Mercury Injection Capillary Pressure 07JRM010 - 34.9a}

\begin{tabular}{|c|c|c|c|c|c|}
\hline $\begin{array}{c}\text { Capillary } \\
\text { Pressure } \\
\text { (psia) } \\
\end{array}$ & $\begin{array}{l}\text { Pore Aperture } \\
\text { Diameter } \\
\text { (microns) } \\
\end{array}$ & $\begin{array}{c}\text { Cumulative } \\
\text { Bulk Vol. (\%) } \\
\end{array}$ & $\begin{array}{l}\text { Wetting Phase } \\
\text { Saturation (\%) }\end{array}$ & $\begin{array}{c}\text { Incremental } \\
\text { Wetting Phase } \\
\text { Change (\%) } \\
\end{array}$ & $\begin{array}{c}\text { Saturation } \\
\text { Change } \\
\text { per psia } \\
\end{array}$ \\
\hline 41.6 & 5.13 & 0.26 & 100.0 & 0.00 & 0.0000 \\
\hline 45.5 & 4.69 & 0.28 & 100.0 & 0.00 & 0.0000 \\
\hline 49.8 & 4.28 & 0.28 & 100.0 & 0.00 & 0.0000 \\
\hline 54.5 & 3.91 & 0.28 & 100.0 & 0.00 & 0.0000 \\
\hline 59.6 & 3.58 & 0.28 & 100.0 & 0.00 & 0.0000 \\
\hline 65.2 & 3.27 & 0.28 & 100.0 & 0.00 & 0.0000 \\
\hline 71.3 & 2.99 & 0.28 & 100.0 & 0.00 & 0.0000 \\
\hline 78.0 & 2.73 & 0.30 & 100.0 & 0.00 & 0.0000 \\
\hline 85.3 & 2.50 & 0.30 & 100.0 & 0.00 & 0.0000 \\
\hline 93.4 & 2.28 & 0.30 & 100.0 & 0.00 & 0.0000 \\
\hline 102 & 2.09 & 0.30 & 100.0 & 0.00 & 0.0000 \\
\hline 112 & 1.90 & 0.32 & 100.0 & 0.00 & 0.0000 \\
\hline 122 & 1.75 & 0.32 & 100.0 & 0.00 & 0.0000 \\
\hline 134 & 1.59 & 0.32 & 100.0 & 0.00 & 0.0000 \\
\hline 146 & 1.46 & 0.34 & 100.0 & 0.00 & 0.0000 \\
\hline 160 & 1.33 & 0.34 & 100.0 & 0.00 & 0.0000 \\
\hline 175 & 1.22 & 0.34 & 100.0 & 0.00 & 0.0000 \\
\hline 191 & 1.12 & 0.36 & 100.0 & 0.00 & 0.0000 \\
\hline 209 & 1.02 & 0.36 & 100.0 & 0.00 & 0.0000 \\
\hline 229 & 0.932 & 0.38 & 100.0 & 0.00 & 0.0000 \\
\hline 251 & 0.850 & 0.38 & 100.0 & 0.00 & 0.0000 \\
\hline 274 & 0.779 & 0.40 & 100.0 & 0.00 & 0.0000 \\
\hline 300 & 0.711 & 0.42 & 100.0 & 0.00 & 0.0000 \\
\hline 328 & 0.650 & 0.42 & 100.0 & 0.00 & 0.0000 \\
\hline 359 & 0.594 & 0.44 & 100.0 & 0.00 & 0.0000 \\
\hline 393 & 0.543 & 0.47 & 100.0 & 0.00 & 0.0000 \\
\hline 430 & 0.496 & 0.49 & 100.0 & 0.00 & 0.0000 \\
\hline 470 & 0.454 & 0.53 & 100.0 & 0.00 & 0.0000 \\
\hline 514 & 0.415 & 0.55 & 100.0 & 0.00 & 0.0000 \\
\hline 563 & 0.379 & 0.59 & 100.0 & 0.00 & 0.0000 \\
\hline 615 & 0.347 & 0.63 & 100.0 & 0.00 & 0.0000 \\
\hline 673 & 0.317 & 0.69 & 100.0 & 0.00 & 0.0000 \\
\hline 736 & 0.290 & 0.77 & 99.7 & 0.35 & 0.0055 \\
\hline 806 & 0.265 & 0.97 & 98.8 & 0.87 & 0.0124 \\
\hline 881 & 0.242 & 1.42 & 96.9 & 1.91 & 0.0255 \\
\hline 964 & 0.221 & 2.85 & 90.7 & 6.17 & 0.0744 \\
\hline 1050 & 0.203 & 4.31 & 84.4 & 6.26 & 0.0728 \\
\hline 1150 & 0.186 & 6.51 & 75.0 & 9.48 & 0.0948 \\
\hline 1260 & 0.169 & 8.33 & 67.1 & 7.83 & 0.0711 \\
\hline 1380 & 0.155 & 9.50 & 62.1 & 5.04 & 0.0420 \\
\hline 1510 & 0.141 & 10.53 & 57.7 & 4.43 & 0.0341 \\
\hline 1650 & 0.129 & 11.48 & 53.6 & 4.09 & 0.0292 \\
\hline
\end{tabular}


Table 11 (cont.). MICP data for sample 07JRM010 - 34.9a.

\section{Mercury Injection Capillary Pressure 07JRM010 - 34.9a}

\begin{tabular}{|c|c|c|c|c|c|}
\hline $\begin{array}{c}\text { Capillary } \\
\text { Pressure } \\
\text { (psia) } \\
\end{array}$ & $\begin{array}{l}\text { Pore Aperture } \\
\text { Diameter } \\
\text { (microns) } \\
\end{array}$ & $\begin{array}{c}\text { Cumulative } \\
\text { Bulk Vol. (\%) }\end{array}$ & $\begin{array}{l}\text { Wetting Phase } \\
\text { Saturation (\%) }\end{array}$ & $\begin{array}{c}\text { Incremental } \\
\text { Wetting Phase } \\
\text { Change (\%) }\end{array}$ & $\begin{array}{c}\text { Saturation } \\
\text { Change } \\
\text { per psia }\end{array}$ \\
\hline 1810 & 0.1179 & 12.39 & 49.7 & 3.91 & 0.0245 \\
\hline 1980 & 0.1077 & 13.22 & 46.1 & 3.57 & 0.0210 \\
\hline 2160 & 0.0988 & 13.97 & 42.9 & 3.22 & 0.0179 \\
\hline 2370 & 0.0900 & 14.70 & 39.7 & 3.13 & 0.0149 \\
\hline 2590 & 0.0824 & 15.35 & 37.0 & 2.78 & 0.0126 \\
\hline 2830 & 0.0754 & 15.97 & 34.3 & 2.70 & 0.0112 \\
\hline 3100 & 0.0688 & 16.58 & 31.7 & 2.61 & 0.0097 \\
\hline 3390 & 0.0629 & 17.13 & 29.3 & 2.35 & 0.0081 \\
\hline 3710 & 0.0575 & 17.63 & 27.1 & 2.17 & 0.0068 \\
\hline 4060 & 0.0525 & 18.12 & 25.0 & 2.09 & 0.0060 \\
\hline 4440 & 0.0480 & 18.56 & 23.1 & 1.91 & 0.0050 \\
\hline 4850 & 0.0440 & 18.93 & 21.6 & 1.57 & 0.0038 \\
\hline 5310 & 0.0402 & 19.31 & 19.9 & 1.65 & 0.0036 \\
\hline 5810 & 0.0367 & 19.67 & 18.3 & 1.57 & 0.0031 \\
\hline 6360 & 0.0335 & 20.00 & 17.0 & 1.39 & 0.0025 \\
\hline 6950 & 0.0307 & 20.30 & 15.7 & 1.30 & 0.0022 \\
\hline 7610 & 0.0280 & 20.60 & 14.3 & 1.30 & 0.0020 \\
\hline 8320 & 0.0256 & 20.87 & 13.2 & 1.13 & 0.0016 \\
\hline 9100 & 0.0234 & 21.11 & 12.2 & 1.04 & 0.0013 \\
\hline 9960 & 0.0214 & 21.33 & 11.2 & 0.96 & 0.0011 \\
\hline 10900 & 0.0196 & 21.55 & 10.3 & 0.96 & 0.0010 \\
\hline 11900 & 0.0179 & 21.76 & 9.4 & 0.87 & 0.0009 \\
\hline 13000 & 0.0164 & 21.94 & 8.6 & 0.78 & 0.0007 \\
\hline 14300 & 0.0149 & 22.12 & 7.8 & 0.78 & 0.0006 \\
\hline 15600 & 0.0137 & 22.28 & 7.1 & 0.70 & 0.0005 \\
\hline 17100 & 0.0125 & 22.44 & 6.4 & 0.70 & 0.0005 \\
\hline 18700 & 0.0114 & 22.59 & 5.8 & 0.61 & 0.0004 \\
\hline 20400 & 0.0105 & 22.73 & 5.2 & 0.61 & 0.0004 \\
\hline 22300 & 0.0096 & 22.87 & 4.6 & 0.61 & 0.0003 \\
\hline 24400 & 0.0087 & 22.99 & 4.1 & 0.52 & 0.0002 \\
\hline 26700 & 0.0080 & 23.11 & 3.6 & 0.52 & 0.0002 \\
\hline 29300 & 0.0073 & 23.23 & 3.0 & 0.52 & 0.0002 \\
\hline 32000 & 0.0067 & 23.33 & 2.6 & 0.43 & 0.0002 \\
\hline 35000 & 0.0061 & 23.43 & 2.2 & 0.43 & 0.0001 \\
\hline 38300 & 0.0056 & 23.54 & 1.7 & 0.43 & 0.0001 \\
\hline 41900 & 0.0051 & 23.62 & 1.4 & 0.35 & 0.0001 \\
\hline 45800 & 0.0047 & 23.70 & 1.0 & 0.35 & 0.0001 \\
\hline 50100 & 0.0043 & 23.78 & 0.7 & 0.35 & 0.0001 \\
\hline 54800 & 0.0039 & 23.84 & 0.4 & 0.26 & 0.0001 \\
\hline 59500 & 0.0036 & 23.94 & 0.0 & 0.43 & 0.0001 \\
\hline
\end{tabular}


Table 12. MICP data for sample 07JRM011 - 24.7a.

\section{Mercury Injection Capillary Pressure 07JRM011 - 24.7a}

\begin{tabular}{|c|c|c|c|c|c|}
\hline \multicolumn{3}{|c|}{\begin{tabular}{ll}
\multicolumn{2}{l}{ Sample Information } \\
\cline { 1 - 2 } Bulk Volume $=$ & $8.1398 \mathrm{cc}$ \\
Pore Volume $=$ & $2.7671 \mathrm{cc}$ \\
Closure $=0.44 \% \mathrm{BV} @ 359 \mathrm{psia}$ \\
\end{tabular}} & \multicolumn{3}{|c|}{$\begin{array}{lc}\text { Porosity }= & 34.0 \% \text { (mercury) } \\
\text { Permeability }= & 0.150 \mathrm{md} \text { (mercury) } \\
\text { Median Pore Aperture }=0.1117 \text { microns (diameter) }\end{array}$} \\
\hline $\begin{array}{l}\text { Capillary } \\
\text { Pressure } \\
\text { (psia) } \\
\end{array}$ & $\begin{array}{l}\text { Pore Aperture } \\
\text { Diameter } \\
\text { (microns) } \\
\end{array}$ & $\begin{array}{l}\text { Cumulative } \\
\text { Bulk Vol. (\%) } \\
\end{array}$ & $\begin{array}{l}\text { Wetting Phase } \\
\text { Saturation (\%) }\end{array}$ & $\begin{array}{l}\text { Incremental } \\
\text { Wetting Phase } \\
\text { Change (\%) } \\
\end{array}$ & $\begin{array}{l}\text { Saturation } \\
\text { Change } \\
\text { per psia }\end{array}$ \\
\hline 1.64 & 130 & 0.00 & 100.0 & 0.00 & 0.0000 \\
\hline 1.80 & 119 & 0.02 & 100.0 & 0.00 & 0.0000 \\
\hline 1.96 & 109 & 0.02 & 100.0 & 0.00 & 0.0000 \\
\hline 2.15 & 99.2 & 0.02 & 100.0 & 0.00 & 0.0000 \\
\hline 2.35 & 90.8 & 0.04 & 100.0 & 0.00 & 0.0000 \\
\hline 2.57 & 83.0 & 0.04 & 100.0 & 0.00 & 0.0000 \\
\hline 2.81 & 75.9 & 0.04 & 100.0 & 0.00 & 0.0000 \\
\hline 3.08 & 69.3 & 0.04 & 100.0 & 0.00 & 0.0000 \\
\hline 3.37 & 63.3 & 0.04 & 100.0 & 0.00 & 0.0000 \\
\hline 3.68 & 58.0 & 0.04 & 100.0 & 0.00 & 0.0000 \\
\hline 4.03 & 52.9 & 0.06 & 100.0 & 0.00 & 0.0000 \\
\hline 4.41 & 48.4 & 0.06 & 100.0 & 0.00 & 0.0000 \\
\hline 4.82 & 44.3 & 0.06 & 100.0 & 0.00 & 0.0000 \\
\hline 5.27 & 40.5 & 0.06 & 100.0 & 0.00 & 0.0000 \\
\hline 5.77 & 37.0 & 0.06 & 100.0 & 0.00 & 0.0000 \\
\hline 6.31 & 33.8 & 0.06 & 100.0 & 0.00 & 0.0000 \\
\hline 6.90 & 30.9 & 0.06 & 100.0 & 0.00 & 0.0000 \\
\hline 7.55 & 28.3 & 0.06 & 100.0 & 0.00 & 0.0000 \\
\hline 8.26 & 25.8 & 0.06 & 100.0 & 0.00 & 0.0000 \\
\hline 9.04 & 23.6 & 0.06 & 100.0 & 0.00 & 0.0000 \\
\hline 9.89 & 21.6 & 0.06 & 100.0 & 0.00 & 0.0000 \\
\hline 10.8 & 19.8 & 0.09 & 100.0 & 0.00 & 0.0000 \\
\hline 11.8 & 18.1 & 0.11 & 100.0 & 0.00 & 0.0000 \\
\hline 12.9 & 16.5 & 0.11 & 100.0 & 0.00 & 0.0000 \\
\hline 14.2 & 15.0 & 0.11 & 100.0 & 0.00 & 0.0000 \\
\hline 15.5 & 13.8 & 0.13 & 100.0 & 0.00 & 0.0000 \\
\hline 16.9 & 12.6 & 0.13 & 100.0 & 0.00 & 0.0000 \\
\hline 18.5 & 11.5 & 0.13 & 100.0 & 0.00 & 0.0000 \\
\hline 20.3 & 10.5 & 0.13 & 100.0 & 0.00 & 0.0000 \\
\hline 22.2 & 9.61 & 0.15 & 100.0 & 0.00 & 0.0000 \\
\hline 24.3 & 8.78 & 0.15 & 100.0 & 0.00 & 0.0000 \\
\hline 26.6 & 8.02 & 0.15 & 100.0 & 0.00 & 0.0000 \\
\hline 29.0 & 7.36 & 0.17 & 100.0 & 0.00 & 0.0000 \\
\hline 31.8 & 6.71 & 0.17 & 100.0 & 0.00 & 0.0000 \\
\hline 34.8 & 6.13 & 0.19 & 100.0 & 0.00 & 0.0000 \\
\hline 38.0 & 5.61 & 0.19 & 100.0 & 0.00 & 0.0000 \\
\hline
\end{tabular}


Table 12 (cont.). MICP data for sample 07JRM011 - 24.7a.

\section{Mercury Injection Capillary Pressure 07JRM011 - 24.7a}

\begin{tabular}{|c|c|c|c|c|c|}
\hline $\begin{array}{l}\text { Capillary } \\
\text { Pressure } \\
\text { (psia) } \\
\end{array}$ & $\begin{array}{c}\text { Pore Aperture } \\
\text { Diameter } \\
\text { (microns) }\end{array}$ & $\begin{array}{l}\text { Cumulative } \\
\text { Bulk Vol. (\%) }\end{array}$ & $\begin{array}{l}\text { Wetting Phase } \\
\text { Saturation (\%) }\end{array}$ & $\begin{array}{c}\text { Incremental } \\
\text { Wetting Phase } \\
\text { Change (\%) }\end{array}$ & $\begin{array}{c}\text { Saturation } \\
\text { Change } \\
\text { per psia } \\
\end{array}$ \\
\hline 41.6 & 5.13 & 0.19 & 100.0 & 0.00 & 0.0000 \\
\hline 45.5 & 4.69 & 0.21 & 100.0 & 0.00 & 0.0000 \\
\hline 49.8 & 4.28 & 0.21 & 100.0 & 0.00 & 0.0000 \\
\hline 54.5 & 3.91 & 0.21 & 100.0 & 0.00 & 0.0000 \\
\hline 59.6 & 3.58 & 0.21 & 100.0 & 0.00 & 0.0000 \\
\hline 65.2 & 3.27 & 0.23 & 100.0 & 0.00 & 0.0000 \\
\hline 71.3 & 2.99 & 0.23 & 100.0 & 0.00 & 0.0000 \\
\hline 78.0 & 2.73 & 0.23 & 100.0 & 0.00 & 0.0000 \\
\hline 85.3 & 2.50 & 0.23 & 100.0 & 0.00 & 0.0000 \\
\hline 93.4 & 2.28 & 0.23 & 100.0 & 0.00 & 0.0000 \\
\hline 102 & 2.09 & 0.23 & 100.0 & 0.00 & 0.0000 \\
\hline 112 & 1.90 & 0.25 & 100.0 & 0.00 & 0.0000 \\
\hline 122 & 1.75 & 0.25 & 100.0 & 0.00 & 0.0000 \\
\hline 134 & 1.59 & 0.25 & 100.0 & 0.00 & 0.0000 \\
\hline 146 & 1.46 & 0.27 & 100.0 & 0.00 & 0.0000 \\
\hline 160 & 1.33 & 0.27 & 100.0 & 0.00 & 0.0000 \\
\hline 175 & 1.22 & 0.27 & 100.0 & 0.00 & 0.0000 \\
\hline 191 & 1.12 & 0.28 & 100.0 & 0.00 & 0.0000 \\
\hline 209 & 1.02 & 0.28 & 100.0 & 0.00 & 0.0000 \\
\hline 229 & 0.932 & 0.30 & 100.0 & 0.00 & 0.0000 \\
\hline 251 & 0.850 & 0.30 & 100.0 & 0.00 & 0.0000 \\
\hline 274 & 0.779 & 0.32 & 100.0 & 0.00 & 0.0000 \\
\hline 300 & 0.711 & 0.34 & 100.0 & 0.00 & 0.0000 \\
\hline 328 & 0.650 & 0.38 & 100.0 & 0.00 & 0.0000 \\
\hline 359 & 0.594 & 0.44 & 100.0 & 0.00 & 0.0000 \\
\hline 393 & 0.543 & 0.51 & 99.8 & 0.22 & 0.0066 \\
\hline 430 & 0.496 & 0.64 & 99.4 & 0.39 & 0.0106 \\
\hline 470 & 0.454 & 0.83 & 98.8 & 0.56 & 0.0140 \\
\hline 514 & 0.415 & 1.19 & 97.8 & 1.06 & 0.0241 \\
\hline 563 & 0.379 & 1.63 & 96.5 & 1.28 & 0.0262 \\
\hline 615 & 0.347 & 2.60 & 93.6 & 2.85 & 0.0547 \\
\hline 673 & 0.317 & 3.32 & 91.5 & 2.12 & 0.0366 \\
\hline 736 & 0.290 & 4.24 & 88.8 & 2.73 & 0.0434 \\
\hline 806 & 0.265 & 5.08 & 86.3 & 2.46 & 0.0351 \\
\hline 881 & 0.242 & 5.91 & 83.9 & 2.46 & 0.0327 \\
\hline 964 & 0.221 & 6.90 & 81.0 & 2.90 & 0.0350 \\
\hline 1050 & 0.203 & 7.96 & 77.8 & 3.13 & 0.0363 \\
\hline 1150 & 0.186 & 9.42 & 73.5 & 4.30 & 0.0430 \\
\hline 1260 & 0.169 & 11.09 & 68.6 & 4.91 & 0.0446 \\
\hline 1380 & 0.155 & 12.79 & 63.6 & 5.02 & 0.0419 \\
\hline 1510 & 0.141 & 14.27 & 59.3 & 4.35 & 0.0335 \\
\hline 1650 & 0.129 & 15.56 & 55.5 & 3.79 & 0.0271 \\
\hline
\end{tabular}


Table 12 (cont.). MICP data for sample 07JRM011 - 24.7a.

\section{Mercury Injection Capillary Pressure 07JRM011 - 24.7a}

\begin{tabular}{|c|c|c|c|c|c|}
\hline $\begin{array}{c}\text { Capillary } \\
\text { Pressure } \\
\text { (psia) } \\
\end{array}$ & $\begin{array}{c}\text { Pore Aperture } \\
\text { Diameter } \\
\text { (microns) }\end{array}$ & $\begin{array}{c}\text { Cumulative } \\
\text { Bulk Vol. (\%) }\end{array}$ & $\begin{array}{l}\text { Wetting Phase } \\
\text { Saturation (\%) }\end{array}$ & $\begin{array}{c}\text { Incremental } \\
\text { Wetting Phase } \\
\text { Change (\%) }\end{array}$ & $\begin{array}{c}\text { Saturation } \\
\text { Change } \\
\text { per psia }\end{array}$ \\
\hline 1810 & 0.1179 & 16.73 & 52.0 & 3.46 & 0.0216 \\
\hline 1980 & 0.1077 & 17.87 & 48.7 & 3.35 & 0.0197 \\
\hline 2160 & 0.0988 & 18.95 & 45.5 & 3.18 & 0.0177 \\
\hline 2370 & 0.0900 & 20.07 & 42.2 & 3.29 & 0.0157 \\
\hline 2590 & 0.0824 & 21.13 & 39.1 & 3.13 & 0.0142 \\
\hline 2830 & 0.0754 & 22.15 & 36.0 & 3.01 & 0.0126 \\
\hline 3100 & 0.0688 & 23.14 & 33.1 & 2.90 & 0.0107 \\
\hline 3390 & 0.0629 & 24.12 & 30.2 & 2.90 & 0.0100 \\
\hline 3710 & 0.0575 & 25.05 & 27.5 & 2.73 & 0.0085 \\
\hline 4060 & 0.0525 & 25.92 & 24.9 & 2.57 & 0.0073 \\
\hline 4440 & 0.0480 & 26.76 & 22.5 & 2.46 & 0.0065 \\
\hline 4850 & 0.0440 & 27.42 & 20.5 & 1.95 & 0.0048 \\
\hline 5310 & 0.0402 & 28.10 & 18.5 & 2.01 & 0.0044 \\
\hline 5810 & 0.0367 & 28.75 & 16.6 & 1.90 & 0.0038 \\
\hline 6360 & 0.0335 & 29.22 & 15.2 & 1.40 & 0.0025 \\
\hline 6950 & 0.0307 & 29.67 & 13.9 & 1.34 & 0.0023 \\
\hline 7610 & 0.0280 & 30.09 & 12.7 & 1.23 & 0.0019 \\
\hline 8320 & 0.0256 & 30.43 & 11.7 & 1.00 & 0.0014 \\
\hline 9100 & 0.0234 & 30.75 & 10.7 & 0.95 & 0.0012 \\
\hline 9960 & 0.0214 & 31.04 & 9.9 & 0.84 & 0.0010 \\
\hline 10900 & 0.0196 & 31.28 & 9.2 & 0.73 & 0.0008 \\
\hline 11900 & 0.0179 & 31.53 & 8.4 & 0.73 & 0.0007 \\
\hline 13000 & 0.0164 & 31.74 & 7.8 & 0.61 & 0.0006 \\
\hline 14300 & 0.0149 & 31.97 & 7.1 & 0.67 & 0.0005 \\
\hline 15600 & 0.0137 & 32.16 & 6.6 & 0.56 & 0.0004 \\
\hline 17100 & 0.0125 & 32.35 & 6.0 & 0.56 & 0.0004 \\
\hline 18700 & 0.0114 & 32.52 & 5.5 & 0.50 & 0.0003 \\
\hline 20400 & 0.0105 & 32.69 & 5.0 & 0.50 & 0.0003 \\
\hline 22300 & 0.0096 & 32.86 & 4.5 & 0.50 & 0.0003 \\
\hline 24400 & 0.0087 & 33.03 & 4.0 & 0.50 & 0.0002 \\
\hline 26700 & 0.0080 & 33.18 & 3.6 & 0.45 & 0.0002 \\
\hline 29300 & 0.0073 & 33.33 & 3.1 & 0.45 & 0.0002 \\
\hline 32000 & 0.0067 & 33.48 & 2.7 & 0.45 & 0.0002 \\
\hline 35000 & 0.0061 & 33.63 & 2.2 & 0.45 & 0.0001 \\
\hline 38300 & 0.0056 & 33.77 & 1.8 & 0.39 & 0.0001 \\
\hline 41900 & 0.0051 & 33.88 & 1.5 & 0.33 & 0.0001 \\
\hline 45800 & 0.0047 & 34.01 & 1.1 & 0.39 & 0.0001 \\
\hline 50100 & 0.0043 & 34.13 & 0.8 & 0.33 & 0.0001 \\
\hline 54800 & 0.0039 & 34.24 & 0.4 & 0.33 & 0.0001 \\
\hline 59500 & 0.0036 & 34.39 & 0.0 & 0.45 & 0.0001 \\
\hline
\end{tabular}


Table 13. MICP data for sample 07DL002 - 17.2a.

\section{Mercury Injection Capillary Pressure 07DL002 - 17.2a}

\begin{tabular}{|c|c|c|c|c|c|}
\hline \multicolumn{3}{|c|}{ 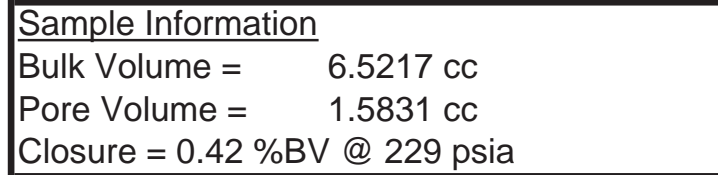 } & \multicolumn{3}{|c|}{$\begin{array}{lc}\text { Porosity }= & 24.3 \% \text { (mercury) } \\
\text { Permeability }= & 0.137 \text { md (mercury) } \\
\text { Median Pore Aperture }=0.1452 \text { microns (diameter) }\end{array}$} \\
\hline $\begin{array}{l}\text { Capillary } \\
\text { Pressure } \\
\text { (psia) }\end{array}$ & $\begin{array}{l}\text { Pore Aperture } \\
\text { Diameter } \\
\text { (microns) }\end{array}$ & $\begin{array}{l}\text { Cumulative } \\
\text { Bulk Vol. (\%) }\end{array}$ & $\begin{array}{l}\text { Wetting Phase } \\
\text { Saturation (\%) }\end{array}$ & $\begin{array}{l}\text { Incremental } \\
\text { Wetting Phase } \\
\text { Change (\%) }\end{array}$ & $\begin{array}{l}\text { Saturation } \\
\text { Change } \\
\text { per psia }\end{array}$ \\
\hline 1.64 & 130 & 0.00 & 100.0 & 0.00 & 0.0000 \\
\hline 1.80 & 119 & 0.02 & 100.0 & 0.00 & 0.0000 \\
\hline 1.96 & 109 & 0.02 & 100.0 & 0.00 & 0.0000 \\
\hline 2.15 & 99.2 & 0.02 & 100.0 & 0.00 & 0.0000 \\
\hline 2.35 & 90.8 & 0.02 & 100.0 & 0.00 & 0.0000 \\
\hline 2.57 & 83.0 & 0.04 & 100.0 & 0.00 & 0.0000 \\
\hline 2.81 & 75.9 & 0.04 & 100.0 & 0.00 & 0.0000 \\
\hline 3.08 & 69.3 & 0.04 & 100.0 & 0.00 & 0.0000 \\
\hline 3.37 & 63.3 & 0.04 & 100.0 & 0.00 & 0.0000 \\
\hline 3.68 & 58.0 & 0.04 & 100.0 & 0.00 & 0.0000 \\
\hline 4.03 & 52.9 & 0.06 & 100.0 & 0.00 & 0.0000 \\
\hline 4.41 & 48.4 & 0.06 & 100.0 & 0.00 & 0.0000 \\
\hline 4.82 & 44.3 & 0.06 & 100.0 & 0.00 & 0.0000 \\
\hline 5.27 & 40.5 & 0.06 & 100.0 & 0.00 & 0.0000 \\
\hline 5.77 & 37.0 & 0.06 & 100.0 & 0.00 & 0.0000 \\
\hline 6.31 & 33.8 & 0.08 & 100.0 & 0.00 & 0.0000 \\
\hline 6.90 & 30.9 & 0.08 & 100.0 & 0.00 & 0.0000 \\
\hline 7.55 & 28.3 & 0.08 & 100.0 & 0.00 & 0.0000 \\
\hline 8.26 & 25.8 & 0.08 & 100.0 & 0.00 & 0.0000 \\
\hline 9.04 & 23.6 & 0.08 & 100.0 & 0.00 & 0.0000 \\
\hline 9.89 & 21.6 & 0.10 & 100.0 & 0.00 & 0.0000 \\
\hline 10.8 & 19.8 & 0.10 & 100.0 & 0.00 & 0.0000 \\
\hline 11.8 & 18.1 & 0.10 & 100.0 & 0.00 & 0.0000 \\
\hline 12.9 & 16.5 & 0.10 & 100.0 & 0.00 & 0.0000 \\
\hline 14.2 & 15.0 & 0.10 & 100.0 & 0.00 & 0.0000 \\
\hline 15.5 & 13.8 & 0.12 & 100.0 & 0.00 & 0.0000 \\
\hline 16.9 & 12.6 & 0.12 & 100.0 & 0.00 & 0.0000 \\
\hline 18.5 & 11.5 & 0.12 & 100.0 & 0.00 & 0.0000 \\
\hline 20.3 & 10.5 & 0.12 & 100.0 & 0.00 & 0.0000 \\
\hline 22.2 & 9.61 & 0.14 & 100.0 & 0.00 & 0.0000 \\
\hline 24.3 & 8.78 & 0.14 & 100.0 & 0.00 & 0.0000 \\
\hline 26.6 & 8.02 & 0.14 & 100.0 & 0.00 & 0.0000 \\
\hline 29.0 & 7.36 & 0.14 & 100.0 & 0.00 & 0.0000 \\
\hline 31.8 & 6.71 & 0.16 & 100.0 & 0.00 & 0.0000 \\
\hline 34.8 & 6.13 & 0.16 & 100.0 & 0.00 & 0.0000 \\
\hline 38.0 & 5.61 & 0.18 & 100.0 & 0.00 & 0.0000 \\
\hline
\end{tabular}


Table 13 (cont.). MICP data for sample 07DL002 - 17.2a.

\section{Mercury Injection Capillary Pressure 07DL002 - 17.2a}

\begin{tabular}{|c|c|c|c|c|c|}
\hline $\begin{array}{c}\text { Capillary } \\
\text { Pressure } \\
\text { (psia) } \\
\end{array}$ & $\begin{array}{c}\text { Pore Aperture } \\
\text { Diameter } \\
\text { (microns) } \\
\end{array}$ & $\begin{array}{l}\text { Cumulative } \\
\text { Bulk Vol. (\%) }\end{array}$ & $\begin{array}{l}\text { Wetting Phase } \\
\text { Saturation (\%) }\end{array}$ & $\begin{array}{c}\text { Incremental } \\
\text { Wetting Phase } \\
\text { Change (\%) }\end{array}$ & $\begin{array}{c}\text { Saturation } \\
\text { Change } \\
\text { per psia }\end{array}$ \\
\hline 41.6 & 5.13 & 0.18 & 100.0 & 0.00 & 0.0000 \\
\hline 45.5 & 4.69 & 0.20 & 100.0 & 0.00 & 0.0000 \\
\hline 49.8 & 4.28 & 0.20 & 100.0 & 0.00 & 0.0000 \\
\hline 54.5 & 3.91 & 0.20 & 100.0 & 0.00 & 0.0000 \\
\hline 59.6 & 3.58 & 0.20 & 100.0 & 0.00 & 0.0000 \\
\hline 65.2 & 3.27 & 0.22 & 100.0 & 0.00 & 0.0000 \\
\hline 71.3 & 2.99 & 0.22 & 100.0 & 0.00 & 0.0000 \\
\hline 78.0 & 2.73 & 0.22 & 100.0 & 0.00 & 0.0000 \\
\hline 85.3 & 2.50 & 0.22 & 100.0 & 0.00 & 0.0000 \\
\hline 93.4 & 2.28 & 0.22 & 100.0 & 0.00 & 0.0000 \\
\hline 102 & 2.09 & 0.24 & 100.0 & 0.00 & 0.0000 \\
\hline 112 & 1.90 & 0.24 & 100.0 & 0.00 & 0.0000 \\
\hline 122 & 1.75 & 0.26 & 100.0 & 0.00 & 0.0000 \\
\hline 134 & 1.59 & 0.26 & 100.0 & 0.00 & 0.0000 \\
\hline 146 & 1.46 & 0.26 & 100.0 & 0.00 & 0.0000 \\
\hline 160 & 1.33 & 0.28 & 100.0 & 0.00 & 0.0000 \\
\hline 175 & 1.22 & 0.30 & 100.0 & 0.00 & 0.0000 \\
\hline 191 & 1.12 & 0.34 & 100.0 & 0.00 & 0.0000 \\
\hline 209 & 1.02 & 0.38 & 100.0 & 0.00 & 0.0000 \\
\hline 229 & 0.932 & 0.42 & 100.0 & 0.00 & 0.0000 \\
\hline 251 & 0.850 & 0.50 & 99.7 & 0.33 & 0.0151 \\
\hline 274 & 0.779 & 0.66 & 99.0 & 0.66 & 0.0289 \\
\hline 300 & 0.711 & 0.81 & 98.4 & 0.58 & 0.0224 \\
\hline 328 & 0.650 & 1.03 & 97.5 & 0.91 & 0.0326 \\
\hline 359 & 0.594 & 1.37 & 96.1 & 1.41 & 0.0455 \\
\hline 393 & 0.543 & 1.79 & 94.4 & 1.74 & 0.0513 \\
\hline 430 & 0.496 & 2.27 & 92.4 & 1.99 & 0.0539 \\
\hline 470 & 0.454 & 2.84 & 90.0 & 2.33 & 0.0581 \\
\hline 514 & 0.415 & 3.46 & 87.5 & 2.57 & 0.0585 \\
\hline 563 & 0.379 & 4.19 & 84.5 & 2.99 & 0.0610 \\
\hline 615 & 0.347 & 4.95 & 81.3 & 3.16 & 0.0607 \\
\hline 673 & 0.317 & 5.56 & 78.8 & 2.49 & 0.0430 \\
\hline 736 & 0.290 & 6.28 & 75.8 & 2.99 & 0.0475 \\
\hline 806 & 0.265 & 6.94 & 73.1 & 2.74 & 0.0392 \\
\hline 881 & 0.242 & 7.69 & 70.0 & 3.07 & 0.0410 \\
\hline 964 & 0.221 & 8.51 & 66.6 & 3.41 & 0.0410 \\
\hline 1050 & 0.203 & 9.34 & 63.2 & 3.41 & 0.0396 \\
\hline 1150 & 0.186 & 10.27 & 59.4 & 3.82 & 0.0382 \\
\hline 1260 & 0.169 & 11.21 & 55.5 & 3.90 & 0.0355 \\
\hline 1380 & 0.155 & 12.04 & 52.1 & 3.41 & 0.0284 \\
\hline 1510 & 0.141 & 12.76 & 49.1 & 2.99 & 0.0230 \\
\hline 1650 & 0.129 & 13.41 & 46.4 & 2.66 & 0.0190 \\
\hline
\end{tabular}


Table 13 (cont.). MICP data for sample 07DL002 - 17.2a.

\section{Mercury Injection Capillary Pressure 07DL002 - 17.2a}

\begin{tabular}{|c|c|c|c|c|c|}
\hline $\begin{array}{c}\text { Capillary } \\
\text { Pressure } \\
\text { (psia) } \\
\end{array}$ & $\begin{array}{l}\text { Pore Aperture } \\
\text { Diameter } \\
\text { (microns) } \\
\end{array}$ & $\begin{array}{l}\text { Cumulative } \\
\text { Bulk Vol. (\%) }\end{array}$ & $\begin{array}{l}\text { Wetting Phase } \\
\text { Saturation (\%) }\end{array}$ & $\begin{array}{c}\text { Incremental } \\
\text { Wetting Phase } \\
\text { Change (\%) } \\
\end{array}$ & $\begin{array}{c}\text { Saturation } \\
\text { Change } \\
\text { per psia }\end{array}$ \\
\hline 1810 & 0.1179 & 14.05 & 43.8 & 2.66 & 0.0166 \\
\hline 1980 & 0.1077 & 14.65 & 41.3 & 2.49 & 0.0147 \\
\hline 2160 & 0.0988 & 15.20 & 39.0 & 2.24 & 0.0125 \\
\hline 2370 & 0.0900 & 15.76 & 36.7 & 2.33 & 0.0111 \\
\hline 2590 & 0.0824 & 16.26 & 34.6 & 2.08 & 0.0094 \\
\hline 2830 & 0.0754 & 16.73 & 32.7 & 1.91 & 0.0080 \\
\hline 3100 & 0.0688 & 17.19 & 30.8 & 1.91 & 0.0071 \\
\hline 3390 & 0.0629 & 17.61 & 29.1 & 1.74 & 0.0060 \\
\hline 3710 & 0.0575 & 17.99 & 27.5 & 1.58 & 0.0049 \\
\hline 4060 & 0.0525 & 18.38 & 25.9 & 1.58 & 0.0045 \\
\hline 4440 & 0.0480 & 18.72 & 24.5 & 1.41 & 0.0037 \\
\hline 4850 & 0.0440 & 19.00 & 23.3 & 1.16 & 0.0028 \\
\hline 5310 & 0.0402 & 19.30 & 22.1 & 1.25 & 0.0027 \\
\hline 5810 & 0.0367 & 19.60 & 20.8 & 1.25 & 0.0025 \\
\hline 6360 & 0.0335 & 19.87 & 19.8 & 1.08 & 0.0020 \\
\hline 6950 & 0.0307 & 20.13 & 18.7 & 1.08 & 0.0018 \\
\hline 7610 & 0.0280 & 20.39 & 17.6 & 1.08 & 0.0016 \\
\hline 8320 & 0.0256 & 20.63 & 16.6 & 1.00 & 0.0014 \\
\hline 9100 & 0.0234 & 20.87 & 15.6 & 1.00 & 0.0013 \\
\hline 9960 & 0.0214 & 21.11 & 14.6 & 1.00 & 0.0012 \\
\hline 10900 & 0.0196 & 21.36 & 13.6 & 1.00 & 0.0011 \\
\hline 11900 & 0.0179 & 21.60 & 12.6 & 1.00 & 0.0010 \\
\hline 13000 & 0.0164 & 21.82 & 11.7 & 0.91 & 0.0008 \\
\hline 14300 & 0.0149 & 22.08 & 10.6 & 1.08 & 0.0008 \\
\hline 15600 & 0.0137 & 22.30 & 9.7 & 0.91 & 0.0007 \\
\hline 17100 & 0.0125 & 22.52 & 8.8 & 0.91 & 0.0006 \\
\hline 18700 & 0.0114 & 22.74 & 7.9 & 0.91 & 0.0006 \\
\hline 20400 & 0.0105 & 22.95 & 7.1 & 0.83 & 0.0005 \\
\hline 22300 & 0.0096 & 23.13 & 6.3 & 0.75 & 0.0004 \\
\hline 24400 & 0.0087 & 23.31 & 5.6 & 0.75 & 0.0004 \\
\hline 26700 & 0.0080 & 23.49 & 4.8 & 0.75 & 0.0003 \\
\hline 29300 & 0.0073 & 23.65 & 4.2 & 0.66 & 0.0003 \\
\hline 32000 & 0.0067 & 23.81 & 3.5 & 0.66 & 0.0002 \\
\hline 35000 & 0.0061 & 23.95 & 2.9 & 0.58 & 0.0002 \\
\hline 38300 & 0.0056 & 24.07 & 2.4 & 0.50 & 0.0002 \\
\hline 41900 & 0.0051 & 24.19 & 1.9 & 0.50 & 0.0001 \\
\hline 45800 & 0.0047 & 24.31 & 1.4 & 0.50 & 0.0001 \\
\hline 50100 & 0.0043 & 24.44 & 0.9 & 0.50 & 0.0001 \\
\hline 54800 & 0.0039 & 24.56 & 0.4 & 0.50 & 0.0001 \\
\hline 59500 & 0.0036 & 24.66 & 0.0 & 0.42 & 0.0001 \\
\hline
\end{tabular}


Table 14. MICP data for sample 07DL002 - 22.0a.

\section{Mercury Injection Capillary Pressure 07DL002 - 22.0a}

\begin{tabular}{|c|c|c|c|c|c|}
\hline \multicolumn{3}{|c|}{\begin{tabular}{ll}
\multicolumn{2}{l}{ Sample Information } \\
\cline { 1 - 2 } Bulk Volume $=$ & $5.0487 \mathrm{cc}$ \\
Pore Volume $=$ & $1.2628 \mathrm{cc}$ \\
Closure $=0.71 \% B V @ 112$ psia
\end{tabular}} & \multicolumn{3}{|c|}{$\begin{array}{l}\text { Porosity }=\quad 25.0 \% \text { (mercury) } \\
\text { Permeability }=\quad 0.171 \text { md (mercury) } \\
\text { Median Pore Aperture }=0.1238 \text { microns (diameter) }\end{array}$} \\
\hline $\begin{array}{l}\text { Capillary } \\
\text { Pressure } \\
\text { (psia) } \\
\end{array}$ & $\begin{array}{l}\text { Pore Aperture } \\
\text { Diameter } \\
\text { (microns) }\end{array}$ & $\begin{array}{c}\text { Cumulative } \\
\text { Bulk Vol. (\%) }\end{array}$ & $\begin{array}{l}\text { Wetting Phase } \\
\text { Saturation (\%) }\end{array}$ & $\begin{array}{l}\text { Incremental } \\
\text { Wetting Phase } \\
\text { Change (\%) } \\
\end{array}$ & $\begin{array}{l}\text { Saturation } \\
\text { Change } \\
\text { per psia }\end{array}$ \\
\hline 1.64 & 130 & 0.02 & 100.0 & 0.00 & 0.0000 \\
\hline 1.80 & 119 & 0.02 & 100.0 & 0.00 & 0.0000 \\
\hline 1.96 & 109 & 0.04 & 100.0 & 0.00 & 0.0000 \\
\hline 2.15 & 99.2 & 0.04 & 100.0 & 0.00 & 0.0000 \\
\hline 2.35 & 90.8 & 0.06 & 100.0 & 0.00 & 0.0000 \\
\hline 2.57 & 83.0 & 0.08 & 100.0 & 0.00 & 0.0000 \\
\hline 2.81 & 75.9 & 0.08 & 100.0 & 0.00 & 0.0000 \\
\hline 3.08 & 69.3 & 0.10 & 100.0 & 0.00 & 0.0000 \\
\hline 3.37 & 63.3 & 0.10 & 100.0 & 0.00 & 0.0000 \\
\hline 3.68 & 58.0 & 0.12 & 100.0 & 0.00 & 0.0000 \\
\hline 4.03 & 52.9 & 0.12 & 100.0 & 0.00 & 0.0000 \\
\hline 4.41 & 48.4 & 0.14 & 100.0 & 0.00 & 0.0000 \\
\hline 4.82 & 44.3 & 0.14 & 100.0 & 0.00 & 0.0000 \\
\hline 5.27 & 40.5 & 0.16 & 100.0 & 0.00 & 0.0000 \\
\hline 5.77 & 37.0 & 0.16 & 100.0 & 0.00 & 0.0000 \\
\hline 6.31 & 33.8 & 0.18 & 100.0 & 0.00 & 0.0000 \\
\hline 6.90 & 30.9 & 0.18 & 100.0 & 0.00 & 0.0000 \\
\hline 7.55 & 28.3 & 0.20 & 100.0 & 0.00 & 0.0000 \\
\hline 8.26 & 25.8 & 0.20 & 100.0 & 0.00 & 0.0000 \\
\hline 9.04 & 23.6 & 0.20 & 100.0 & 0.00 & 0.0000 \\
\hline 9.89 & 21.6 & 0.22 & 100.0 & 0.00 & 0.0000 \\
\hline 10.8 & 19.8 & 0.22 & 100.0 & 0.00 & 0.0000 \\
\hline 11.8 & 18.1 & 0.24 & 100.0 & 0.00 & 0.0000 \\
\hline 12.9 & 16.5 & 0.24 & 100.0 & 0.00 & 0.0000 \\
\hline 14.2 & 15.0 & 0.26 & 100.0 & 0.00 & 0.0000 \\
\hline 15.5 & 13.8 & 0.28 & 100.0 & 0.00 & 0.0000 \\
\hline 16.9 & 12.6 & 0.30 & 100.0 & 0.00 & 0.0000 \\
\hline 18.5 & 11.5 & 0.30 & 100.0 & 0.00 & 0.0000 \\
\hline 20.3 & 10.5 & 0.32 & 100.0 & 0.00 & 0.0000 \\
\hline 22.2 & 9.61 & 0.32 & 100.0 & 0.00 & 0.0000 \\
\hline 24.3 & 8.78 & 0.34 & 100.0 & 0.00 & 0.0000 \\
\hline 26.6 & 8.02 & 0.36 & 100.0 & 0.00 & 0.0000 \\
\hline 29.0 & 7.36 & 0.38 & 100.0 & 0.00 & 0.0000 \\
\hline 31.8 & 6.71 & 0.40 & 100.0 & 0.00 & 0.0000 \\
\hline 34.8 & 6.13 & 0.42 & 100.0 & 0.00 & 0.0000 \\
\hline 38.0 & 5.61 & 0.42 & 100.0 & 0.00 & 0.0000 \\
\hline
\end{tabular}


Table 14 (cont.). MICP data for sample 07DL002 - 22.0a.

\section{Mercury Injection Capillary Pressure 07DL002 - 22.0a}

\begin{tabular}{|c|c|c|c|c|c|}
\hline $\begin{array}{c}\text { Capillary } \\
\text { Pressure } \\
\text { (psia) } \\
\end{array}$ & $\begin{array}{c}\text { Pore Aperture } \\
\text { Diameter } \\
\text { (microns) } \\
\end{array}$ & $\begin{array}{l}\text { Cumulative } \\
\text { Bulk Vol. (\%) } \\
\end{array}$ & $\begin{array}{l}\text { Wetting Phase } \\
\text { Saturation (\%) }\end{array}$ & $\begin{array}{c}\text { Incremental } \\
\text { Wetting Phase } \\
\text { Change (\%) } \\
\end{array}$ & $\begin{array}{c}\text { Saturation } \\
\text { Change } \\
\text { per psia } \\
\end{array}$ \\
\hline 41.6 & 5.13 & 0.46 & 100.0 & 0.00 & 0.0000 \\
\hline 45.5 & 4.69 & 0.48 & 100.0 & 0.00 & 0.0000 \\
\hline 49.8 & 4.28 & 0.48 & 100.0 & 0.00 & 0.0000 \\
\hline 54.5 & 3.91 & 0.50 & 100.0 & 0.00 & 0.0000 \\
\hline 59.6 & 3.58 & 0.50 & 100.0 & 0.00 & 0.0000 \\
\hline 65.2 & 3.27 & 0.52 & 100.0 & 0.00 & 0.0000 \\
\hline 71.3 & 2.99 & 0.54 & 100.0 & 0.00 & 0.0000 \\
\hline 78.0 & 2.73 & 0.54 & 100.0 & 0.00 & 0.0000 \\
\hline 85.3 & 2.50 & 0.55 & 100.0 & 0.00 & 0.0000 \\
\hline 93.4 & 2.28 & 0.59 & 100.0 & 0.00 & 0.0000 \\
\hline 102 & 2.09 & 0.63 & 100.0 & 0.00 & 0.0000 \\
\hline 112 & 1.90 & 0.71 & 100.0 & 0.00 & 0.0000 \\
\hline 122 & 1.75 & 0.79 & 99.7 & 0.32 & 0.0317 \\
\hline 134 & 1.59 & 0.91 & 99.2 & 0.48 & 0.0397 \\
\hline 146 & 1.46 & 1.09 & 98.5 & 0.71 & 0.0595 \\
\hline 160 & 1.33 & 1.33 & 97.5 & 0.95 & 0.0680 \\
\hline 175 & 1.22 & 1.57 & 96.6 & 0.95 & 0.0634 \\
\hline 191 & 1.12 & 1.78 & 95.7 & 0.87 & 0.0545 \\
\hline 209 & 1.02 & 2.00 & 94.8 & 0.87 & 0.0485 \\
\hline 229 & 0.932 & 2.26 & 93.8 & 1.03 & 0.0515 \\
\hline 251 & 0.850 & 2.54 & 92.7 & 1.11 & 0.0505 \\
\hline 274 & 0.779 & 2.79 & 91.7 & 1.03 & 0.0448 \\
\hline 300 & 0.711 & 3.01 & 90.8 & 0.87 & 0.0336 \\
\hline 328 & 0.650 & 3.27 & 89.8 & 1.03 & 0.0368 \\
\hline 359 & 0.594 & 3.55 & 88.7 & 1.11 & 0.0358 \\
\hline 393 & 0.543 & 3.81 & 87.6 & 1.03 & 0.0303 \\
\hline 430 & 0.496 & 4.08 & 86.5 & 1.11 & 0.0300 \\
\hline 470 & 0.454 & 4.36 & 85.4 & 1.11 & 0.0278 \\
\hline 514 & 0.415 & 4.70 & 84.1 & 1.35 & 0.0306 \\
\hline 563 & 0.379 & 4.99 & 82.9 & 1.19 & 0.0243 \\
\hline 615 & 0.347 & 5.37 & 81.4 & 1.51 & 0.0290 \\
\hline 673 & 0.317 & 5.81 & 79.6 & 1.74 & 0.0301 \\
\hline 736 & 0.290 & 6.32 & 77.6 & 2.06 & 0.0327 \\
\hline 806 & 0.265 & 7.02 & 74.8 & 2.78 & 0.0397 \\
\hline 881 & 0.242 & 7.79 & 71.7 & 3.09 & 0.0412 \\
\hline 964 & 0.221 & 8.50 & 68.8 & 2.85 & 0.0344 \\
\hline 1050 & 0.203 & 9.24 & 65.9 & 2.93 & 0.0341 \\
\hline 1150 & 0.186 & 10.05 & 62.6 & 3.25 & 0.0325 \\
\hline 1260 & 0.169 & 10.82 & 59.6 & 3.09 & 0.0281 \\
\hline 1380 & 0.155 & 11.53 & 56.7 & 2.85 & 0.0238 \\
\hline 1510 & 0.141 & 12.21 & 54.0 & 2.70 & 0.0207 \\
\hline 1650 & 0.129 & 12.88 & 51.3 & 2.70 & 0.0193 \\
\hline
\end{tabular}


Table 14 (cont.). MICP data for sample 07DL002 - 22.0a.

\section{Mercury Injection Capillary Pressure 07DL002 - 22.0a}

\begin{tabular}{|c|c|c|c|c|c|}
\hline $\begin{array}{c}\text { Capillary } \\
\text { Pressure } \\
\text { (psia) } \\
\end{array}$ & $\begin{array}{c}\text { Pore Aperture } \\
\text { Diameter } \\
\text { (microns) }\end{array}$ & $\begin{array}{c}\text { Cumulative } \\
\text { Bulk Vol. (\%) } \\
\end{array}$ & $\begin{array}{l}\text { Wetting Phase } \\
\text { Saturation (\%) }\end{array}$ & $\begin{array}{c}\text { Incremental } \\
\text { Wetting Phase } \\
\text { Change (\%) }\end{array}$ & $\begin{array}{c}\text { Saturation } \\
\text { Change } \\
\text { per psia } \\
\end{array}$ \\
\hline 1810 & 0.1179 & 13.56 & 48.6 & 2.70 & 0.0169 \\
\hline 1980 & 0.1077 & 14.21 & 46.0 & 2.62 & 0.0154 \\
\hline 2160 & 0.0988 & 14.82 & 43.5 & 2.46 & 0.0137 \\
\hline 2370 & 0.0900 & 15.48 & 40.9 & 2.62 & 0.0125 \\
\hline 2590 & 0.0824 & 16.09 & 38.5 & 2.46 & 0.0112 \\
\hline 2830 & 0.0754 & 16.67 & 36.2 & 2.30 & 0.0096 \\
\hline 3100 & 0.0688 & 17.24 & 33.9 & 2.30 & 0.0085 \\
\hline 3390 & 0.0629 & 17.76 & 31.8 & 2.06 & 0.0071 \\
\hline 3710 & 0.0575 & 18.25 & 29.8 & 1.98 & 0.0062 \\
\hline 4060 & 0.0525 & 18.71 & 28.0 & 1.82 & 0.0052 \\
\hline 4440 & 0.0480 & 19.13 & 26.3 & 1.67 & 0.0044 \\
\hline 4850 & 0.0440 & 19.46 & 25.0 & 1.35 & 0.0033 \\
\hline 5310 & 0.0402 & 19.82 & 23.6 & 1.43 & 0.0031 \\
\hline 5810 & 0.0367 & 20.16 & 22.2 & 1.35 & 0.0027 \\
\hline 6360 & 0.0335 & 20.47 & 20.9 & 1.27 & 0.0023 \\
\hline 6950 & 0.0307 & 20.75 & 19.8 & 1.11 & 0.0019 \\
\hline 7610 & 0.0280 & 21.03 & 18.7 & 1.11 & 0.0017 \\
\hline 8320 & 0.0256 & 21.31 & 17.6 & 1.11 & 0.0016 \\
\hline 9100 & 0.0234 & 21.56 & 16.6 & 1.03 & 0.0013 \\
\hline 9960 & 0.0214 & 21.82 & 15.5 & 1.03 & 0.0012 \\
\hline 10900 & 0.0196 & 22.08 & 14.5 & 1.03 & 0.0011 \\
\hline 11900 & 0.0179 & 22.32 & 13.6 & 0.95 & 0.0010 \\
\hline 13000 & 0.0164 & 22.55 & 12.6 & 0.95 & 0.0009 \\
\hline 14300 & 0.0149 & 22.81 & 11.6 & 1.03 & 0.0008 \\
\hline 15600 & 0.0137 & 23.05 & 10.6 & 0.95 & 0.0007 \\
\hline 17100 & 0.0125 & 23.29 & 9.7 & 0.95 & 0.0006 \\
\hline 18700 & 0.0114 & 23.53 & 8.7 & 0.95 & 0.0006 \\
\hline 20400 & 0.0105 & 23.72 & 7.9 & 0.79 & 0.0005 \\
\hline 22300 & 0.0096 & 23.94 & 7.1 & 0.87 & 0.0005 \\
\hline 24400 & 0.0087 & 24.14 & 6.3 & 0.79 & 0.0004 \\
\hline 26700 & 0.0080 & 24.34 & 5.5 & 0.79 & 0.0003 \\
\hline 29300 & 0.0073 & 24.52 & 4.8 & 0.71 & 0.0003 \\
\hline 32000 & 0.0067 & 24.67 & 4.1 & 0.63 & 0.0002 \\
\hline 35000 & 0.0061 & 24.83 & 3.5 & 0.63 & 0.0002 \\
\hline 38300 & 0.0056 & 24.99 & 2.9 & 0.63 & 0.0002 \\
\hline 41900 & 0.0051 & 25.13 & 2.3 & 0.56 & 0.0002 \\
\hline 45800 & 0.0047 & 25.27 & 1.7 & 0.56 & 0.0001 \\
\hline 50100 & 0.0043 & 25.39 & 1.3 & 0.48 & 0.0001 \\
\hline 54800 & 0.0039 & 25.53 & 0.7 & 0.56 & 0.0001 \\
\hline 59500 & 0.0036 & 25.71 & 0.0 & 0.71 & 0.0002 \\
\hline
\end{tabular}


Table 15. MICP data for sample 07MAW04 - 0.6.

\section{Mercury Injection Capillary Pressure 07MAW04 - 0.6}

\begin{tabular}{|c|c|c|c|c|c|}
\hline \multicolumn{3}{|c|}{ 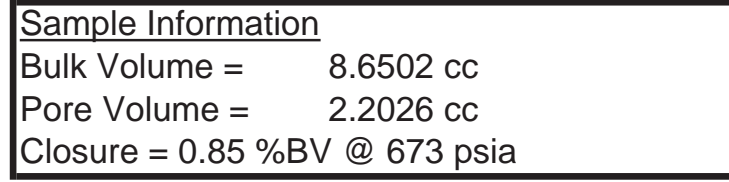 } & \multicolumn{3}{|c|}{$\begin{array}{lc}\text { Porosity }= & 26.6 \% \text { (mercury) } \\
\text { Permeability }= & 0.134 \text { md (mercury) } \\
\text { Median Pore Aperture }=0.1312 \text { microns (diameter) }\end{array}$} \\
\hline $\begin{array}{l}\text { Capillary } \\
\text { Pressure } \\
\text { (psia) } \\
\end{array}$ & $\begin{array}{l}\text { Pore Aperture } \\
\text { Diameter } \\
\text { (microns) } \\
\end{array}$ & $\begin{array}{l}\text { Cumulative } \\
\text { Bulk Vol. (\%) } \\
\end{array}$ & $\begin{array}{l}\text { Wetting Phase } \\
\text { Saturation (\%) } \\
\end{array}$ & $\begin{array}{l}\text { Incremental } \\
\text { Wetting Phase } \\
\text { Change (\%) } \\
\end{array}$ & $\begin{array}{l}\text { Saturation } \\
\text { Change } \\
\text { per psia } \\
\end{array}$ \\
\hline 1.64 & 130 & 0.00 & 100.0 & 0.00 & 0.0000 \\
\hline 1.80 & 119 & 0.02 & 100.0 & 0.00 & 0.0000 \\
\hline 1.96 & 109 & 0.02 & 100.0 & 0.00 & 0.0000 \\
\hline 2.15 & 99.2 & 0.02 & 100.0 & 0.00 & 0.0000 \\
\hline 2.35 & 90.8 & 0.02 & 100.0 & 0.00 & 0.0000 \\
\hline 2.57 & 83.0 & 0.04 & 100.0 & 0.00 & 0.0000 \\
\hline 2.81 & 75.9 & 0.04 & 100.0 & 0.00 & 0.0000 \\
\hline 3.08 & 69.3 & 0.04 & 100.0 & 0.00 & 0.0000 \\
\hline 3.37 & 63.3 & 0.04 & 100.0 & 0.00 & 0.0000 \\
\hline 3.68 & 58.0 & 0.06 & 100.0 & 0.00 & 0.0000 \\
\hline 4.03 & 52.9 & 0.06 & 100.0 & 0.00 & 0.0000 \\
\hline 4.41 & 48.4 & 0.06 & 100.0 & 0.00 & 0.0000 \\
\hline 4.82 & 44.3 & 0.06 & 100.0 & 0.00 & 0.0000 \\
\hline 5.27 & 40.5 & 0.06 & 100.0 & 0.00 & 0.0000 \\
\hline 5.77 & 37.0 & 0.06 & 100.0 & 0.00 & 0.0000 \\
\hline 6.31 & 33.8 & 0.06 & 100.0 & 0.00 & 0.0000 \\
\hline 6.90 & 30.9 & 0.06 & 100.0 & 0.00 & 0.0000 \\
\hline 7.55 & 28.3 & 0.06 & 100.0 & 0.00 & 0.0000 \\
\hline 8.26 & 25.8 & 0.08 & 100.0 & 0.00 & 0.0000 \\
\hline 9.04 & 23.6 & 0.08 & 100.0 & 0.00 & 0.0000 \\
\hline 9.89 & 21.6 & 0.08 & 100.0 & 0.00 & 0.0000 \\
\hline 10.8 & 19.8 & 0.08 & 100.0 & 0.00 & 0.0000 \\
\hline 11.8 & 18.1 & 0.08 & 100.0 & 0.00 & 0.0000 \\
\hline 12.9 & 16.5 & 0.08 & 100.0 & 0.00 & 0.0000 \\
\hline 14.2 & 15.0 & 0.08 & 100.0 & 0.00 & 0.0000 \\
\hline 15.5 & 13.8 & 0.10 & 100.0 & 0.00 & 0.0000 \\
\hline 16.9 & 12.6 & 0.10 & 100.0 & 0.00 & 0.0000 \\
\hline 18.5 & 11.5 & 0.10 & 100.0 & 0.00 & 0.0000 \\
\hline 20.3 & 10.5 & 0.12 & 100.0 & 0.00 & 0.0000 \\
\hline 22.2 & 9.61 & 0.12 & 100.0 & 0.00 & 0.0000 \\
\hline 24.3 & 8.78 & 0.12 & 100.0 & 0.00 & 0.0000 \\
\hline 26.6 & 8.02 & 0.14 & 100.0 & 0.00 & 0.0000 \\
\hline 29.0 & 7.36 & 0.16 & 100.0 & 0.00 & 0.0000 \\
\hline 31.8 & 6.71 & 0.16 & 100.0 & 0.00 & 0.0000 \\
\hline 34.8 & 6.13 & 0.18 & 100.0 & 0.00 & 0.0000 \\
\hline 38.0 & 5.61 & 0.18 & 100.0 & 0.00 & 0.0000 \\
\hline
\end{tabular}


Table 15 (cont.). MICP data for sample 07MAW04 - 0.6.

\section{Mercury Injection Capillary Pressure 07MAW04 - 0.6}

\begin{tabular}{|c|c|c|c|c|c|}
\hline $\begin{array}{c}\text { Capillary } \\
\text { Pressure } \\
\text { (psia) } \\
\end{array}$ & $\begin{array}{c}\text { Pore Aperture } \\
\text { Diameter } \\
\text { (microns) }\end{array}$ & $\begin{array}{c}\text { Cumulative } \\
\text { Bulk Vol. (\%) } \\
\end{array}$ & $\begin{array}{l}\text { Wetting Phase } \\
\text { Saturation (\%) }\end{array}$ & $\begin{array}{c}\text { Incremental } \\
\text { Wetting Phase } \\
\text { Change (\%) } \\
\end{array}$ & $\begin{array}{c}\text { Saturation } \\
\text { Change } \\
\text { per psia } \\
\end{array}$ \\
\hline 41.6 & 5.13 & 0.20 & 100.0 & 0.00 & 0.0000 \\
\hline 45.5 & 4.69 & 0.24 & 100.0 & 0.00 & 0.0000 \\
\hline 49.8 & 4.28 & 0.24 & 100.0 & 0.00 & 0.0000 \\
\hline 54.5 & 3.91 & 0.24 & 100.0 & 0.00 & 0.0000 \\
\hline 59.6 & 3.58 & 0.24 & 100.0 & 0.00 & 0.0000 \\
\hline 65.2 & 3.27 & 0.24 & 100.0 & 0.00 & 0.0000 \\
\hline 71.3 & 2.99 & 0.24 & 100.0 & 0.00 & 0.0000 \\
\hline 78.0 & 2.73 & 0.24 & 100.0 & 0.00 & 0.0000 \\
\hline 85.3 & 2.50 & 0.26 & 100.0 & 0.00 & 0.0000 \\
\hline 93.4 & 2.28 & 0.26 & 100.0 & 0.00 & 0.0000 \\
\hline 102 & 2.09 & 0.26 & 100.0 & 0.00 & 0.0000 \\
\hline 112 & 1.90 & 0.26 & 100.0 & 0.00 & 0.0000 \\
\hline 122 & 1.75 & 0.26 & 100.0 & 0.00 & 0.0000 \\
\hline 134 & 1.59 & 0.26 & 100.0 & 0.00 & 0.0000 \\
\hline 146 & 1.46 & 0.28 & 100.0 & 0.00 & 0.0000 \\
\hline 160 & 1.33 & 0.28 & 100.0 & 0.00 & 0.0000 \\
\hline 175 & 1.22 & 0.28 & 100.0 & 0.00 & 0.0000 \\
\hline 191 & 1.12 & 0.29 & 100.0 & 0.00 & 0.0000 \\
\hline 209 & 1.02 & 0.31 & 100.0 & 0.00 & 0.0000 \\
\hline 229 & 0.932 & 0.33 & 100.0 & 0.00 & 0.0000 \\
\hline 251 & 0.850 & 0.33 & 100.0 & 0.00 & 0.0000 \\
\hline 274 & 0.779 & 0.35 & 100.0 & 0.00 & 0.0000 \\
\hline 300 & 0.711 & 0.37 & 100.0 & 0.00 & 0.0000 \\
\hline 328 & 0.650 & 0.37 & 100.0 & 0.00 & 0.0000 \\
\hline 359 & 0.594 & 0.41 & 100.0 & 0.00 & 0.0000 \\
\hline 393 & 0.543 & 0.43 & 100.0 & 0.00 & 0.0000 \\
\hline 430 & 0.496 & 0.47 & 100.0 & 0.00 & 0.0000 \\
\hline 470 & 0.454 & 0.51 & 100.0 & 0.00 & 0.0000 \\
\hline 514 & 0.415 & 0.55 & 100.0 & 0.00 & 0.0000 \\
\hline 563 & 0.379 & 0.63 & 100.0 & 0.00 & 0.0000 \\
\hline 615 & 0.347 & 0.71 & 100.0 & 0.00 & 0.0000 \\
\hline 673 & 0.317 & 0.85 & 100.0 & 0.00 & 0.0000 \\
\hline 736 & 0.290 & 1.08 & 99.1 & 0.89 & 0.0141 \\
\hline 806 & 0.265 & 1.44 & 97.8 & 1.33 & 0.0190 \\
\hline 881 & 0.242 & 2.06 & 95.4 & 2.36 & 0.0315 \\
\hline 964 & 0.221 & 3.83 & 88.8 & 6.65 & 0.0801 \\
\hline 1050 & 0.203 & 5.88 & 81.1 & 7.68 & 0.0893 \\
\hline 1150 & 0.186 & 8.61 & 70.8 & 10.27 & 0.1027 \\
\hline 1260 & 0.169 & 10.52 & 63.7 & 7.16 & 0.0651 \\
\hline 1380 & 0.155 & 12.05 & 57.9 & 5.76 & 0.0480 \\
\hline 1510 & 0.141 & 13.31 & 53.2 & 4.73 & 0.0364 \\
\hline 1650 & 0.129 & 14.35 & 49.3 & 3.91 & 0.0280 \\
\hline
\end{tabular}


Table 15 (cont.). MICP data for sample 07MAW04 - 0.6.

\section{Mercury Injection Capillary Pressure 07MAW04 - 0.6}

\begin{tabular}{|c|c|c|c|c|c|}
\hline $\begin{array}{c}\text { Capillary } \\
\text { Pressure } \\
\text { (psia) } \\
\end{array}$ & $\begin{array}{c}\text { Pore Aperture } \\
\text { Diameter } \\
\text { (microns) } \\
\end{array}$ & $\begin{array}{c}\text { Cumulative } \\
\text { Bulk Vol. (\%) } \\
\end{array}$ & $\begin{array}{l}\text { Wetting Phase } \\
\text { Saturation (\%) }\end{array}$ & $\begin{array}{c}\text { Incremental } \\
\text { Wetting Phase } \\
\text { Change (\%) } \\
\end{array}$ & $\begin{array}{c}\text { Saturation } \\
\text { Change } \\
\text { per psia } \\
\end{array}$ \\
\hline 1810 & 0.1179 & 15.41 & 45.3 & 3.99 & 0.0249 \\
\hline 1980 & 0.1077 & 16.42 & 41.5 & 3.77 & 0.0222 \\
\hline 2160 & 0.0988 & 17.28 & 38.3 & 3.25 & 0.0181 \\
\hline 2370 & 0.0900 & 18.17 & 34.9 & 3.32 & 0.0158 \\
\hline 2590 & 0.0824 & 18.93 & 32.1 & 2.88 & 0.0131 \\
\hline 2830 & 0.0754 & 19.64 & 29.4 & 2.66 & 0.0111 \\
\hline 3100 & 0.0688 & 20.31 & 26.9 & 2.51 & 0.0093 \\
\hline 3390 & 0.0629 & 20.92 & 24.6 & 2.29 & 0.0079 \\
\hline 3710 & 0.0575 & 21.47 & 22.5 & 2.07 & 0.0065 \\
\hline 4060 & 0.0525 & 21.96 & 20.7 & 1.85 & 0.0053 \\
\hline 4440 & 0.0480 & 22.41 & 19.0 & 1.70 & 0.0045 \\
\hline 4850 & 0.0440 & 22.77 & 17.7 & 1.33 & 0.0032 \\
\hline 5310 & 0.0402 & 23.14 & 16.2 & 1.40 & 0.0031 \\
\hline 5810 & 0.0367 & 23.47 & 15.0 & 1.26 & 0.0025 \\
\hline 6360 & 0.0335 & 23.77 & 13.9 & 1.11 & 0.0020 \\
\hline 6950 & 0.0307 & 24.04 & 12.9 & 1.03 & 0.0018 \\
\hline 7610 & 0.0280 & 24.28 & 12.0 & 0.89 & 0.0013 \\
\hline 8320 & 0.0256 & 24.52 & 11.1 & 0.89 & 0.0012 \\
\hline 9100 & 0.0234 & 24.73 & 10.3 & 0.81 & 0.0010 \\
\hline 9960 & 0.0214 & 24.95 & 9.5 & 0.81 & 0.0009 \\
\hline 10900 & 0.0196 & 25.13 & 8.8 & 0.66 & 0.0007 \\
\hline 11900 & 0.0179 & 25.30 & 8.1 & 0.66 & 0.0007 \\
\hline 13000 & 0.0164 & 25.48 & 7.5 & 0.66 & 0.0006 \\
\hline 14300 & 0.0149 & 25.64 & 6.9 & 0.59 & 0.0005 \\
\hline 15600 & 0.0137 & 25.79 & 6.3 & 0.59 & 0.0005 \\
\hline 17100 & 0.0125 & 25.95 & 5.7 & 0.59 & 0.0004 \\
\hline 18700 & 0.0114 & 26.09 & 5.2 & 0.52 & 0.0003 \\
\hline 20400 & 0.0105 & 26.23 & 4.7 & 0.52 & 0.0003 \\
\hline 22300 & 0.0096 & 26.34 & 4.2 & 0.44 & 0.0002 \\
\hline 24400 & 0.0087 & 26.48 & 3.7 & 0.52 & 0.0002 \\
\hline 26700 & 0.0080 & 26.60 & 3.2 & 0.44 & 0.0002 \\
\hline 29300 & 0.0073 & 26.72 & 2.8 & 0.44 & 0.0002 \\
\hline 32000 & 0.0067 & 26.84 & 2.4 & 0.44 & 0.0002 \\
\hline 35000 & 0.0061 & 26.93 & 2.0 & 0.37 & 0.0001 \\
\hline 38300 & 0.0056 & 27.03 & 1.6 & 0.37 & 0.0001 \\
\hline 41900 & 0.0051 & 27.11 & 1.3 & 0.30 & 0.0001 \\
\hline 45800 & 0.0047 & 27.19 & 1.0 & 0.30 & 0.0001 \\
\hline 50100 & 0.0043 & 27.27 & 0.7 & 0.30 & 0.0001 \\
\hline 54800 & 0.0039 & 27.37 & 0.4 & 0.37 & 0.0001 \\
\hline 59500 & 0.0036 & 27.46 & 0.0 & 0.37 & 0.0001 \\
\hline
\end{tabular}


Table 16. MICP data for sample 07MAW04 - 10.3.

\section{Mercury Injection Capillary Pressure 07MAW04 - 10.3}

\begin{tabular}{|c|c|c|c|c|c|}
\hline \\
\hline \multicolumn{3}{|c|}{$\begin{array}{l}\text { Bulk Volume }=5.7984 \mathrm{cc} \\
\text { Pore Volume }=\quad 1.3388 \mathrm{cc} \\
\text { Closure = 1.18 \%BV @ } 673 \text { psia }\end{array}$} & \multicolumn{3}{|c|}{$\begin{array}{lc}\text { Porosity }= & 23.1 \% \text { (merury) } \\
\text { Permeability }= & 0.0808 \mathrm{md} \text { (mercury) } \\
\text { Median Pore Aperture }=0.1095 \text { microns (diameter) }\end{array}$} \\
\hline $\begin{array}{l}\text { Capillary } \\
\text { Pressure } \\
\text { (psia) } \\
\end{array}$ & $\begin{array}{c}\text { Pore Aperture } \\
\text { Diameter } \\
\text { (microns) } \\
\end{array}$ & $\begin{array}{l}\text { Cumulative } \\
\text { Bulk Vol. (\%) } \\
\end{array}$ & $\begin{array}{l}\text { Wetting Phase } \\
\text { Saturation (\%) }\end{array}$ & $\begin{array}{l}\text { Incremental } \\
\text { Wetting Phase } \\
\text { Change (\%) } \\
\end{array}$ & $\begin{array}{l}\text { Saturation } \\
\text { Change } \\
\text { per psia } \\
\end{array}$ \\
\hline 1.64 & 130 & 0.02 & 100.0 & 0.00 & 0.0000 \\
\hline 1.80 & 119 & 0.02 & 100.0 & 0.00 & 0.0000 \\
\hline 1.96 & 109 & 0.04 & 100.0 & 0.00 & 0.0000 \\
\hline 2.15 & 99.2 & 0.06 & 100.0 & 0.00 & 0.0000 \\
\hline 2.35 & 90.8 & 0.08 & 100.0 & 0.00 & 0.0000 \\
\hline 2.57 & 83.0 & 0.10 & 100.0 & 0.00 & 0.0000 \\
\hline 2.81 & 75.9 & 0.10 & 100.0 & 0.00 & 0.0000 \\
\hline 3.08 & 69.3 & 0.12 & 100.0 & 0.00 & 0.0000 \\
\hline 3.37 & 63.3 & 0.12 & 100.0 & 0.00 & 0.0000 \\
\hline 3.68 & 58.0 & 0.14 & 100.0 & 0.00 & 0.0000 \\
\hline 4.03 & 52.9 & 0.16 & 100.0 & 0.00 & 0.0000 \\
\hline 4.41 & 48.4 & 0.16 & 100.0 & 0.00 & 0.0000 \\
\hline 4.82 & 44.3 & 0.18 & 100.0 & 0.00 & 0.0000 \\
\hline 5.27 & 40.5 & 0.18 & 100.0 & 0.00 & 0.0000 \\
\hline 5.77 & 37.0 & 0.20 & 100.0 & 0.00 & 0.0000 \\
\hline 6.31 & 33.8 & 0.20 & 100.0 & 0.00 & 0.0000 \\
\hline 6.90 & 30.9 & 0.22 & 100.0 & 0.00 & 0.0000 \\
\hline 7.55 & 28.3 & 0.24 & 100.0 & 0.00 & 0.0000 \\
\hline 8.26 & 25.8 & 0.24 & 100.0 & 0.00 & 0.0000 \\
\hline 9.04 & 23.6 & 0.24 & 100.0 & 0.00 & 0.0000 \\
\hline 9.89 & 21.6 & 0.26 & 100.0 & 0.00 & 0.0000 \\
\hline 10.8 & 19.8 & 0.28 & 100.0 & 0.00 & 0.0000 \\
\hline 11.8 & 18.1 & 0.28 & 100.0 & 0.00 & 0.0000 \\
\hline 12.9 & 16.5 & 0.31 & 100.0 & 0.00 & 0.0000 \\
\hline 14.2 & 15.0 & 0.31 & 100.0 & 0.00 & 0.0000 \\
\hline 15.5 & 13.8 & 0.33 & 100.0 & 0.00 & 0.0000 \\
\hline 16.9 & 12.6 & 0.35 & 100.0 & 0.00 & 0.0000 \\
\hline 18.5 & 11.5 & 0.35 & 100.0 & 0.00 & 0.0000 \\
\hline 20.3 & 10.5 & 0.37 & 100.0 & 0.00 & 0.0000 \\
\hline 22.2 & 9.61 & 0.37 & 100.0 & 0.00 & 0.0000 \\
\hline 24.3 & 8.78 & 0.39 & 100.0 & 0.00 & 0.0000 \\
\hline 26.6 & 8.02 & 0.41 & 100.0 & 0.00 & 0.0000 \\
\hline 29.0 & 7.36 & 0.43 & 100.0 & 0.00 & 0.0000 \\
\hline 31.8 & 6.71 & 0.45 & 100.0 & 0.00 & 0.0000 \\
\hline 34.8 & 6.13 & 0.47 & 100.0 & 0.00 & 0.0000 \\
\hline 38.0 & 5.61 & 0.49 & 100.0 & 0.00 & 0.0000 \\
\hline
\end{tabular}


Table 16 (cont.). MICP data for sample 07MAW04 - 10.3.

\section{Mercury Injection Capillary Pressure 07MAW04 - 10.3}

\begin{tabular}{|c|c|c|c|c|c|}
\hline $\begin{array}{c}\text { Capillary } \\
\text { Pressure } \\
\text { (psia) } \\
\end{array}$ & $\begin{array}{c}\text { Pore Aperture } \\
\text { Diameter } \\
\text { (microns) } \\
\end{array}$ & $\begin{array}{c}\text { Cumulative } \\
\text { Bulk Vol. (\%) }\end{array}$ & $\begin{array}{l}\text { Wetting Phase } \\
\text { Saturation (\%) }\end{array}$ & $\begin{array}{c}\text { Incremental } \\
\text { Wetting Phase } \\
\text { Change (\%) } \\
\end{array}$ & $\begin{array}{c}\text { Saturation } \\
\text { Change } \\
\text { per psia }\end{array}$ \\
\hline 41.6 & 5.13 & 0.49 & 100.0 & 0.00 & 0.0000 \\
\hline 45.5 & 4.69 & 0.53 & 100.0 & 0.00 & 0.0000 \\
\hline 49.8 & 4.28 & 0.53 & 100.0 & 0.00 & 0.0000 \\
\hline 54.5 & 3.91 & 0.53 & 100.0 & 0.00 & 0.0000 \\
\hline 59.6 & 3.58 & 0.53 & 100.0 & 0.00 & 0.0000 \\
\hline 65.2 & 3.27 & 0.55 & 100.0 & 0.00 & 0.0000 \\
\hline 71.3 & 2.99 & 0.55 & 100.0 & 0.00 & 0.0000 \\
\hline 78.0 & 2.73 & 0.55 & 100.0 & 0.00 & 0.0000 \\
\hline 85.3 & 2.50 & 0.57 & 100.0 & 0.00 & 0.0000 \\
\hline 93.4 & 2.28 & 0.57 & 100.0 & 0.00 & 0.0000 \\
\hline 102 & 2.09 & 0.59 & 100.0 & 0.00 & 0.0000 \\
\hline 112 & 1.90 & 0.59 & 100.0 & 0.00 & 0.0000 \\
\hline 122 & 1.75 & 0.61 & 100.0 & 0.00 & 0.0000 \\
\hline 134 & 1.59 & 0.63 & 100.0 & 0.00 & 0.0000 \\
\hline 146 & 1.46 & 0.63 & 100.0 & 0.00 & 0.0000 \\
\hline 160 & 1.33 & 0.63 & 100.0 & 0.00 & 0.0000 \\
\hline 175 & 1.22 & 0.65 & 100.0 & 0.00 & 0.0000 \\
\hline 191 & 1.12 & 0.65 & 100.0 & 0.00 & 0.0000 \\
\hline 209 & 1.02 & 0.67 & 100.0 & 0.00 & 0.0000 \\
\hline 229 & 0.932 & 0.69 & 100.0 & 0.00 & 0.0000 \\
\hline 251 & 0.850 & 0.71 & 100.0 & 0.00 & 0.0000 \\
\hline 274 & 0.779 & 0.71 & 100.0 & 0.00 & 0.0000 \\
\hline 300 & 0.711 & 0.73 & 100.0 & 0.00 & 0.0000 \\
\hline 328 & 0.650 & 0.75 & 100.0 & 0.00 & 0.0000 \\
\hline 359 & 0.594 & 0.77 & 100.0 & 0.00 & 0.0000 \\
\hline 393 & 0.543 & 0.81 & 100.0 & 0.00 & 0.0000 \\
\hline 430 & 0.496 & 0.85 & 100.0 & 0.00 & 0.0000 \\
\hline 470 & 0.454 & 0.90 & 100.0 & 0.00 & 0.0000 \\
\hline 514 & 0.415 & 0.94 & 100.0 & 0.00 & 0.0000 \\
\hline 563 & 0.379 & 1.00 & 100.0 & 0.00 & 0.0000 \\
\hline 615 & 0.347 & 1.06 & 100.0 & 0.00 & 0.0000 \\
\hline 673 & 0.317 & 1.18 & 100.0 & 0.00 & 0.0000 \\
\hline 736 & 0.290 & 1.32 & 99.4 & 0.62 & 0.0098 \\
\hline 806 & 0.265 & 1.53 & 98.5 & 0.88 & 0.0126 \\
\hline 881 & 0.242 & 1.83 & 97.2 & 1.32 & 0.0176 \\
\hline 964 & 0.221 & 2.36 & 94.9 & 2.29 & 0.0276 \\
\hline 1050 & 0.203 & 2.99 & 92.2 & 2.73 & 0.0318 \\
\hline 1150 & 0.186 & 4.17 & 87.0 & 5.11 & 0.0511 \\
\hline 1260 & 0.169 & 5.92 & 79.5 & 7.58 & 0.0689 \\
\hline 1380 & 0.155 & 7.71 & 71.7 & 7.76 & 0.0647 \\
\hline 1510 & 0.141 & 9.26 & 65.0 & 6.70 & 0.0516 \\
\hline 1650 & 0.129 & 10.64 & 59.0 & 6.00 & 0.0428 \\
\hline
\end{tabular}


Table 16 (cont.). MICP data for sample 07MAW04 - 10.3.

\section{Mercury Injection Capillary Pressure 07MAW04 - 10.3}

\begin{tabular}{|c|c|c|c|c|c|}
\hline $\begin{array}{c}\text { Capillary } \\
\text { Pressure } \\
\text { (psia) } \\
\end{array}$ & $\begin{array}{c}\text { Pore Aperture } \\
\text { Diameter } \\
\text { (microns) } \\
\end{array}$ & $\begin{array}{c}\text { Cumulative } \\
\text { Bulk Vol. (\%) } \\
\end{array}$ & $\begin{array}{l}\text { Wetting Phase } \\
\text { Saturation (\%) }\end{array}$ & $\begin{array}{c}\text { Incremental } \\
\text { Wetting Phase } \\
\text { Change (\%) } \\
\end{array}$ & $\begin{array}{c}\text { Saturation } \\
\text { Change } \\
\text { per psia } \\
\end{array}$ \\
\hline 1810 & 0.1179 & 11.84 & 53.8 & 5.20 & 0.0325 \\
\hline 1980 & 0.1077 & 12.90 & 49.2 & 4.59 & 0.0270 \\
\hline 2160 & 0.0988 & 13.81 & 45.2 & 3.97 & 0.0220 \\
\hline 2370 & 0.0900 & 14.69 & 41.4 & 3.79 & 0.0181 \\
\hline 2590 & 0.0824 & 15.42 & 38.3 & 3.17 & 0.0144 \\
\hline 2830 & 0.0754 & 16.09 & 35.4 & 2.91 & 0.0121 \\
\hline 3100 & 0.0688 & 16.70 & 32.7 & 2.65 & 0.0098 \\
\hline 3390 & 0.0629 & 17.23 & 30.4 & 2.29 & 0.0079 \\
\hline 3710 & 0.0575 & 17.72 & 28.3 & 2.12 & 0.0066 \\
\hline 4060 & 0.0525 & 18.17 & 26.4 & 1.94 & 0.0055 \\
\hline 4440 & 0.0480 & 18.57 & 24.6 & 1.76 & 0.0046 \\
\hline 4850 & 0.0440 & 18.92 & 23.1 & 1.50 & 0.0037 \\
\hline 5310 & 0.0402 & 19.27 & 21.6 & 1.50 & 0.0033 \\
\hline 5810 & 0.0367 & 19.59 & 20.2 & 1.41 & 0.0028 \\
\hline 6360 & 0.0335 & 19.90 & 18.9 & 1.32 & 0.0024 \\
\hline 6950 & 0.0307 & 20.18 & 17.6 & 1.23 & 0.0021 \\
\hline 7610 & 0.0280 & 20.45 & 16.5 & 1.15 & 0.0017 \\
\hline 8320 & 0.0256 & 20.71 & 15.3 & 1.15 & 0.0016 \\
\hline 9100 & 0.0234 & 20.95 & 14.3 & 1.06 & 0.0014 \\
\hline 9960 & 0.0214 & 21.18 & 13.3 & 0.97 & 0.0011 \\
\hline 10900 & 0.0196 & 21.40 & 12.3 & 0.97 & 0.0010 \\
\hline 11900 & 0.0179 & 21.60 & 11.5 & 0.88 & 0.0009 \\
\hline 13000 & 0.0164 & 21.81 & 10.6 & 0.88 & 0.0008 \\
\hline 14300 & 0.0149 & 22.01 & 9.7 & 0.88 & 0.0007 \\
\hline 15600 & 0.0137 & 22.17 & 9.0 & 0.71 & 0.0005 \\
\hline 17100 & 0.0125 & 22.36 & 8.2 & 0.79 & 0.0005 \\
\hline 18700 & 0.0114 & 22.52 & 7.5 & 0.71 & 0.0004 \\
\hline 20400 & 0.0105 & 22.68 & 6.8 & 0.71 & 0.0004 \\
\hline 22300 & 0.0096 & 22.85 & 6.1 & 0.71 & 0.0004 \\
\hline 24400 & 0.0087 & 22.99 & 5.5 & 0.62 & 0.0003 \\
\hline 26700 & 0.0080 & 23.13 & 4.9 & 0.62 & 0.0003 \\
\hline 29300 & 0.0073 & 23.29 & 4.1 & 0.71 & 0.0003 \\
\hline 32000 & 0.0067 & 23.42 & 3.6 & 0.53 & 0.0002 \\
\hline 35000 & 0.0061 & 23.54 & 3.1 & 0.53 & 0.0002 \\
\hline 38300 & 0.0056 & 23.66 & 2.6 & 0.53 & 0.0002 \\
\hline 41900 & 0.0051 & 23.78 & 2.0 & 0.53 & 0.0001 \\
\hline 45800 & 0.0047 & 23.88 & 1.6 & 0.44 & 0.0001 \\
\hline 50100 & 0.0043 & 23.99 & 1.1 & 0.44 & 0.0001 \\
\hline 54800 & 0.0039 & 24.13 & 0.5 & 0.62 & 0.0001 \\
\hline 59500 & 0.0036 & 24.25 & 0.0 & 0.53 & 0.0001 \\
\hline
\end{tabular}


Table 17. MICP data for sample ST4 - 20.

\section{Mercury Injection Capillary Pressure ST4 - 20}

\begin{tabular}{|c|c|c|c|c|c|}
\hline \multicolumn{3}{|c|}{ Sample Information } & \multirow{2}{*}{\multicolumn{3}{|c|}{$\begin{array}{ll}\text { Porosity }= & 35.7 \% \text { (mercury) } \\
\text { Permeability }= & 0.215 \text { md (mercury) } \\
\text { Median Pore Aperture }=0.1308 \text { microns (diameter) }\end{array}$}} \\
\hline $\begin{array}{l}\text { Bulk Volume }= \\
\text { Pore Volume } \\
\text { Closure }=1.01\end{array}$ & $\begin{array}{r}5.3015 \mathrm{cc} \\
1.8914 \mathrm{cc} \\
\text { oBV @ } 470 \text { psia }\end{array}$ & & & & \\
\hline $\begin{array}{l}\text { Capillary } \\
\text { Pressure } \\
\text { (psia) }\end{array}$ & $\begin{array}{l}\text { Pore Aperture } \\
\text { Diameter } \\
\text { (microns) } \\
\end{array}$ & $\begin{array}{c}\text { Cumulative } \\
\text { Bulk Vol. (\%) } \\
\end{array}$ & $\begin{array}{l}\text { Wetting Phase } \\
\text { Saturation (\%) }\end{array}$ & $\begin{array}{l}\text { Incremental } \\
\text { Wetting Phase } \\
\text { Change (\%) } \\
\end{array}$ & $\begin{array}{l}\text { Saturation } \\
\text { Change } \\
\text { per psia } \\
\end{array}$ \\
\hline 1.64 & 130 & 0.02 & 100.0 & 0.00 & 0.0000 \\
\hline 1.80 & 119 & 0.02 & 100.0 & 0.00 & 0.0000 \\
\hline 1.96 & 109 & 0.03 & 100.0 & 0.00 & 0.0000 \\
\hline 2.15 & 99.2 & 0.03 & 100.0 & 0.00 & 0.0000 \\
\hline 2.35 & 90.8 & 0.05 & 100.0 & 0.00 & 0.0000 \\
\hline 2.57 & 83.0 & 0.05 & 100.0 & 0.00 & 0.0000 \\
\hline 2.81 & 75.9 & 0.05 & 100.0 & 0.00 & 0.0000 \\
\hline 3.08 & 69.3 & 0.07 & 100.0 & 0.00 & 0.0000 \\
\hline 3.37 & 63.3 & 0.07 & 100.0 & 0.00 & 0.0000 \\
\hline 3.68 & 58.0 & 0.07 & 100.0 & 0.00 & 0.0000 \\
\hline 4.03 & 52.9 & 0.09 & 100.0 & 0.00 & 0.0000 \\
\hline 4.41 & 48.4 & 0.09 & 100.0 & 0.00 & 0.0000 \\
\hline 4.82 & 44.3 & 0.09 & 100.0 & 0.00 & 0.0000 \\
\hline 5.27 & 40.5 & 0.09 & 100.0 & 0.00 & 0.0000 \\
\hline 5.77 & 37.0 & 0.09 & 100.0 & 0.00 & 0.0000 \\
\hline 6.31 & 33.8 & 0.09 & 100.0 & 0.00 & 0.0000 \\
\hline 6.90 & 30.9 & 0.09 & 100.0 & 0.00 & 0.0000 \\
\hline 7.55 & 28.3 & 0.09 & 100.0 & 0.00 & 0.0000 \\
\hline 8.26 & 25.8 & 0.09 & 100.0 & 0.00 & 0.0000 \\
\hline 9.04 & 23.6 & 0.09 & 100.0 & 0.00 & 0.0000 \\
\hline 9.89 & 21.6 & 0.09 & 100.0 & 0.00 & 0.0000 \\
\hline 10.8 & 19.8 & 0.09 & 100.0 & 0.00 & 0.0000 \\
\hline 11.8 & 18.1 & 0.09 & 100.0 & 0.00 & 0.0000 \\
\hline 12.9 & 16.5 & 0.09 & 100.0 & 0.00 & 0.0000 \\
\hline 14.2 & 15.0 & 0.09 & 100.0 & 0.00 & 0.0000 \\
\hline 15.5 & 13.8 & 0.09 & 100.0 & 0.00 & 0.0000 \\
\hline 16.9 & 12.6 & 0.09 & 100.0 & 0.00 & 0.0000 \\
\hline 18.5 & 11.5 & 0.09 & 100.0 & 0.00 & 0.0000 \\
\hline 20.3 & 10.5 & 0.09 & 100.0 & 0.00 & 0.0000 \\
\hline 22.2 & 9.61 & 0.20 & 100.0 & 0.00 & 0.0000 \\
\hline 24.3 & 8.78 & 0.20 & 100.0 & 0.00 & 0.0000 \\
\hline 26.6 & 8.02 & 0.22 & 100.0 & 0.00 & 0.0000 \\
\hline 29.0 & 7.36 & 0.22 & 100.0 & 0.00 & 0.0000 \\
\hline 31.8 & 6.71 & 0.24 & 100.0 & 0.00 & 0.0000 \\
\hline 34.8 & 6.13 & 0.26 & 100.0 & 0.00 & 0.0000 \\
\hline 38.0 & 5.61 & 0.26 & 100.0 & 0.00 & 0.0000 \\
\hline
\end{tabular}


Table 17 (cont.). MICP data for sample ST4 - 20.

\section{Mercury Injection Capillary Pressure ST4 - 20}

\begin{tabular}{|c|c|c|c|c|c|}
\hline $\begin{array}{l}\text { Capillary } \\
\text { Pressure } \\
\text { (psia) }\end{array}$ & $\begin{array}{l}\text { Pore Aperture } \\
\text { Diameter } \\
\text { (microns) }\end{array}$ & $\begin{array}{c}\text { Cumulative } \\
\text { Bulk Vol. (\%) }\end{array}$ & $\begin{array}{l}\text { Wetting Phase } \\
\text { Saturation (\%) }\end{array}$ & $\begin{array}{c}\text { Incremental } \\
\text { Wetting Phase } \\
\text { Change (\%) }\end{array}$ & $\begin{array}{c}\text { Saturation } \\
\text { Change } \\
\text { per psia }\end{array}$ \\
\hline 41.6 & 5.13 & 0.27 & 100.0 & 0.00 & 0.0000 \\
\hline 45.5 & 4.69 & 0.29 & 100.0 & 0.00 & 0.0000 \\
\hline 49.8 & 4.28 & 0.29 & 100.0 & 0.00 & 0.0000 \\
\hline 54.5 & 3.91 & 0.29 & 100.0 & 0.00 & 0.0000 \\
\hline 59.6 & 3.58 & 0.29 & 100.0 & 0.00 & 0.0000 \\
\hline 65.2 & 3.27 & 0.29 & 100.0 & 0.00 & 0.0000 \\
\hline 71.3 & 2.99 & 0.29 & 100.0 & 0.00 & 0.0000 \\
\hline 78.0 & 2.73 & 0.31 & 100.0 & 0.00 & 0.0000 \\
\hline 85.3 & 2.50 & 0.31 & 100.0 & 0.00 & 0.0000 \\
\hline 93.4 & 2.28 & 0.31 & 100.0 & 0.00 & 0.0000 \\
\hline 102 & 2.09 & 0.31 & 100.0 & 0.00 & 0.0000 \\
\hline 112 & 1.90 & 0.31 & 100.0 & 0.00 & 0.0000 \\
\hline 122 & 1.75 & 0.32 & 100.0 & 0.00 & 0.0000 \\
\hline 134 & 1.59 & 0.32 & 100.0 & 0.00 & 0.0000 \\
\hline 146 & 1.46 & 0.34 & 100.0 & 0.00 & 0.0000 \\
\hline 160 & 1.33 & 0.36 & 100.0 & 0.00 & 0.0000 \\
\hline 175 & 1.22 & 0.38 & 100.0 & 0.00 & 0.0000 \\
\hline 191 & 1.12 & 0.43 & 100.0 & 0.00 & 0.0000 \\
\hline 209 & 1.02 & 0.44 & 100.0 & 0.00 & 0.0000 \\
\hline 229 & 0.932 & 0.48 & 100.0 & 0.00 & 0.0000 \\
\hline 251 & 0.850 & 0.49 & 100.0 & 0.00 & 0.0000 \\
\hline 274 & 0.779 & 0.53 & 100.0 & 0.00 & 0.0000 \\
\hline 300 & 0.711 & 0.58 & 100.0 & 0.00 & 0.0000 \\
\hline 328 & 0.650 & 0.63 & 100.0 & 0.00 & 0.0000 \\
\hline 359 & 0.594 & 0.68 & 100.0 & 0.00 & 0.0000 \\
\hline 393 & 0.543 & 0.77 & 100.0 & 0.00 & 0.0000 \\
\hline 430 & 0.496 & 0.87 & 100.0 & 0.00 & 0.0000 \\
\hline 470 & 0.454 & 1.01 & 100.0 & 0.00 & 0.0000 \\
\hline 514 & 0.415 & 1.19 & 99.5 & 0.53 & 0.0120 \\
\hline 563 & 0.379 & 1.50 & 98.6 & 0.86 & 0.0176 \\
\hline 615 & 0.347 & 1.88 & 97.6 & 1.05 & 0.0202 \\
\hline 673 & 0.317 & 2.28 & 96.4 & 1.15 & 0.0198 \\
\hline 736 & 0.290 & 2.90 & 94.7 & 1.72 & 0.0273 \\
\hline 806 & 0.265 & 3.31 & 93.5 & 1.15 & 0.0164 \\
\hline 881 & 0.242 & 3.99 & 91.6 & 1.91 & 0.0255 \\
\hline 964 & 0.221 & 4.84 & 89.2 & 2.39 & 0.0288 \\
\hline 1050 & 0.203 & 5.88 & 86.3 & 2.92 & 0.0339 \\
\hline 1150 & 0.186 & 7.47 & 81.9 & 4.45 & 0.0445 \\
\hline 1260 & 0.169 & 10.11 & 74.5 & 7.41 & 0.0674 \\
\hline 1380 & 0.155 & 13.19 & 65.8 & 8.66 & 0.0721 \\
\hline 1510 & 0.141 & 17.03 & 55.0 & 10.76 & 0.0828 \\
\hline 1650 & 0.129 & 19.16 & 49.1 & 5.98 & 0.0427 \\
\hline
\end{tabular}


Table 17 (cont.). MICP data for sample ST4 - 20.

\section{Mercury Injection Capillary Pressure ST4 - 20}

\begin{tabular}{|c|c|c|c|c|c|}
\hline $\begin{array}{c}\text { Capillary } \\
\text { Pressure } \\
\text { (psia) } \\
\end{array}$ & $\begin{array}{l}\text { Pore Aperture } \\
\text { Diameter } \\
\text { (microns) }\end{array}$ & $\begin{array}{c}\text { Cumulative } \\
\text { Bulk Vol. (\%) }\end{array}$ & $\begin{array}{l}\text { Wetting Phase } \\
\text { Saturation (\%) }\end{array}$ & $\begin{array}{c}\text { Incremental } \\
\text { Wetting Phase } \\
\text { Change (\%) }\end{array}$ & $\begin{array}{c}\text { Saturation } \\
\text { Change } \\
\text { per psia } \\
\end{array}$ \\
\hline 1810 & 0.1179 & 21.10 & 43.6 & 5.45 & 0.0341 \\
\hline 1980 & 0.1077 & 22.74 & 39.0 & 4.59 & 0.0270 \\
\hline 2160 & 0.0988 & 24.07 & 35.3 & 3.73 & 0.0207 \\
\hline 2370 & 0.0900 & 25.28 & 31.9 & 3.40 & 0.0162 \\
\hline 2590 & 0.0824 & 26.32 & 29.0 & 2.92 & 0.0133 \\
\hline 2830 & 0.0754 & 27.24 & 26.4 & 2.58 & 0.0108 \\
\hline 3100 & 0.0688 & 28.11 & 24.0 & 2.44 & 0.0090 \\
\hline 3390 & 0.0629 & 28.84 & 21.9 & 2.06 & 0.0071 \\
\hline 3710 & 0.0575 & 29.52 & 20.0 & 1.91 & 0.0060 \\
\hline 4060 & 0.0525 & 30.12 & 18.3 & 1.67 & 0.0048 \\
\hline 4440 & 0.0480 & 30.65 & 16.8 & 1.48 & 0.0039 \\
\hline 4850 & 0.0440 & 31.08 & 15.6 & 1.20 & 0.0029 \\
\hline 5310 & 0.0402 & 31.50 & 14.4 & 1.20 & 0.0026 \\
\hline 5810 & 0.0367 & 31.88 & 13.4 & 1.05 & 0.0021 \\
\hline 6360 & 0.0335 & 32.20 & 12.5 & 0.91 & 0.0017 \\
\hline 6950 & 0.0307 & 32.51 & 11.6 & 0.86 & 0.0015 \\
\hline 7610 & 0.0280 & 32.78 & 10.9 & 0.77 & 0.0012 \\
\hline 8320 & 0.0256 & 33.02 & 10.2 & 0.67 & 0.0009 \\
\hline 9100 & 0.0234 & 33.24 & 9.6 & 0.62 & 0.0008 \\
\hline 9960 & 0.0214 & 33.44 & 9.0 & 0.57 & 0.0007 \\
\hline 10900 & 0.0196 & 33.65 & 8.4 & 0.57 & 0.0006 \\
\hline 11900 & 0.0179 & 33.84 & 7.9 & 0.53 & 0.0005 \\
\hline 13000 & 0.0164 & 34.02 & 7.4 & 0.53 & 0.0005 \\
\hline 14300 & 0.0149 & 34.21 & 6.8 & 0.53 & 0.0004 \\
\hline 15600 & 0.0137 & 34.38 & 6.4 & 0.48 & 0.0004 \\
\hline 17100 & 0.0125 & 34.55 & 5.9 & 0.48 & 0.0003 \\
\hline 18700 & 0.0114 & 34.72 & 5.4 & 0.48 & 0.0003 \\
\hline 20400 & 0.0105 & 34.88 & 5.0 & 0.43 & 0.0003 \\
\hline 22300 & 0.0096 & 35.03 & 4.5 & 0.43 & 0.0002 \\
\hline 24400 & 0.0087 & 35.20 & 4.1 & 0.48 & 0.0002 \\
\hline 26700 & 0.0080 & 35.35 & 3.6 & 0.43 & 0.0002 \\
\hline 29300 & 0.0073 & 35.51 & 3.2 & 0.43 & 0.0002 \\
\hline 32000 & 0.0067 & 35.64 & 2.8 & 0.38 & 0.0001 \\
\hline 35000 & 0.0061 & 35.80 & 2.4 & 0.43 & 0.0001 \\
\hline 38300 & 0.0056 & 35.93 & 2.0 & 0.38 & 0.0001 \\
\hline 41900 & 0.0051 & 36.05 & 1.7 & 0.33 & 0.0001 \\
\hline 45800 & 0.0047 & 36.19 & 1.3 & 0.38 & 0.0001 \\
\hline 50100 & 0.0043 & 36.34 & 0.9 & 0.43 & 0.0001 \\
\hline 54800 & 0.0039 & 36.51 & 0.4 & 0.48 & 0.0001 \\
\hline 59500 & 0.0036 & 36.65 & 0.0 & 0.38 & 0.0001 \\
\hline
\end{tabular}

